\title{
Characteristics of a folate receptor- $\alpha$ GPI-anchored into a multilipid bilayer obtained from atomistic molecular dynamics simulations
}

Gergana Gocheva, ${ }^{a}$ Nikoleta Ivanova, ${ }^{\mathrm{a}, \mathrm{b}}$ Stoyan Iliev, ${ }^{\mathrm{a}}$ Jasmina Petrova, ${ }^{\mathrm{a}}$ Galia Madjarova, ${ }^{\mathrm{a}}$ Anela Ivanova ${ }^{\mathrm{a}, *}$

aSofia University "St. Kliment Ohridski”, Faculty of Chemistry and Pharmacy, Laboratory of Quantum and Computational Chemistry, 1 James Bourchier blvd., 1164 Sofia, Bulgaria *Corresponding author: E-mail: aivanova@chem.uni-sofia.bg; Tel.: ++35928161520; FAX: $++35929625438$

\section{Supporting information}

\footnotetext{
${ }^{\mathrm{b}}$ Current address: Department of Physical Chemistry, University of Chemical Technology and Metallurgy,
} 8 Kliment Ohridski blvd., 1756 Sofia, Bulgaria 


\section{Modified lipids}

Some of the membrane lipids, which were registered experimentally [Refs. 36, 37 of the main manuscript], are not present in the CHARMM-GUI interface, which is used to construct the initial configuration of the lipid bilayer. Therefore, similar 'standard' molecules are placed instead of them, which are subsequently manually modified to the appropriate chemical structure. The particular modifications are shown in Scheme S1 below.

POPC

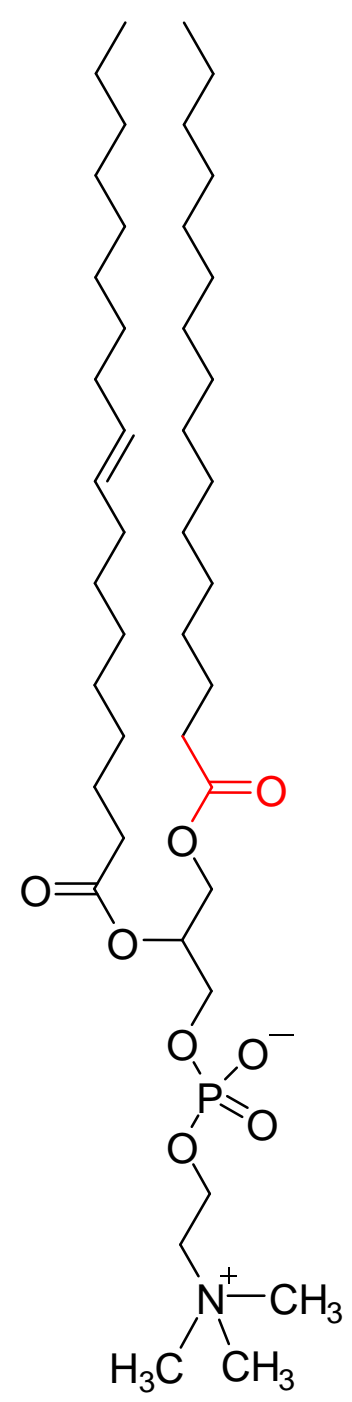

AOPC

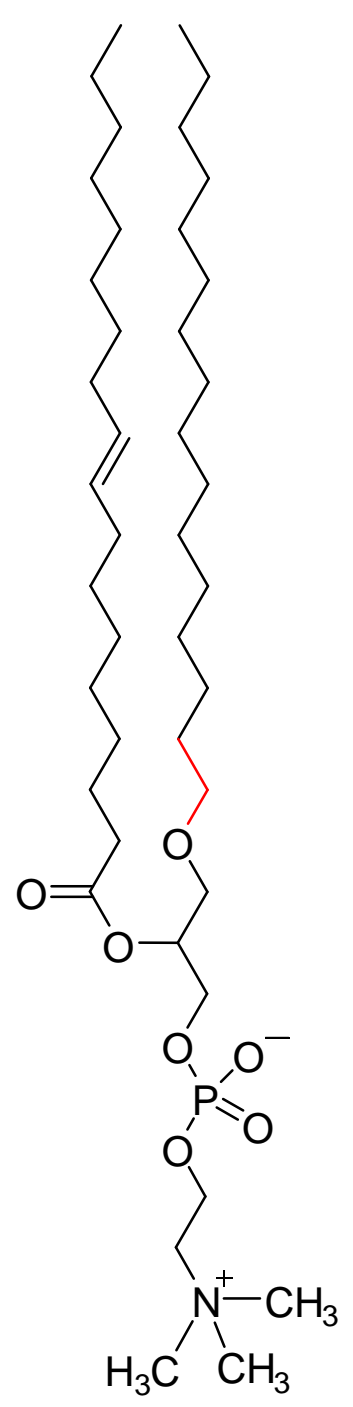

Scheme S1. Schematic representation of the chemical structure transformations from 'standard' CHARMM-GUI lipids (left) to their experimentally registered analogues present in the model membrane (right); the modified residues are colored in red 

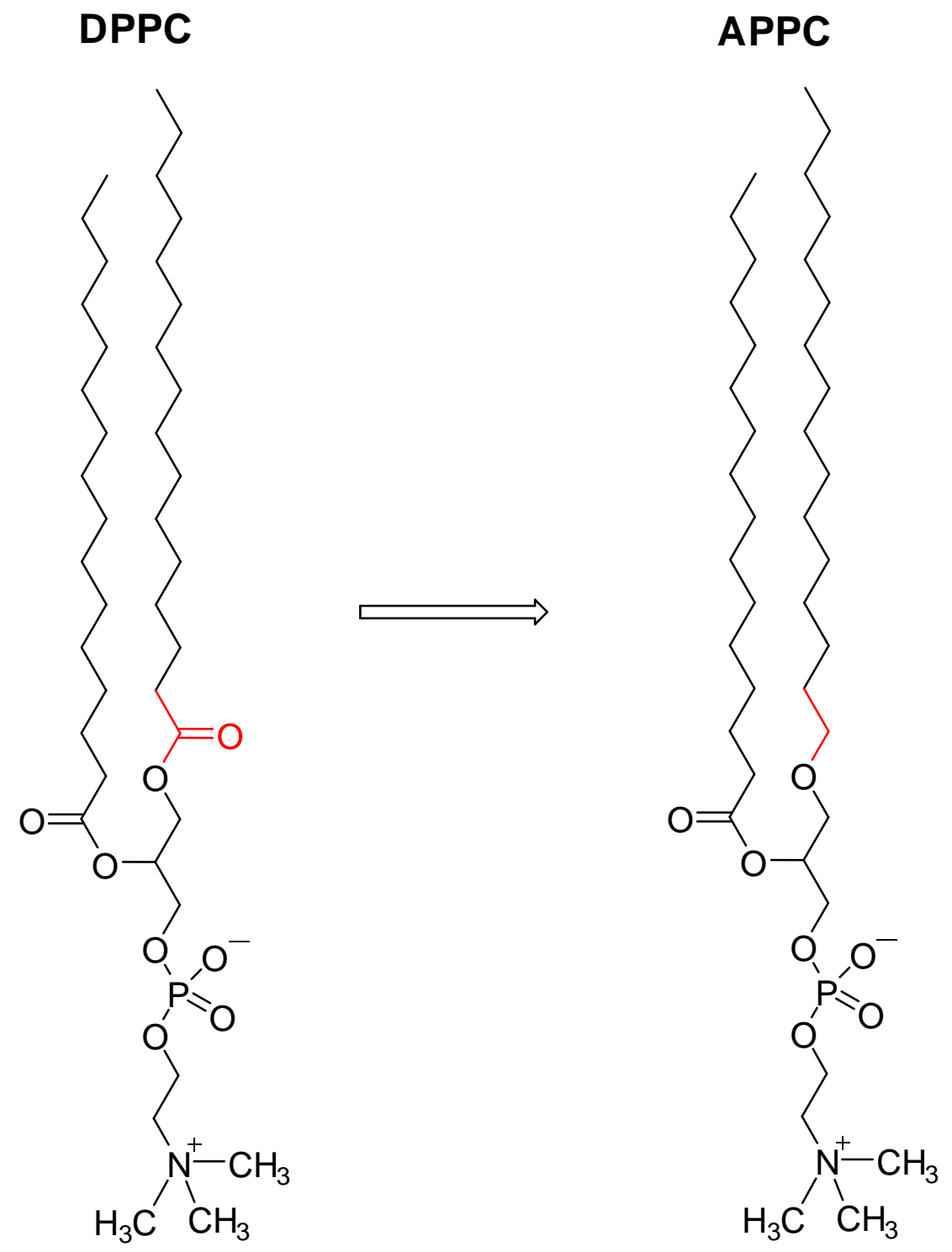

Scheme S1. (Continued) 

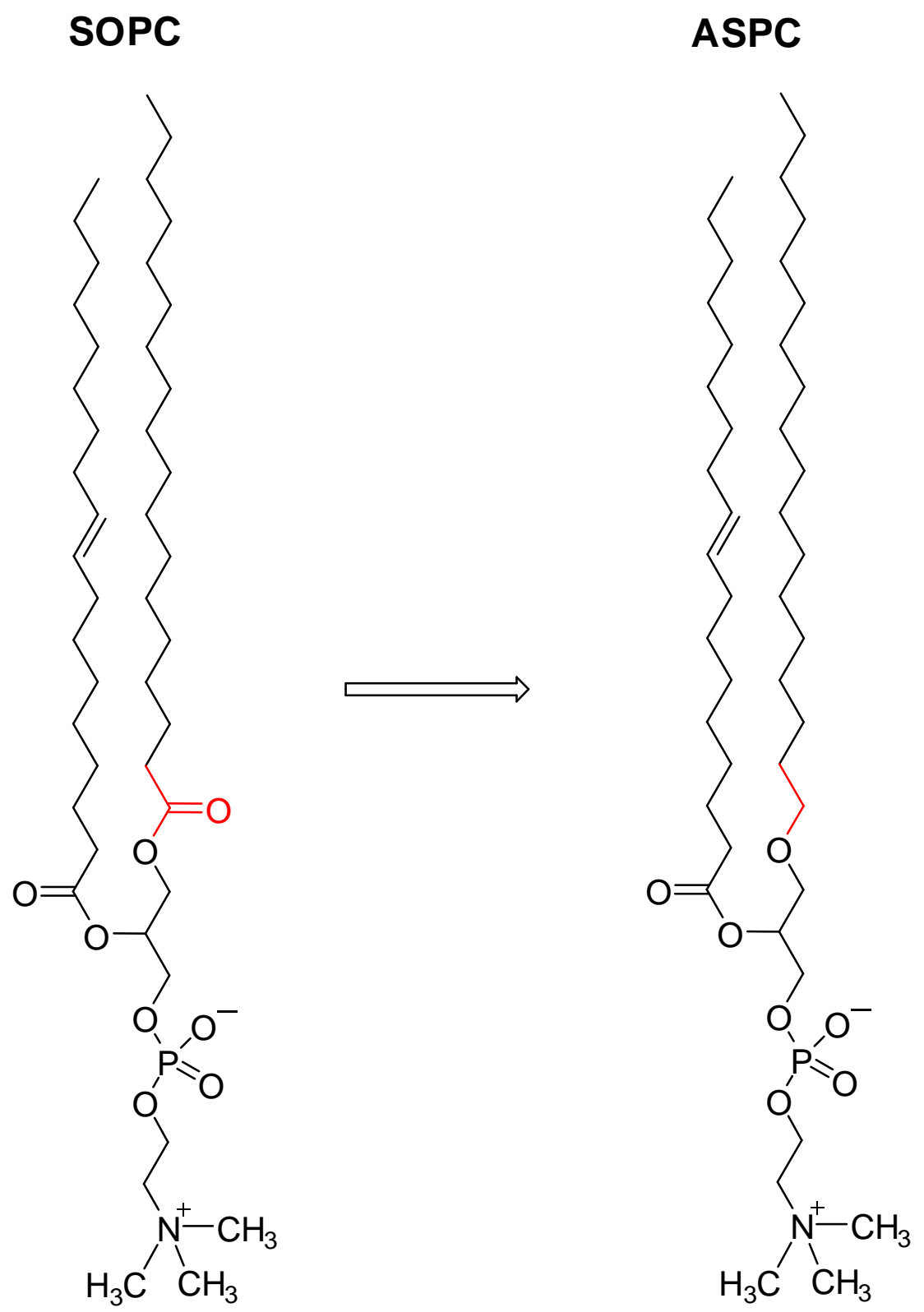

Scheme S1. (Continued) 

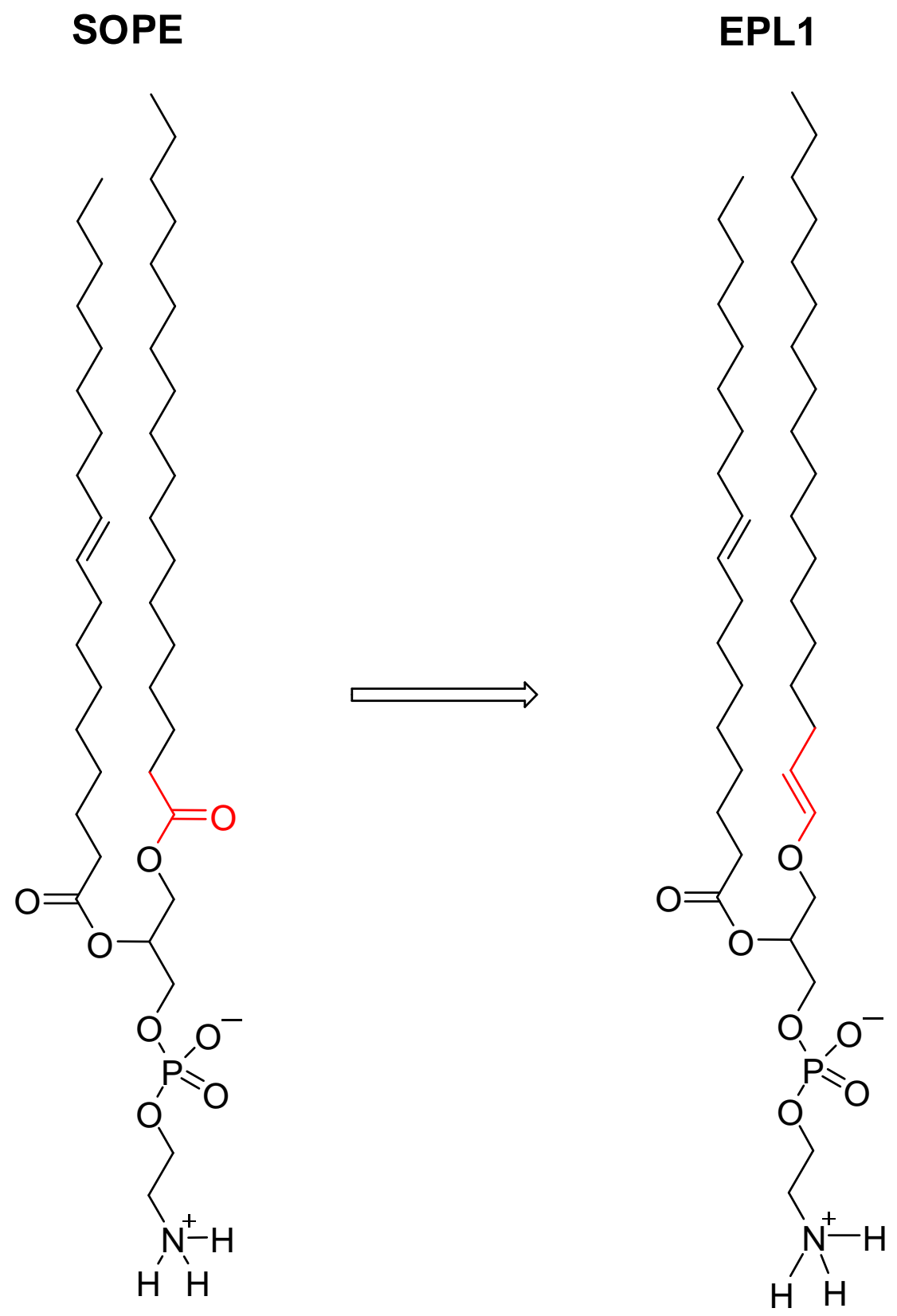

Scheme S1. (Continued) 


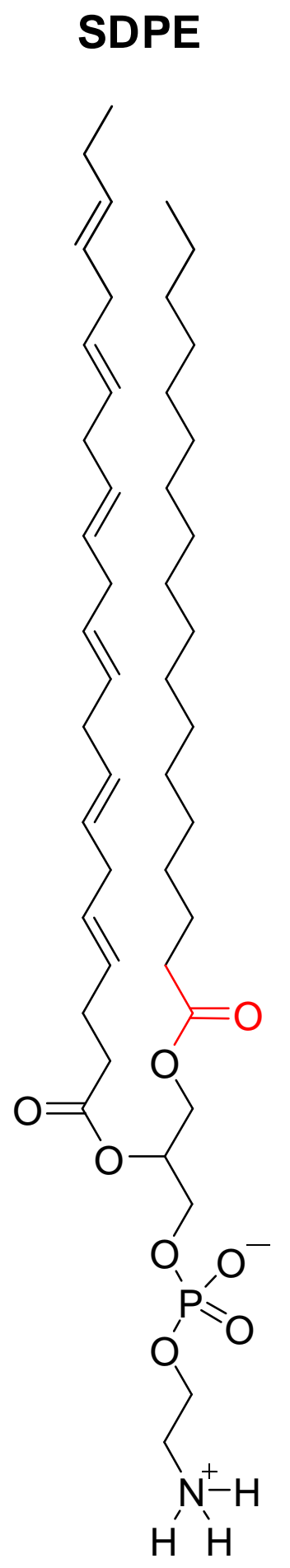

EPL2

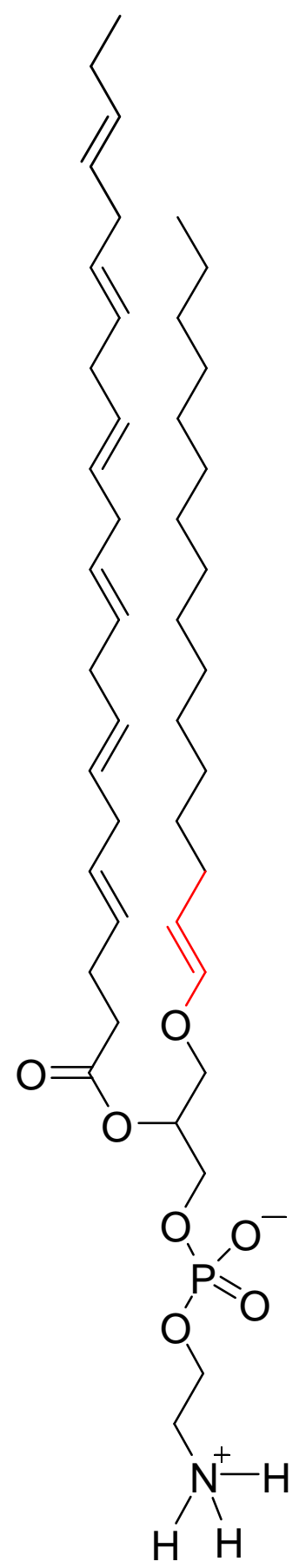

Scheme S1. (Continued) 

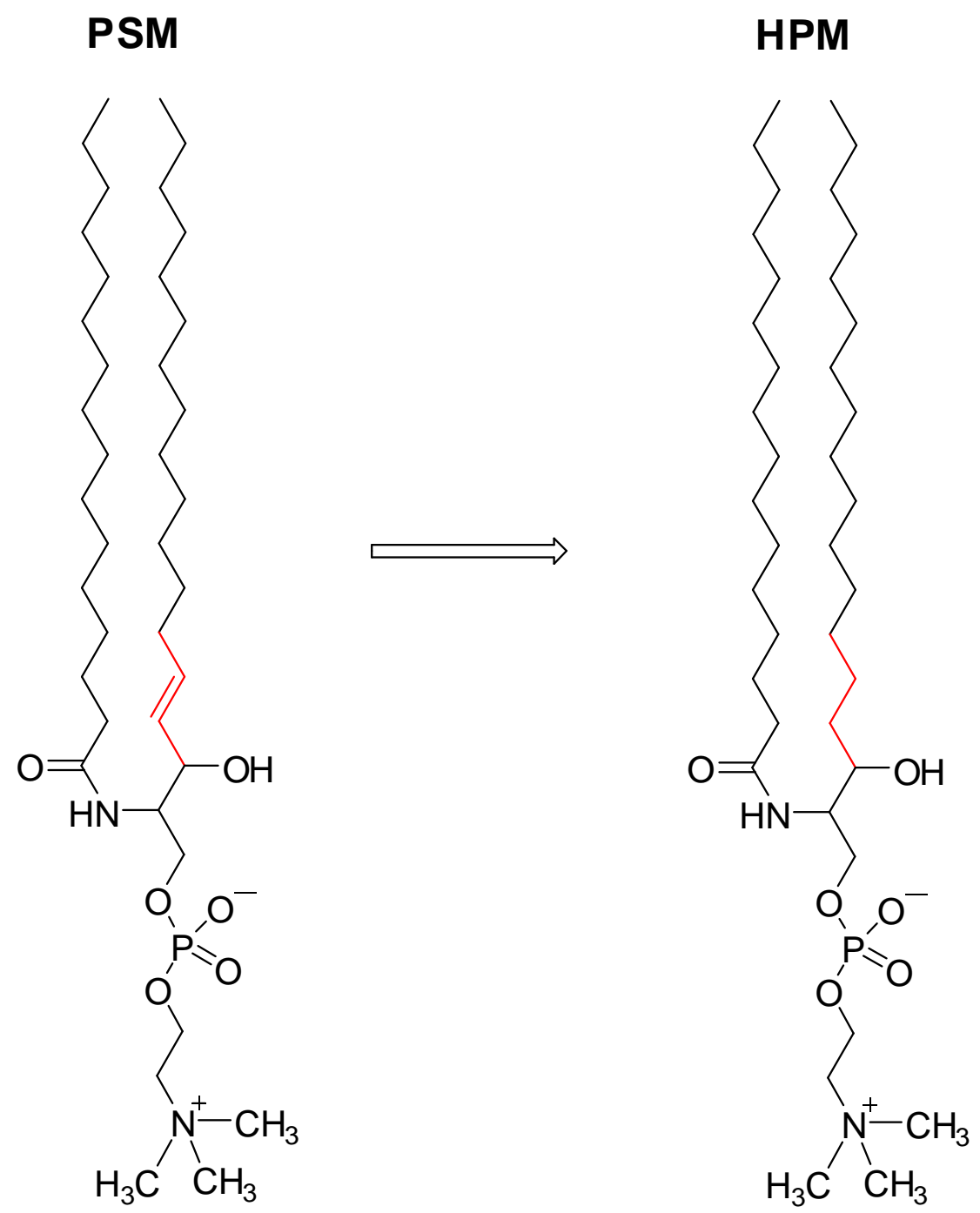

Scheme S1. (Continued) 

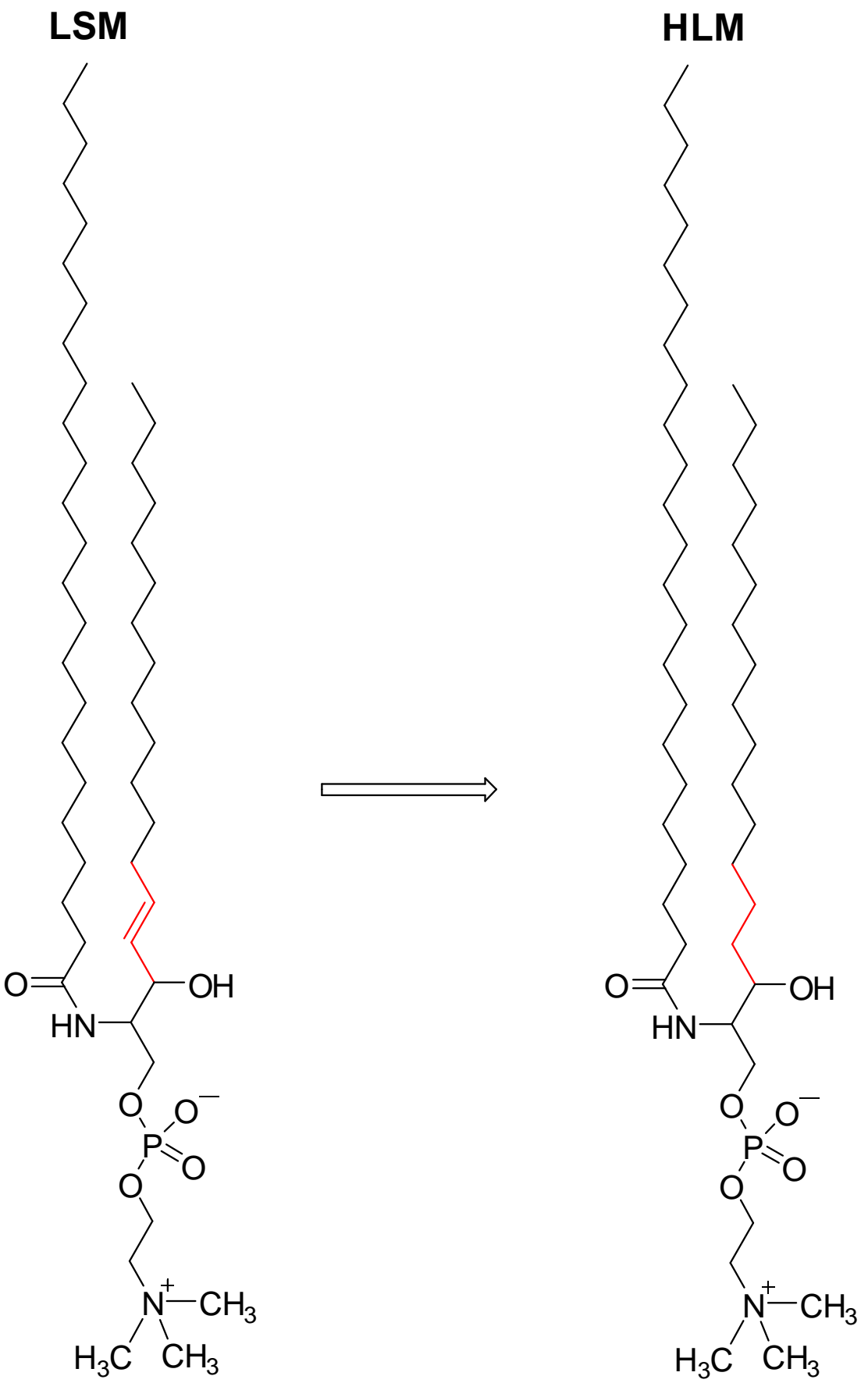

Scheme S1. (Continued) 
FOS10

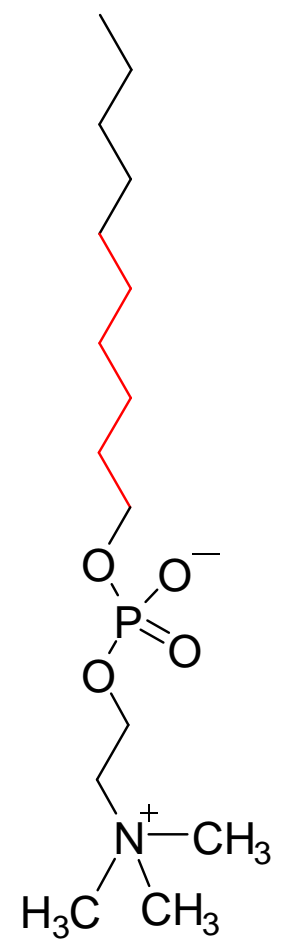

Scheme S1. (Continued)
SPC

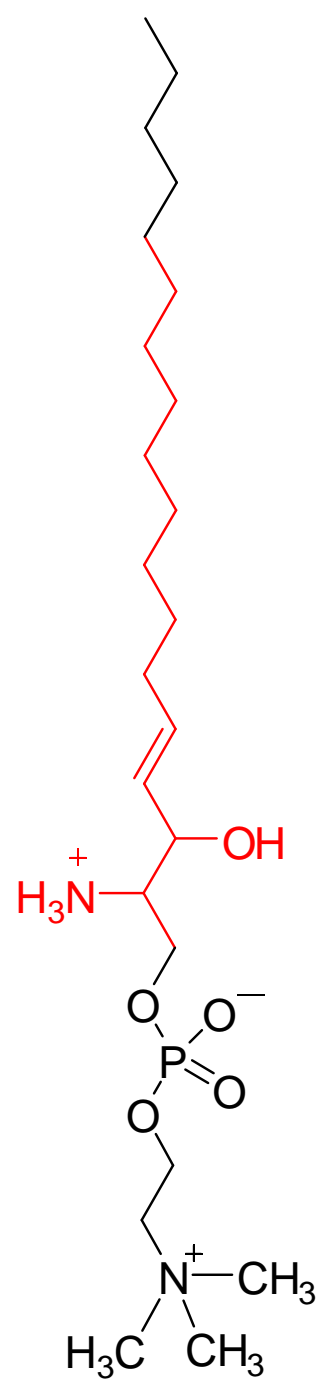


FOS10

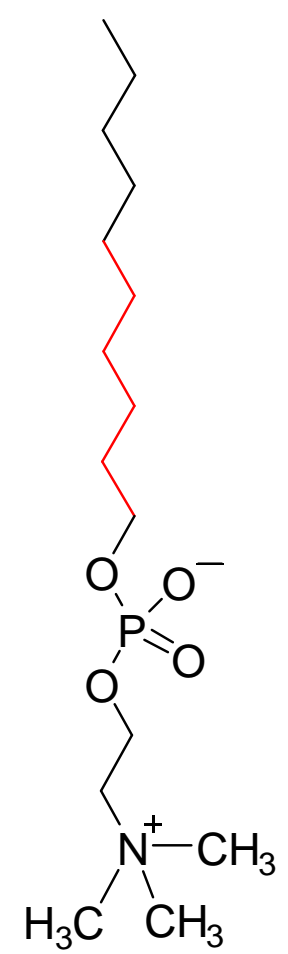

Scheme S1. (Continued)

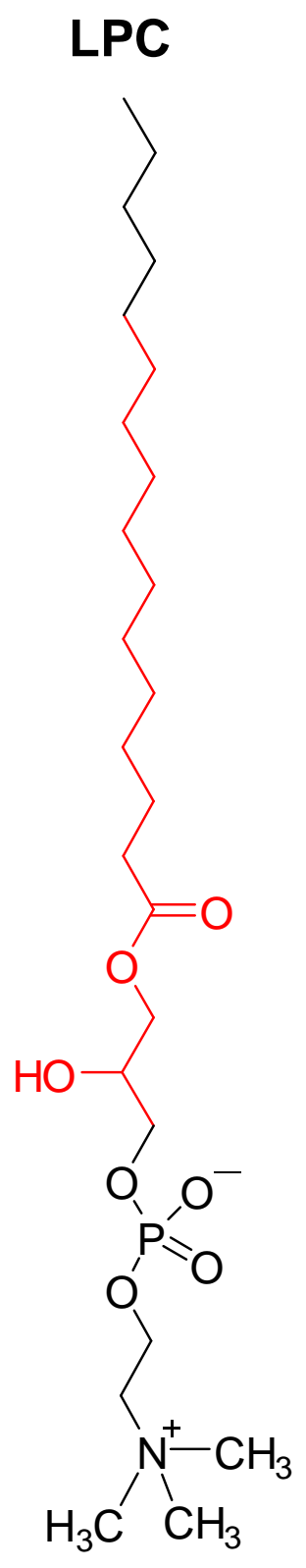


FOS10

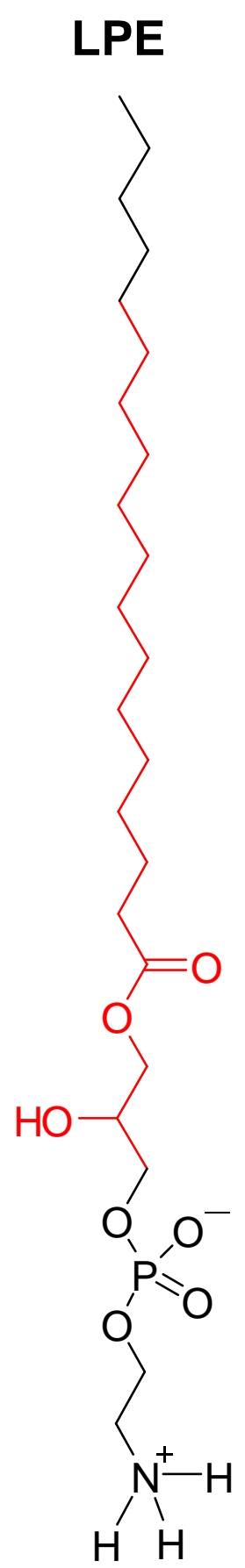

Scheme S1. (Continued) 


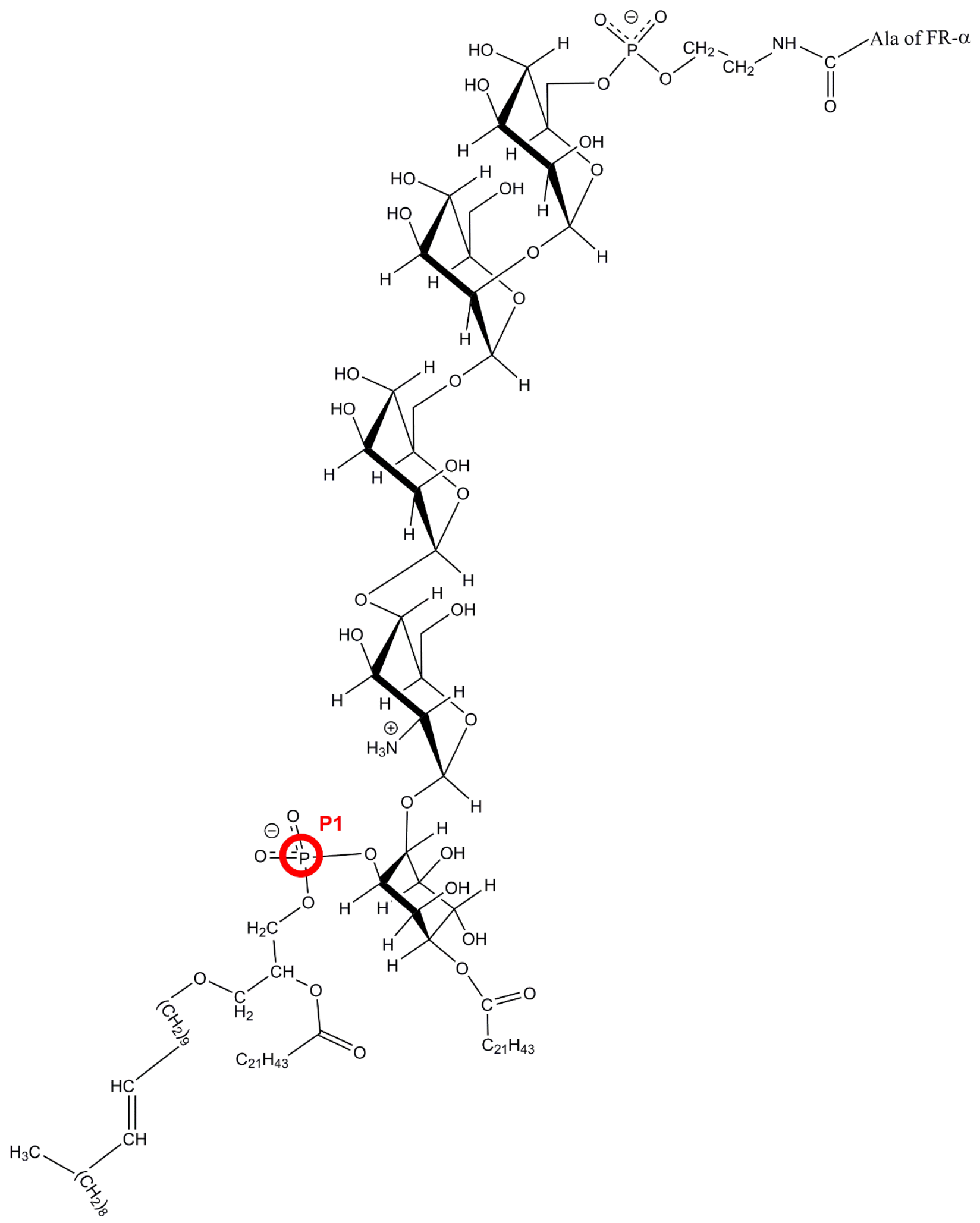

Figure S1. Chemical formula of the glycosyl-phosphatidylinositol anchor used to insert the receptor into the outer leaflet of the lipid bilayer 

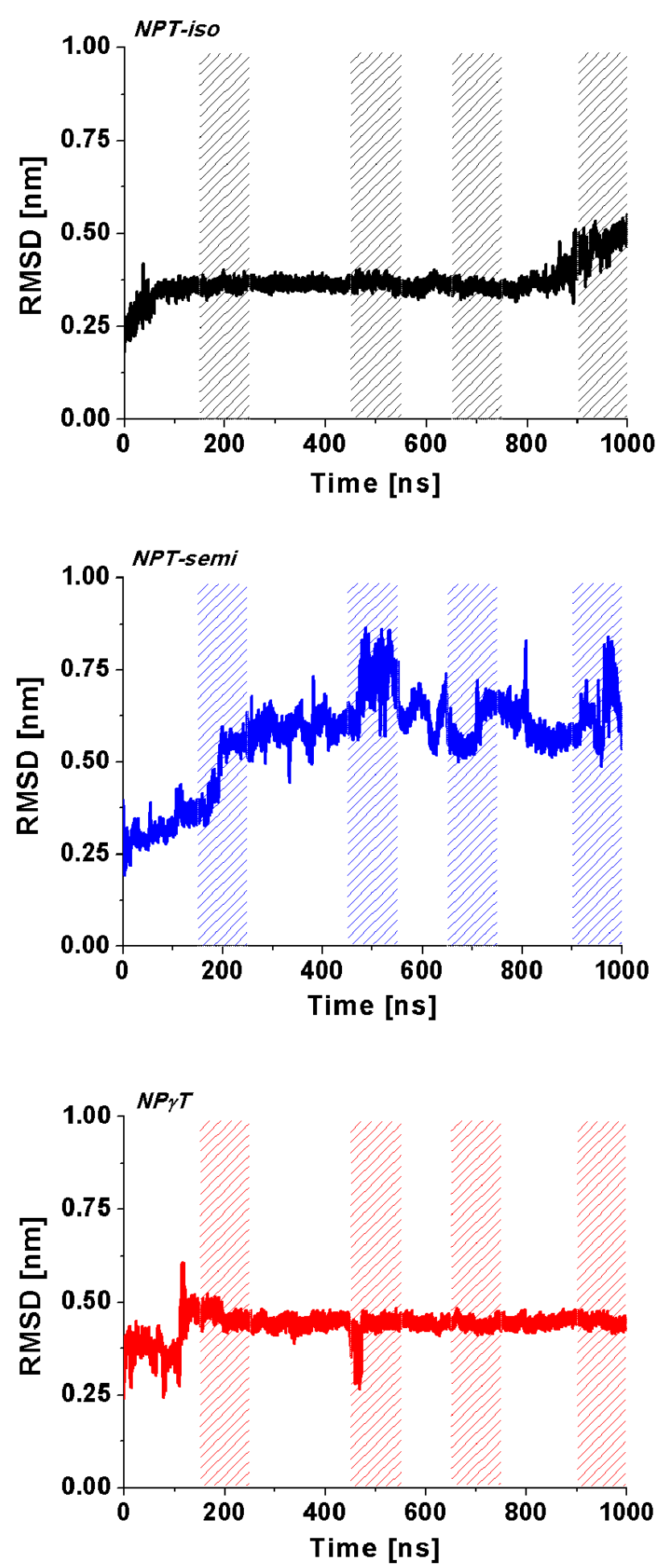

Figure S2. Evolution of the root-mean-square deviation of the atomic coordinates of the folate receptor- $\alpha$ with respect to the initial energy-minimized structure in the NPT-iso (top), NPT-semi (middle), and NP $\gamma \mathrm{T}$ (bottom) simulations; the four analyzed trajectory segments are shaded 
Mass and charge density profiles
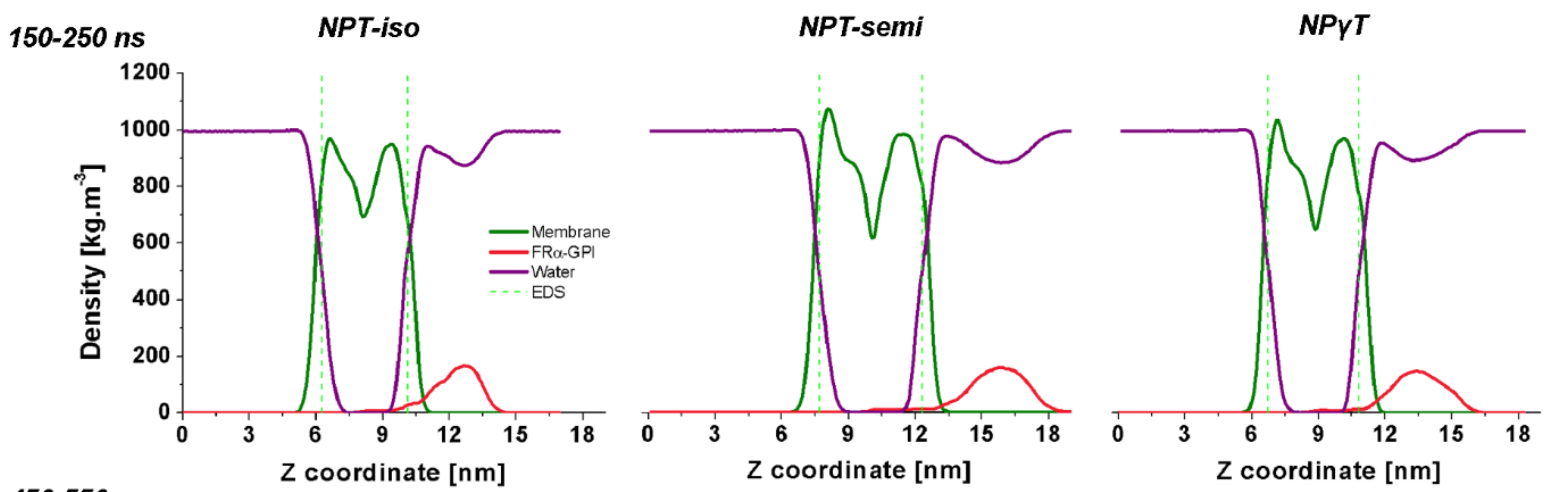

$450-550$ ns
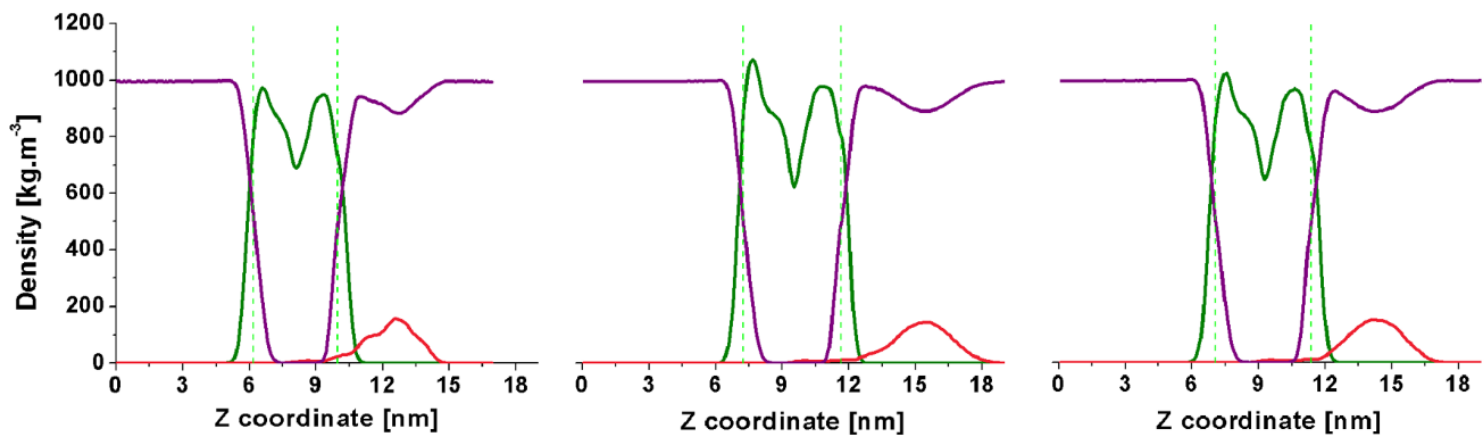

$650-750 \mathrm{~ns}$
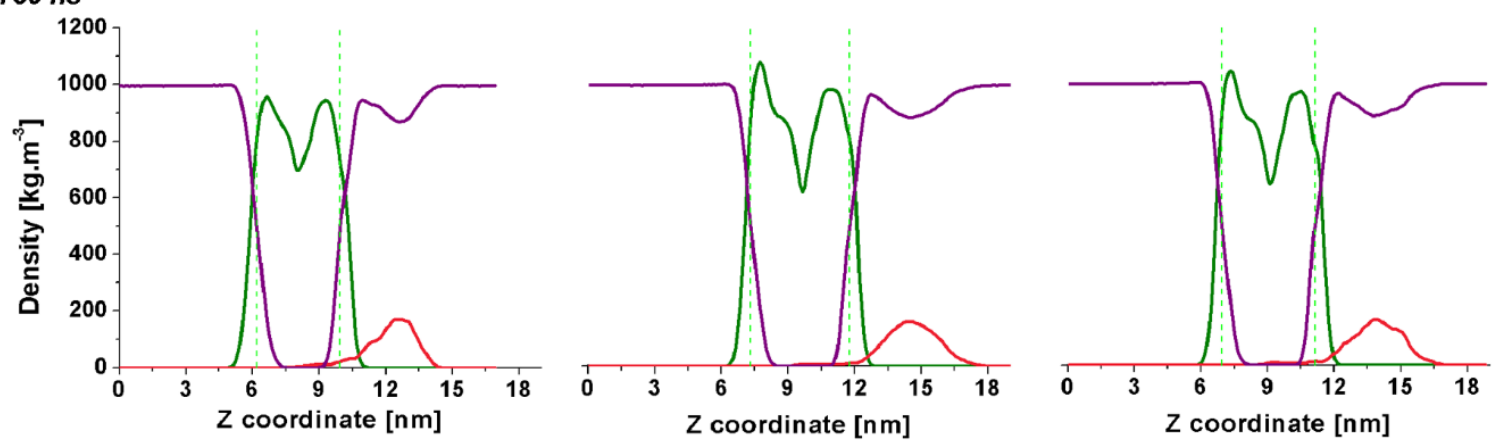

$900-1000 \mathrm{~ns}$
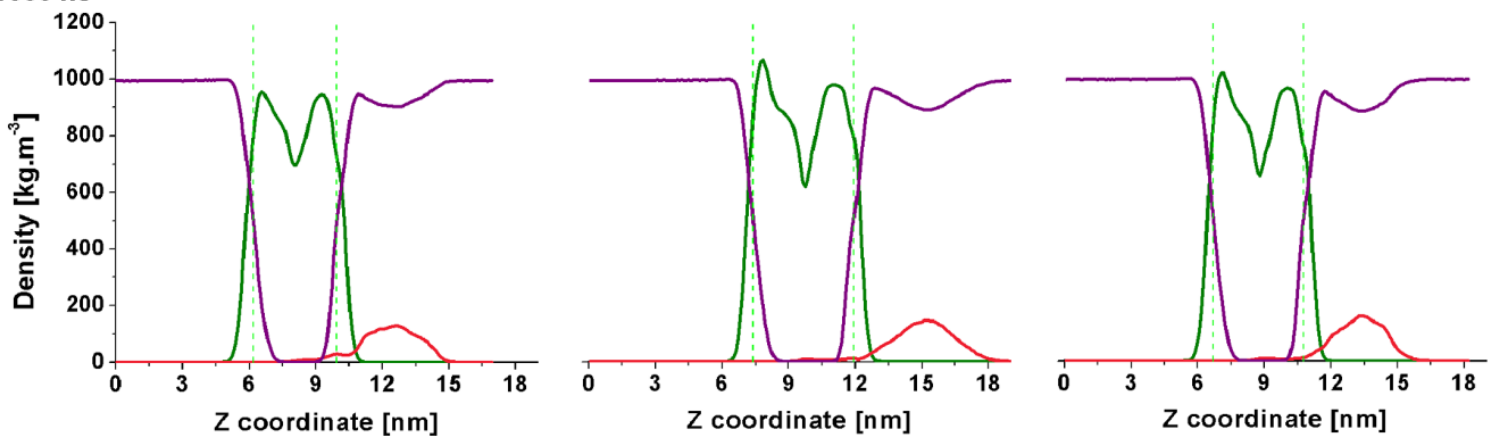

Figure S3. Mass density profiles in direction normal to the membrane in the four trajectory segments (from top to bottom) of the NPT-iso (left), NPT-semi (middle), and NP $\gamma$ T (right) simulations; the dashed green lines mark the equimolecular dividing surfaces 
The density of the lipid bilayer in NPT-iso is slightly lower than the one of the water molecules. The highest peak is for the inner leaflet in the 450-550 ns trajectory fragment -973 $\mathrm{kg} \cdot \mathrm{m}^{-3}$, and the average density of water is ca. $995 \mathrm{~kg} \cdot \mathrm{m}^{-3}$. Furthermore, the density of the two monolayers is similar. The difference is about $20 \mathrm{~kg} \cdot \mathrm{m}^{-3}$ in the first trajectory part (TP) and drops down to less than $10 \mathrm{~kg} \cdot \mathrm{m}^{-3}$ in the final one, mainly due to decrease of the value for the inner leaflet. Overall, the mass density profile of the lipids in NPT-iso signifies substantial disorder and practically melted state of the entire bilayer. The curve for FR $\alpha$-GPI looks similar for the first three TPs, with two maxima ca. $1 \mathrm{~nm}$ apart, one of which becomes more noticeable with time. In the 900-1000 ns trajectory segment the two peaks merge and form a broader profile, located closer to the membrane surface. This suggests movement of the receptor towards the lipid bilayer over time.

In the NPT-semi simulation, the two leaflets are clearly distinct in all TPs. The difference in the maxima of the two lipid peaks varies between 88 and $96 \mathrm{~kg} \cdot \mathrm{m}^{-3}$ in favor of the inner monolayer. The probable cause is better structuring of the inner leaflet (between 71 and $81 \mathrm{~kg} \cdot \mathrm{m}^{-3}$ denser than bulk water) due to the presence of charged lipid heads. This is qualitatively different from the behavior of the lipids in NPT-iso. The tighter packing is accompanied by specific ordering of the longer tails, evident from the shoulder in the lipid density curve of this leaflet (left green peak in Figure S3). The density of the outer monolayer is still lower than the one of the water environment. Overall, the semi-isotropic pressure scaling induces denser lipid packing in the membrane (the peaks of the lipid density exceed the corresponding ones in NPT-iso by more than $100 \mathrm{~kg} \cdot \mathrm{m}^{-3}$ for the inner and by over $30 \mathrm{~kg} \cdot \mathrm{m}^{-3}$ for the outer leaflet). This more expressed compression of the lipids in NPT-semi coincides with our previous observations on the effect of pressure scaling on DPPC or mixed bilayers [Ref. 25 of the main manuscript]. The peak for the anchored receptor is more symmetric and broad, which implies homogeneous fluctuations relative to the bilayer normal and elevated flexibility of the anchor. The part of the curve that overlaps with the equimolecular dividing surface is much smaller than in NPT-iso, indicating that the FR $\alpha$-GPI is positioned above the interface.

The behavior of the system in NP $\gamma \mathrm{T}$ is somewhat intermediate to the other two production runs. The shape of the curves is identical to that in NPT-semi - the characteristic structuring of the inner leaflet is still observed but is not so pronounced. The latter is verified by the slightly lower maximum lipid density - between 1016 and $1037 \mathrm{~kg} \cdot \mathrm{m}^{-3}$ for the inner and between 963 and 967 $\mathrm{kg} . \mathrm{m}^{-3}$ for the outer leaflet over the simulation. Similar to the other trajectories, the smallest values are realized in the final TP. The density profiles in this one imply liquid state of the membrane with some degree of ordering. The profile for FR $\alpha$-GPI in the first half of the simulation is broad and smooth. After $650 \mathrm{~ns}$ changes in the receptor occur that reshape the curve - it becomes slightly more structured. The protein is positioned near the lipids, but to a lesser degree than in NPT-iso and more than in NPT-semi. 


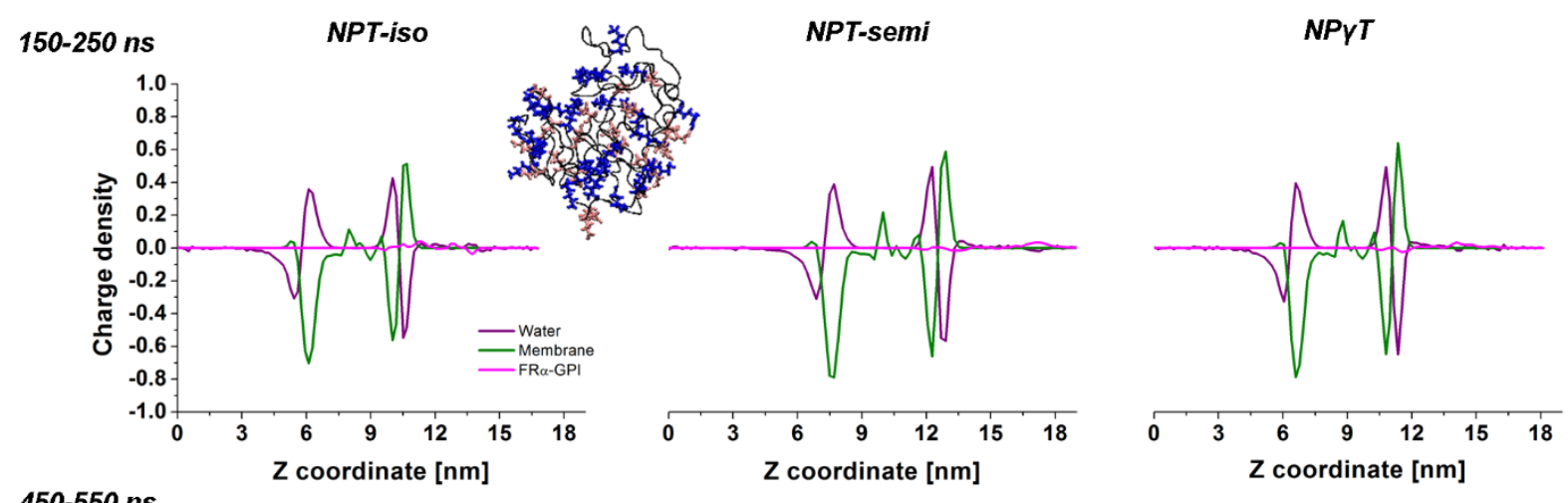

450-550 ns

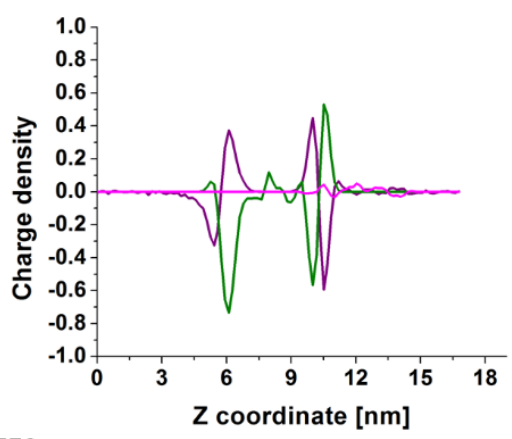

$650-750$ ns
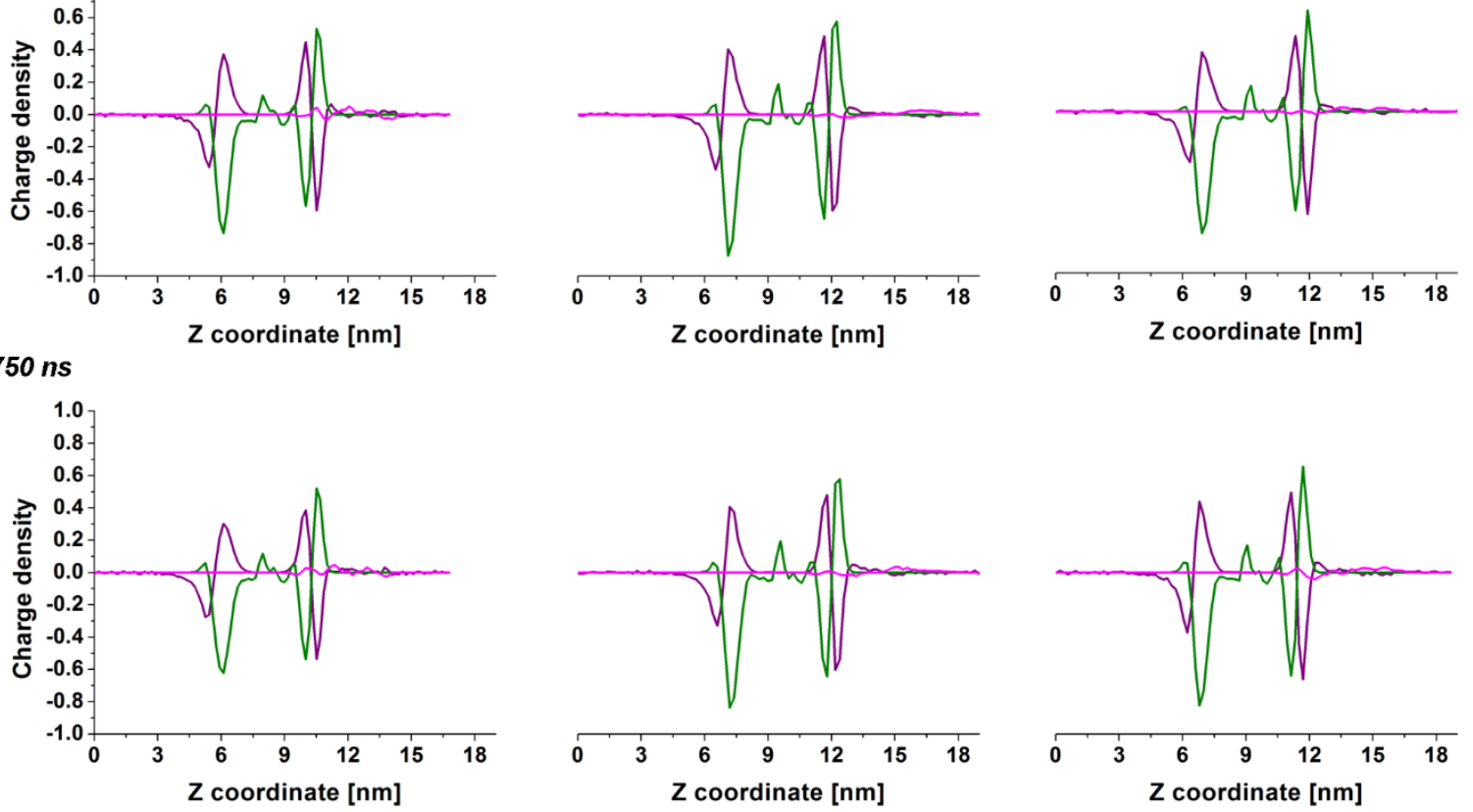

$900-1000 \mathrm{~ns}$
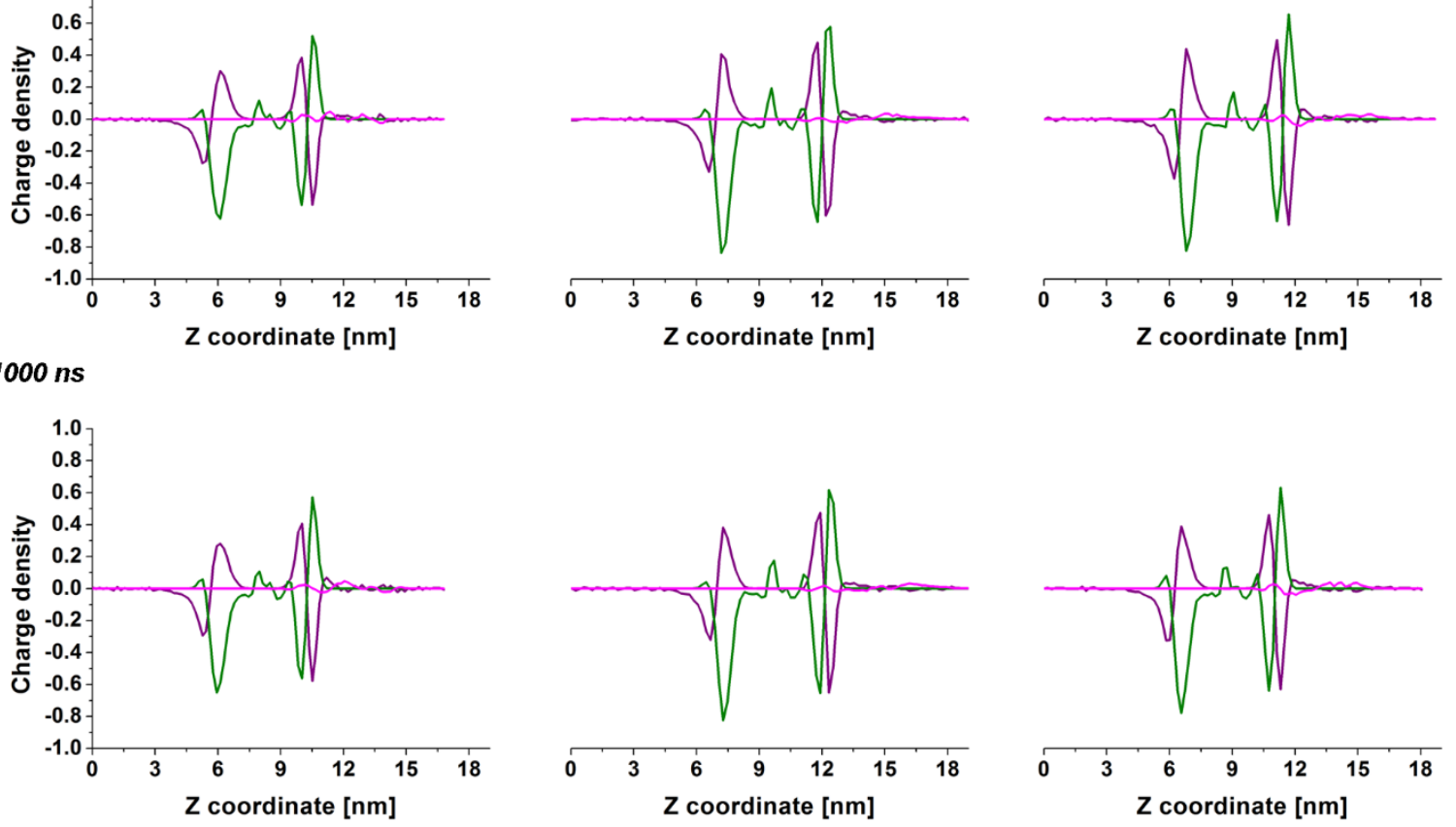

Figure S4. Charge density profiles in direction normal to the membrane interface in the four trajectory segments (from top to bottom) of the NPT-iso (left), NPT-semi (middle), and NP $\gamma \mathrm{T}$ (right) simulations; the inset at the top shows the location of the positively (pink) and negatively (blue) charged residues along the protein structure 

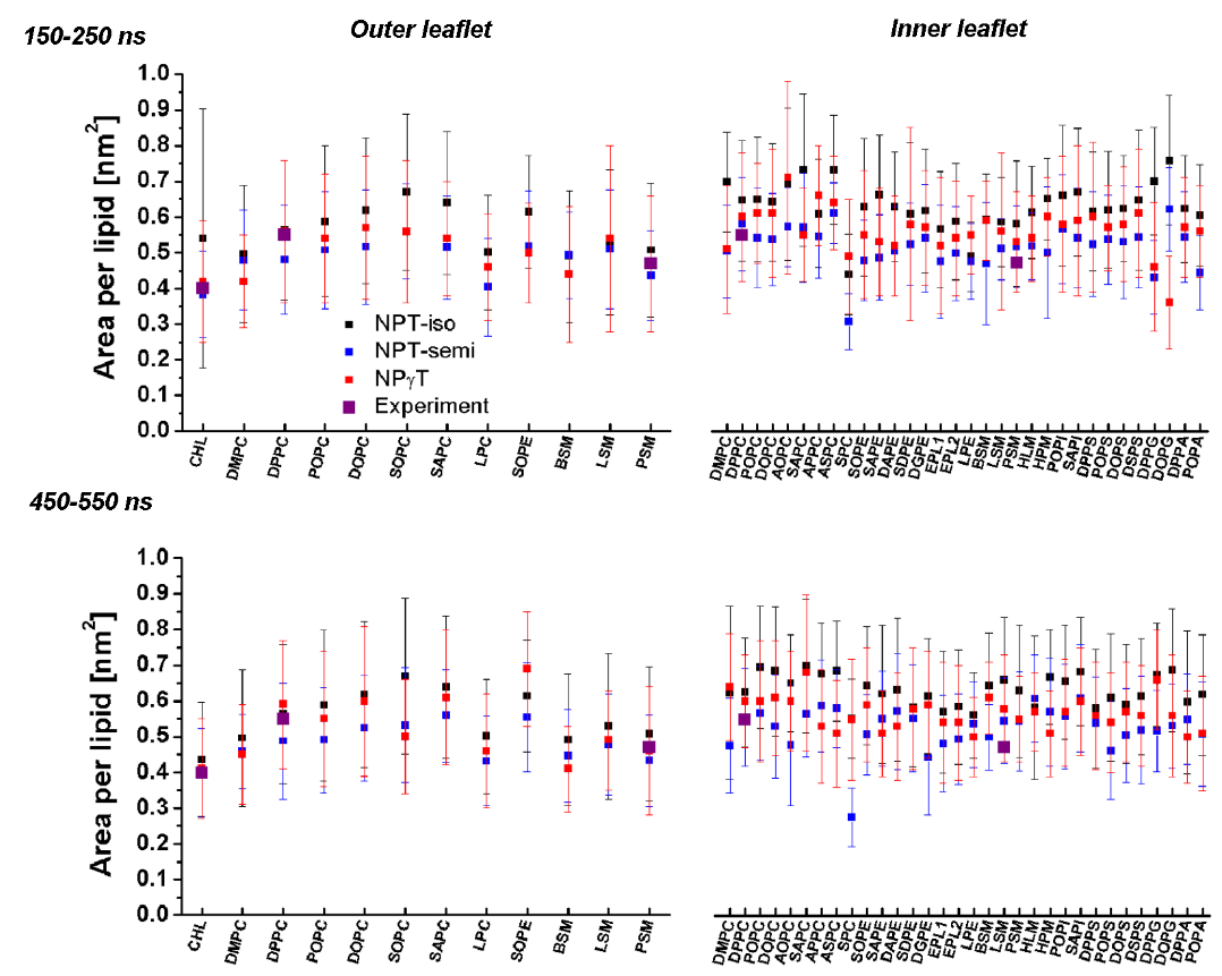

$650-750 \mathrm{~ns}$
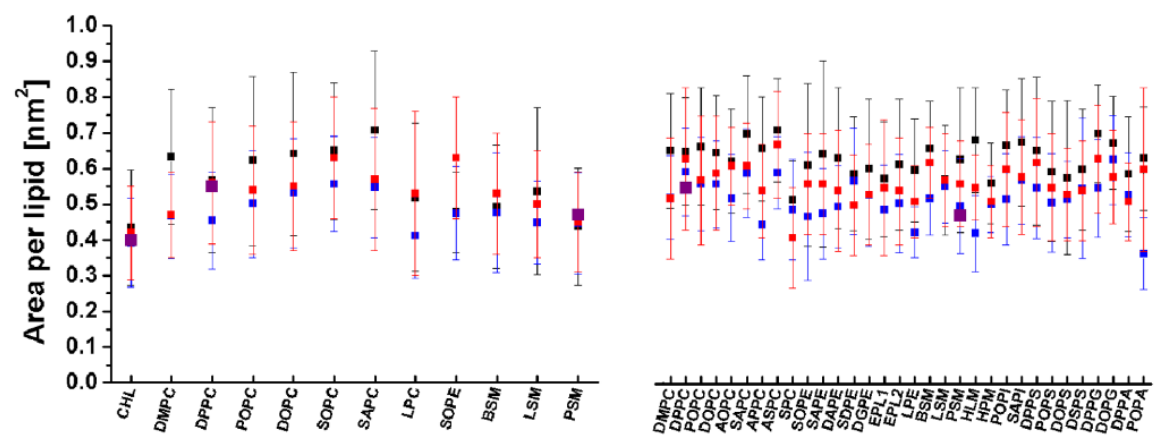

$900-1000 \mathrm{~ns}$
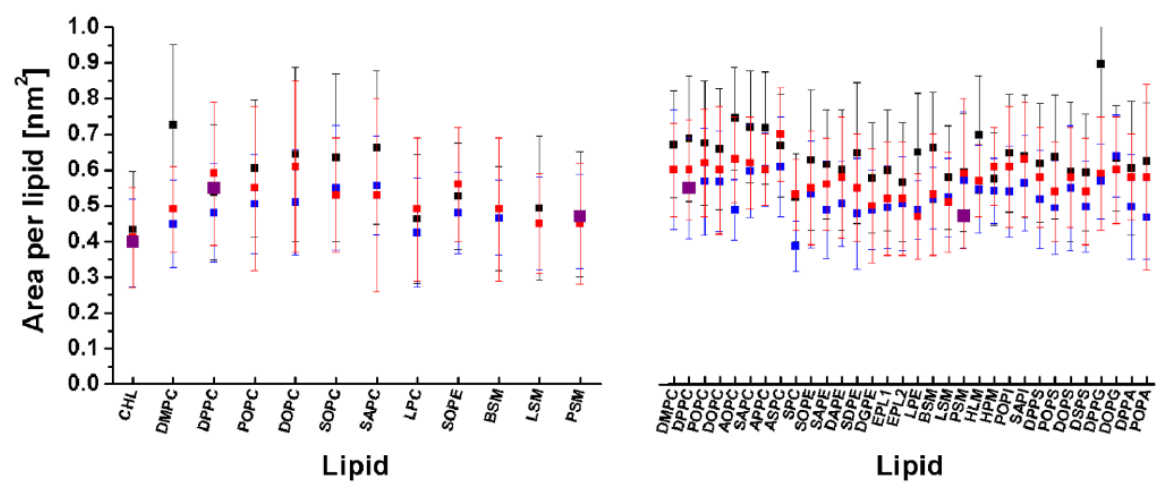

Figure S5. Average values (with standard deviations) of the area per lipid in the outer (left) and inner (right) leaflet of the membrane in the four trajectory segments (from top to bottom) of the NPT-iso, NPT-semi, and NP $\gamma$ T simulations; the experimental areas of CHL, DPPC, and PSM are denoted as purple squares 
Table S1. Average values (with standard deviations) of the area per lipid in the outer and in the inner monolayer of the membrane in the NPT-iso, NPT-semi, and NP $\gamma$ T simulations in the 150250 ns trajectory segment

\begin{tabular}{|c|c|c|c|c|c|c|c|}
\hline \multicolumn{4}{|c|}{ Outer monolayer } & \multicolumn{4}{|c|}{ Inner monolayer } \\
\hline & NPT-iso & NPT-semi & $\mathbf{N P} \gamma \mathbf{T}$ & & NPT-iso & NPT-semi & $\mathbf{N P} \gamma \mathbf{T}$ \\
\hline Lipid & Area $\left[\mathrm{nm}^{2}\right]$ & Area $\left[\mathrm{nm}^{2}\right]$ & Area $\left[\mathrm{nm}^{2}\right]$ & Lipid & Area $\left[\mathrm{nm}^{2}\right]$ & Area $\left[\mathrm{nm}^{2}\right]$ & Area $\left[\mathrm{nm}^{2}\right]$ \\
\hline CHL & $0.54 \pm 0.36$ & $0.38 \pm 0.12$ & $0.42 \pm 0.17$ & CHL & ----- & ----- & ----- \\
\hline DMPC & $0.50 \pm 0.19$ & $0.48 \pm 0.14$ & $0.42 \pm 0.13$ & DMPC & $0.70 \pm 0.14$ & $0.50 \pm 0.13$ & $0.51 \pm 0.18$ \\
\hline DPPC & $0.56 \pm 0.19$ & $0.48 \pm 0.15$ & $0.56 \pm 0.20$ & DPPC & $0.65 \pm 0.17$ & $0.58 \pm 0.13$ & $0.60 \pm 0.18$ \\
\hline POPC & $0.59 \pm 0.21$ & $0.51 \pm 0.17$ & $0.54 \pm 0.18$ & POPC & $0.65 \pm 0.17$ & $0.54 \pm 0.14$ & $0.61 \pm 0.14$ \\
\hline DOPC & $0.62 \pm 0.20$ & $0.52 \pm 0.16$ & $0.57 \pm 0.20$ & DOPC & $0.64 \pm 0.16$ & $0.54 \pm 0.13$ & $0.61 \pm 0.18$ \\
\hline SOPC & $0.67 \pm 0.21$ & $0.56 \pm 0.13$ & $0.56 \pm 0.20$ & SOPC & ----- & ----- & ----- \\
\hline AOPC & ----- & ----- & ----- & AOPC & $0.69 \pm 0.21$ & $0.57 \pm 0.11$ & $0.71 \pm 0.27$ \\
\hline SAPC & $0.64 \pm 0.20$ & $0.51 \pm 0.15$ & $0.54 \pm 0.16$ & SAPC & $0.73 \pm 0.21$ & $0.57 \pm 0.15$ & $0.55 \pm 0.13$ \\
\hline APPC & ----- & ----- & ----- & APPC & $0.61 \pm 0.15$ & $0.54 \pm 0.12$ & $0.66 \pm 0.14$ \\
\hline ASPC & $\begin{array}{ll}---- \\
\end{array}$ & ----- & ----- & ASPC & $0.73 \pm 0.15$ & $0.61 \pm 0.08$ & $0.64 \pm 0.13$ \\
\hline LPC & $0.50 \pm 0.16$ & $0.40 \pm 0.14$ & $0.46 \pm 0.15$ & LPC & ----- & ----- & $\begin{array}{l}---- \\
\end{array}$ \\
\hline SPC & $\begin{array}{l}---- \\
\end{array}$ & $\begin{array}{l}---- \\
\end{array}$ & $\begin{array}{l}---- \\
\end{array}$ & SPC & $0.44 \pm 0.11$ & $0.31 \pm 0.08$ & $0.49 \pm 0.16$ \\
\hline SOPE & $0.61 \pm 0.15$ & $0.52 \pm 0.16$ & $0.50 \pm 0.14$ & SOPE & $0.63 \pm 0.19$ & $0.48 \pm 0.11$ & $0.55 \pm 0.18$ \\
\hline SAPE & ----- & ----- & ----- & SAPE & $0.66 \pm 0.15$ & $0.49 \pm 0.12$ & $0.53 \pm 0.15$ \\
\hline DAPE & ----- & ----- & ----- & DAPE & $0.63 \pm 0.19$ & $0.50 \pm 0.13$ & $0.52 \pm 0.14$ \\
\hline SDPE & ----- & ----- & ----- & SDPE & $0.61 \pm 0.17$ & $0.52 \pm 0.11$ & $0.58 \pm 0.27$ \\
\hline DGPE & ----- & ----- & ----- & DGPE & $0.62 \pm 0.16$ & $0.54 \pm 0.15$ & $0.57 \pm 0.16$ \\
\hline EPL1 & ----- & ----- & ----- & EPL1 & $0.56 \pm 0.16$ & $0.48 \pm 0.16$ & $0.52 \pm 0.19$ \\
\hline EPL2 & ----- & ----- & ----- & EPL2 & $0.59 \pm 0.10$ & $0.50 \pm 0.13$ & $0.54 \pm 0.16$ \\
\hline LPE & ----- & ----- & ----- & LPE & $0.49 \pm 0.12$ & $0.47 \pm 0.11$ & $0.55 \pm 0.11$ \\
\hline BSM & $0.49 \pm 0.18$ & $0.49 \pm 0.12$ & $0.44 \pm 0.19$ & BSM & $0.59 \pm 0.12$ & $0.47 \pm 0.17$ & $0.59 \pm 0.11$ \\
\hline LSM & $0.53 \pm 0.20$ & $0.51 \pm 0.17$ & $0.54 \pm 0.26$ & LSM & $0.58 \pm 0.17$ & $0.51 \pm 0.09$ & $0.56 \pm 0.22$ \\
\hline PSM & $0.51 \pm 0.19$ & $0.44 \pm 0.12$ & $0.47 \pm 0.19$ & PSM & $0.58 \pm 0.12$ & $0.52 \pm 0.11$ & $0.53 \pm 0.14$ \\
\hline HLM & ----- & ----- & ----- & HLM & $0.61 \pm 0.11$ & $0.52 \pm 0.09$ & $0.54 \pm 0.12$ \\
\hline HPM & $\begin{array}{l}---- \\
\end{array}$ & $\begin{array}{l}---- \\
\end{array}$ & $\begin{array}{l}---- \\
\end{array}$ & HPM & $0.65 \pm 0.19$ & $0.50 \pm 0.19$ & $0.60 \pm 0.11$ \\
\hline POPI & ----- & ----- & ----- & POPI & $0.66 \pm 0.17$ & $0.57 \pm 0.15$ & $0.58 \pm 0.19$ \\
\hline SAPI & ----- & ----- & ----- & SAPI & $0.67 \pm 0.17$ & $0.54 \pm 0.14$ & $0.59 \pm 0.21$ \\
\hline DPPS & ----- & ----- & ----- & DPPS & $0.62 \pm 0.16$ & $0.52 \pm 0.15$ & $0.60 \pm 0.21$ \\
\hline POPS & ----- & $\begin{array}{l}---- \\
\end{array}$ & $\begin{array}{l}---- \\
\end{array}$ & POPS & $0.62 \pm 0.14$ & $0.54 \pm 0.13$ & $0.57 \pm 0.12$ \\
\hline DOPS & ----- & ----- & ----- & DOPS & $0.62 \pm 0.20$ & $0.53 \pm 0.16$ & $0.58 \pm 0.16$ \\
\hline DSPS & ----- & ----- & ----- & DSPS & $0.65 \pm 0.15$ & $0.54 \pm 0.14$ & $0.61 \pm 0.18$ \\
\hline DPPG & ----- & ----- & ----- & DPPG & $0.70 \pm 0.18$ & $0.43 \pm 0.10$ & $0.46 \pm 0.18$ \\
\hline DOPG & $\begin{array}{l}---- \\
\end{array}$ & $\begin{array}{l}---- \\
\end{array}$ & $\begin{array}{l}---- \\
\end{array}$ & DOPG & $0.76 \pm 0.15$ & $0.62 \pm 0.12$ & $0.36 \pm 0.13$ \\
\hline DPPA & ----- & ----- & ----- & DPPA & $0.62 \pm 0.15$ & $0.54 \pm 0.13$ & $0.57 \pm 0.14$ \\
\hline POPA & ----- & ----- & ----- & POPA & $0.60 \pm 0.15$ & $0.44 \pm 0.10$ & $0.56 \pm 0.13$ \\
\hline
\end{tabular}


Table S2. Average values (with standard deviations) of the area per lipid in the outer and in the inner monolayer of the membrane in the NPT-iso, NPT-semi, and NP $\gamma \mathrm{T}$ simulations in the 450$550 \mathrm{~ns}$ trajectory segment

\begin{tabular}{|c|c|c|c|c|c|c|c|}
\hline \multicolumn{4}{|c|}{ Outer monolayer } & \multicolumn{4}{|c|}{ Inner monolayer } \\
\hline & NPT-iso & NPT-semi & $\mathbf{N P} \gamma \mathbf{T}$ & & NPT-iso & NPT-semi & $\mathbf{N P} \gamma \mathbf{T}$ \\
\hline Lipid & Area $\left[\mathrm{nm}^{2}\right]$ & Area $\left[\mathrm{nm}^{2}\right]$ & Area $\left[\mathrm{nm}^{2}\right]$ & Lipid & Area $\left[\mathrm{nm}^{2}\right]$ & Area $\left[\mathrm{nm}^{2}\right]$ & Area $\left[\mathrm{nm}^{2}\right]$ \\
\hline CHL & $0.44 \pm 0.18$ & $0.40 \pm 0.12$ & $0.41 \pm 0.14$ & CHL & ----- & ----- & ----- \\
\hline DMPC & $0.50 \pm 0.20$ & $0.46 \pm 0.10$ & $0.45 \pm 0.14$ & DMPC & $0.62 \pm 0.24$ & $0.48 \pm 0.13$ & $0.64 \pm 0.15$ \\
\hline DPPC & $0.56 \pm 0.13$ & $0.49 \pm 0.16$ & $0.59 \pm 0.18$ & DPPC & $0.66 \pm 0.15$ & $0.56 \pm 0.14$ & $0.60 \pm 0.13$ \\
\hline POPC & $0.59 \pm 0.16$ & $0.49 \pm 0.15$ & $0.55 \pm 0.19$ & POPC & $0.70 \pm 0.17$ & $0.57 \pm 0.13$ & $0.60 \pm 0.17$ \\
\hline DOPC & $0.62 \pm 0.15$ & $0.52 \pm 0.15$ & $0.60 \pm 0.21$ & DOPC & $0.68 \pm 0.17$ & $0.53 \pm 0.14$ & $0.61 \pm 0.16$ \\
\hline SOPC & $0.67 \pm 0.16$ & $0.53 \pm 0.16$ & $0.50 \pm 0.16$ & SOPC & ----- & ----- & ----- \\
\hline AOPC & ----- & ----- & ----- & AOPC & $0.65 \pm 0.13$ & $0.48 \pm 0.17$ & $0.60 \pm 0.14$ \\
\hline SAPC & $0.64 \pm 0.17$ & $0.56 \pm 0.13$ & $0.61 \pm 0.19$ & SAPC & $0.70 \pm 0.18$ & $0.56 \pm 0.12$ & $0.68 \pm 0.22$ \\
\hline APPC & $\begin{array}{ll}---- \\
\end{array}$ & $\begin{array}{ll}---- \\
\end{array}$ & $\begin{array}{ll}---- \\
\end{array}$ & APPC & $0.67 \pm 0.14$ & $0.59 \pm 0.13$ & $0.53 \pm 0.16$ \\
\hline ASPC & $\begin{array}{ll}---- \\
\end{array}$ & $\begin{array}{ll}---- \\
\end{array}$ & $\begin{array}{ll}---- \\
--\end{array}$ & ASPC & $0.69 \pm 0.14$ & $0.58 \pm 0.11$ & $0.51 \pm 0.15$ \\
\hline LPC & $0.50 \pm 0.16$ & $0.43 \pm 0.13$ & $0.46 \pm 0.16$ & LPC & ----- & ----- & ----- \\
\hline SPC & ----- & $\begin{array}{ll}---- \\
\end{array}$ & $\begin{array}{ll}---- \\
\end{array}$ & SPC & $0.55 \pm 0.11$ & $0.28 \pm 0.08$ & $0.55 \pm 0.17$ \\
\hline SOPE & $0.61 \pm 0.14$ & $0.55 \pm 0.15$ & $0.69 \pm 0.16$ & SOPE & $0.65 \pm 0.16$ & $0.51 \pm 0.11$ & $0.59 \pm 0.16$ \\
\hline SAPE & $\begin{array}{l}---- \\
\end{array}$ & ----- & $\begin{array}{l}---- \\
\end{array}$ & SAPE & $0.62 \pm 0.19$ & $0.55 \pm 0.13$ & $0.51 \pm 0.12$ \\
\hline DAPE & $\begin{array}{l}---- \\
\end{array}$ & $\begin{array}{l}---- \\
\end{array}$ & $\begin{array}{ll}---- \\
\end{array}$ & DAPE & $0.63 \pm 0.20$ & $0.57 \pm 0.16$ & $0.53 \pm 0.15$ \\
\hline SDPE & $\begin{array}{ll}---- \\
\end{array}$ & $\begin{array}{ll}---- \\
\end{array}$ & $\begin{array}{ll}---- \\
\end{array}$ & SDPE & $0.58 \pm 0.16$ & $0.55 \pm 0.15$ & $0.58 \pm 0.17$ \\
\hline DGPE & $\begin{array}{l}---- \\
\end{array}$ & $\begin{array}{l}---- \\
\end{array}$ & $\begin{array}{l}---- \\
\end{array}$ & DGPE & $0.61 \pm 0.16$ & $0.44 \pm 0.16$ & $0.59 \pm 0.15$ \\
\hline EPL1 & ----- & ----- & ----- & EPL1 & $0.57 \pm 0.17$ & $0.48 \pm 0.13$ & $0.54 \pm 0.17$ \\
\hline EPL2 & ----- & ----- & ----- & EPL2 & $0.58 \pm 0.16$ & $0.49 \pm 0.13$ & $0.54 \pm 0.16$ \\
\hline LPE & ----- & ----- & ----- & LPE & $0.56 \pm 0.11$ & $0.54 \pm 0.12$ & $0.50 \pm 0.11$ \\
\hline BSM & $0.49 \pm 0.17$ & $0.45 \pm 0.13$ & $0.41 \pm 0.12$ & BSM & $0.64 \pm 0.14$ & $0.50 \pm 0.09$ & $0.61 \pm 0.10$ \\
\hline LSM & $0.53 \pm 0.20$ & $0.48 \pm 0.14$ & $0.49 \pm 0.14$ & LSM & $0.66 \pm 0.17$ & $0.55 \pm 0.12$ & $0.58 \pm 0.15$ \\
\hline PSM & $0.51 \pm 0.17$ & $0.43 \pm 0.13$ & $0.46 \pm 0.18$ & PSM & $0.63 \pm 0.18$ & $0.54 \pm 0.14$ & $0.55 \pm 0.12$ \\
\hline HLM & ----- & $\begin{array}{l}---- \\
\end{array}$ & ----- & HLM & $0.58 \pm 0.20$ & $0.61 \pm 0.12$ & $0.57 \pm 0.11$ \\
\hline HPM & $\begin{array}{l}---- \\
--1\end{array}$ & $\begin{array}{l}---- \\
---\end{array}$ & $\begin{array}{l}---- \\
-\cdots\end{array}$ & HPM & $0.67 \pm 0.13$ & $0.57 \pm 0.15$ & $0.51 \pm 0.12$ \\
\hline POPI & ----- & ----- & ----- & POPI & $0.65 \pm 0.16$ & $0.56 \pm 0.15$ & $0.57 \pm 0.15$ \\
\hline SAPI & ----- & ----- & ----- & SAPI & $0.68 \pm 0.15$ & $0.61 \pm 0.15$ & $0.60 \pm 0.15$ \\
\hline DPPS & ----- & ----- & ----- & DPPS & $0.58 \pm 0.16$ & $0.54 \pm 0.13$ & $0.56 \pm 0.15$ \\
\hline POPS & ----- & $\begin{array}{ll}---- \\
\end{array}$ & $\begin{array}{ll}---- \\
\end{array}$ & POPS & $0.61 \pm 0.17$ & $0.46 \pm 0.14$ & $0.54 \pm 0.14$ \\
\hline DOPS & ----- & ----- & ----- & DOPS & $0.60 \pm 0.16$ & $0.50 \pm 0.13$ & $0.57 \pm 0.14$ \\
\hline DSPS & ----- & ----- & ----- & DSPS & $0.61 \pm 0.16$ & $0.52 \pm 0.15$ & $0.56 \pm 0.14$ \\
\hline DPPG & ----- & ----- & ----- & DPPG & $0.67 \pm 0.14$ & $0.52 \pm 0.11$ & $0.66 \pm 0.14$ \\
\hline DOPG & $\begin{array}{ll}---- \\
-\end{array}$ & $\begin{array}{ll}---- \\
\end{array}$ & $\begin{array}{ll}---- \\
\end{array}$ & DOPG & $0.67 \pm 0.17$ & $0.53 \pm 0.12$ & $0.56 \pm 0.17$ \\
\hline DPPA & ----- & ----- & ----- & DPPA & $0.60 \pm 0.19$ & $0.55 \pm 0.13$ & $0.50 \pm 0.13$ \\
\hline POPA & $\begin{array}{ll}---- \\
\end{array}$ & ----- & ----- & POPA & $0.62 \pm 0.17$ & $0.51 \pm 0.15$ & $0.51 \pm 0.16$ \\
\hline
\end{tabular}


Table S3. Average values (with standard deviations) of the area per lipid in the outer and in the inner monolayer of the membrane in the NPT-iso, NPT-semi, and NP $\gamma \mathrm{T}$ simulations in the 650750 ns trajectory segment

\begin{tabular}{|c|c|c|c|c|c|c|c|}
\hline \multicolumn{4}{|c|}{ Outer monolayer } & \multicolumn{4}{|c|}{ Inner monolayer } \\
\hline & NPT-iso & NPT-semi & $\mathbf{N P} \gamma \mathbf{T}$ & & NPT-iso & NPT-semi & $\mathbf{N P} \gamma \mathbf{T}$ \\
\hline Lipid & Area $\left[\mathrm{nm}^{2}\right]$ & Area $\left[\mathrm{nm}^{2}\right]$ & Area $\left[\mathrm{nm}^{2}\right]$ & Lipid & Area $\left[\mathrm{nm}^{2}\right]$ & Area $\left[\mathrm{nm}^{2}\right]$ & Area $\left[\mathrm{nm}^{2}\right]$ \\
\hline CHL & $0.44 \pm 0.15$ & $0.39 \pm 0.12$ & $0.42 \pm 0.13$ & CHL & ----- & ----- & ----- \\
\hline DMPC & $0.63 \pm 0.19$ & $0.47 \pm 0.12$ & $0.47 \pm 0.12$ & DMPC & $0.65 \pm 0.24$ & $0.52 \pm 0.12$ & $0.52 \pm 0.17$ \\
\hline DPPC & $0.57 \pm 0.19$ & $0.46 \pm 0.14$ & $0.56 \pm 0.17$ & DPPC & $0.65 \pm 0.15$ & $0.59 \pm 0.12$ & $0.63 \pm 0.20$ \\
\hline POPC & $0.62 \pm 0.21$ & $0.50 \pm 0.15$ & $0.54 \pm 0.18$ & POPC & $0.66 \pm 0.17$ & $0.56 \pm 0.13$ & $0.57 \pm 0.18$ \\
\hline DOPC & $0.64 \pm 0.20$ & $0.53 \pm 0.15$ & $0.55 \pm 0.18$ & DOPC & $0.65 \pm 0.18$ & $0.56 \pm 0.12$ & $0.59 \pm 0.16$ \\
\hline SOPC & $0.65 \pm 0.21$ & $0.56 \pm 0.13$ & $0.63 \pm 0.17$ & SOPC & ----- & ----- & ----- \\
\hline AOPC & ----- & ----- & ----- & AOPC & $0.62 \pm 0.13$ & $0.52 \pm 0.12$ & $0.61 \pm 0.11$ \\
\hline SAPC & $0.71 \pm 0.20$ & $0.55 \pm 0.14$ & $0.57 \pm 0.20$ & SAPC & $0.70 \pm 0.18$ & $0.59 \pm 0.12$ & $0.61 \pm 0.12$ \\
\hline APPC & $\begin{array}{ll}---- \\
\end{array}$ & $\begin{array}{ll}---- \\
\end{array}$ & $\begin{array}{ll}---- \\
\end{array}$ & APPC & $0.66 \pm 0.14$ & $0.45 \pm 0.10$ & $0.54 \pm 0.13$ \\
\hline ASPC & $\begin{array}{ll}---- \\
\end{array}$ & ----- & ----- & ASPC & $0.71 \pm 0.14$ & $0.59 \pm 0.10$ & $0.67 \pm 0.15$ \\
\hline LPC & $0.52 \pm 0.16$ & $0.41 \pm 0.12$ & $0.53 \pm 0.23$ & LPC & ----- & ----- & $\begin{array}{l}---- \\
\end{array}$ \\
\hline SPC & $\begin{array}{l}---- \\
\end{array}$ & $\begin{array}{l}---- \\
\end{array}$ & $\begin{array}{l}---- \\
\end{array}$ & SPC & $0.51 \pm 0.11$ & $0.49 \pm 0.14$ & $0.41 \pm 0.14$ \\
\hline SOPE & $0.48 \pm 0.15$ & $0.47 \pm 0.13$ & $0.63 \pm 0.17$ & SOPE & $0.61 \pm 0.16$ & $0.47 \pm 0.18$ & $0.56 \pm 0.14$ \\
\hline SAPE & ----- & ----- & ----- & SAPE & $0.64 \pm 0.19$ & $0.48 \pm 0.13$ & $0.56 \pm 0.14$ \\
\hline DAPE & ----- & ----- & ----- & DAPE & $0.63 \pm 0.20$ & $0.50 \pm 0.11$ & $0.54 \pm 0.17$ \\
\hline SDPE & ----- & ----- & ----- & SDPE & $0.59 \pm 0.16$ & $0.57 \pm 0.15$ & $0.50 \pm 0.14$ \\
\hline DGPE & ----- & ----- & ----- & DGPE & $0.60 \pm 0.16$ & $0.53 \pm 0.14$ & $0.53 \pm 0.14$ \\
\hline EPL1 & ----- & ----- & ----- & EPL1 & $0.57 \pm 0.17$ & $0.49 \pm 0.13$ & $0.55 \pm 0.19$ \\
\hline EPL2 & ----- & ----- & ----- & EPL2 & $0.61 \pm 0.16$ & $0.50 \pm 0.14$ & $0.54 \pm 0.15$ \\
\hline LPE & ----- & ----- & ----- & LPE & $0.60 \pm 0.11$ & $0.42 \pm 0.07$ & $0.51 \pm 0.10$ \\
\hline BSM & $0.50 \pm 0.15$ & $0.48 \pm 0.17$ & $0.53 \pm 0.17$ & BSM & $0.66 \pm 0.14$ & $0.52 \pm 0.10$ & $0.62 \pm 0.10$ \\
\hline LSM & $0.54 \pm 0.20$ & $0.45 \pm 0.12$ & $0.50 \pm 0.15$ & LSM & $0.57 \pm 0.17$ & $0.55 \pm 0.10$ & $0.57 \pm 0.13$ \\
\hline PSM & $0.44 \pm 0.19$ & $0.45 \pm 0.14$ & $0.45 \pm 0.14$ & PSM & $0.63 \pm 0.18$ & $0.50 \pm 0.13$ & $0.56 \pm 0.12$ \\
\hline HLM & ----- & ----- & ----- & HLM & $0.68 \pm 0.20$ & $0.42 \pm 0.11$ & $0.55 \pm 0.09$ \\
\hline HPM & $\begin{array}{l}---- \\
\end{array}$ & $\begin{array}{l}---- \\
\end{array}$ & $\begin{array}{l}---- \\
\end{array}$ & HPM & $0.56 \pm 0.13$ & $0.50 \pm 0.08$ & $0.51 \pm 0.10$ \\
\hline POPI & ----- & ----- & ----- & POPI & $0.67 \pm 0.16$ & $0.52 \pm 0.13$ & $0.60 \pm 0.16$ \\
\hline SAPI & ----- & ----- & ----- & SAPI & $0.67 \pm 0.15$ & $0.57 \pm 0.12$ & $0.58 \pm 0.16$ \\
\hline DPPS & ----- & ----- & ----- & DPPS & $0.65 \pm 0.17$ & $0.55 \pm 0.14$ & $0.62 \pm 0.18$ \\
\hline POPS & ----- & $\begin{array}{l}---- \\
\end{array}$ & $\begin{array}{l}---- \\
\end{array}$ & POPS & $0.60 \pm 0.16$ & $0.51 \pm 0.14$ & $0.55 \pm 0.15$ \\
\hline DOPS & ----- & ----- & ----- & DOPS & $0.57 \pm 0.16$ & $0.52 \pm 0.11$ & $0.53 \pm 0.13$ \\
\hline DSPS & ----- & ----- & ----- & DSPS & $0.60 \pm 0.14$ & $0.55 \pm 0.20$ & $0.54 \pm 0.14$ \\
\hline DPPG & ----- & ----- & ----- & DPPG & $0.70 \pm 0.17$ & $0.55 \pm 0.14$ & $0.63 \pm 0.15$ \\
\hline DOPG & $\begin{array}{l}---- \\
\end{array}$ & $\begin{array}{l}---- \\
\end{array}$ & $\begin{array}{l}---- \\
\end{array}$ & DOPG & $0.67 \pm 0.19$ & $0.63 \pm 0.13$ & $0.58 \pm 0.13$ \\
\hline DPPA & ----- & ----- & ----- & DPPA & $0.59 \pm 0.16$ & $0.53 \pm 0.12$ & $0.51 \pm 0.11$ \\
\hline POPA & ----- & ----- & ----- & POPA & $0.63 \pm 0.16$ & $0.36 \pm 0.10$ & $0.60 \pm 0.23$ \\
\hline
\end{tabular}


Table S4. Average values (with standard deviations) of the area per lipid in the outer and in the inner monolayer of the membrane in the NPT-iso, NPT-semi, and NP $\gamma$ T simulations in the 9001000 ns trajectory segment

\begin{tabular}{|c|c|c|c|c|c|c|c|}
\hline \multicolumn{4}{|c|}{ Outer monolayer } & \multicolumn{4}{|c|}{ Inner monolayer } \\
\hline & NPT-iso & NPT-semi & $\mathbf{N P} \gamma \mathbf{T}$ & & NPT-iso & NPT-semi & $\mathbf{N P} \gamma \mathbf{T}$ \\
\hline Lipid & Area $\left[\mathrm{nm}^{2}\right]$ & Area $\left[\mathrm{nm}^{2}\right]$ & Area $\left[\mathrm{nm}^{2}\right]$ & Lipid & Area $\left[\mathrm{nm}^{2}\right]$ & Area $\left[\mathrm{nm}^{2}\right]$ & Area $\left[\mathrm{nm}^{2}\right]$ \\
\hline $\mathrm{CHL}$ & $0.43 \pm 0.16$ & $0.40 \pm 0.12$ & $0.41 \pm 0.14$ & $\mathrm{CHL}$ & ----- & ----- & ----- \\
\hline DMPC & $0.73 \pm 0.22$ & $0.45 \pm 0.12$ & $0.49 \pm 0.12$ & DMPC & $0.67 \pm 0.15$ & $0.60 \pm 0.17$ & $0.60 \pm 0.13$ \\
\hline DPPC & $0.54 \pm 0.18$ & $0.48 \pm 0.14$ & $0.59 \pm 0.20$ & DPPC & $0.68 \pm 0.17$ & $0.55 \pm 0.14$ & $0.60 \pm 0.14$ \\
\hline POPC & $0.60 \pm 0.19$ & $0.50 \pm 0.14$ & $0.55 \pm 0.23$ & POPC & $0.67 \pm 0.17$ & $0.57 \pm 0.15$ & $0.62 \pm 0.15$ \\
\hline DOPC & $0.64 \pm 0.24$ & $0.51 \pm 0.15$ & $0.61 \pm 0.24$ & DOPC & $0.66 \pm 0.17$ & $0.57 \pm 0.14$ & $0.60 \pm 0.18$ \\
\hline SOPC & $0.63 \pm 0.23$ & $0.55 \pm 0.17$ & $0.53 \pm 0.16$ & SOPC & ----- & ----- & ----- \\
\hline AOPC & $\begin{array}{ll}---- \\
--\end{array}$ & $\begin{array}{ll}---- \\
--\end{array}$ & $\begin{array}{ll}---- \\
--\end{array}$ & AOPC & $0.74 \pm 0.14$ & $0.49 \pm 0.08$ & $0.63 \pm 0.12$ \\
\hline SAPC & $0.66 \pm 0.21$ & $0.56 \pm 0.14$ & $0.53 \pm 0.27$ & SAPC & $0.72 \pm 0.15$ & $0.60 \pm 0.13$ & $0.62 \pm 0.13$ \\
\hline APPC & $\begin{array}{ll}---- \\
\end{array}$ & $\begin{array}{ll}---- \\
\end{array}$ & $\begin{array}{ll}---- \\
\end{array}$ & APPC & $0.72 \pm 0.15$ & $0.60 \pm 0.10$ & $0.60 \pm 0.10$ \\
\hline ASPC & $\begin{array}{ll}---- \\
\end{array}$ & $\begin{array}{ll}---- \\
\end{array}$ & $\begin{array}{ll}---- \\
\end{array}$ & ASPC & $0.67 \pm 0.14$ & $0.61 \pm 0.14$ & $0.70 \pm 0.13$ \\
\hline LPC & $0.46 \pm 0.18$ & $0.42 \pm 0.15$ & $0.49 \pm 0.20$ & LPC & $\begin{array}{ll}---- \\
\end{array}$ & ----- & ----- \\
\hline SPC & $\begin{array}{ll}---- \\
-\end{array}$ & $\begin{array}{ll}---- \\
\end{array}$ & $\begin{array}{ll}---- \\
\end{array}$ & SPC & $0.52 \pm 0.12$ & $0.39 \pm 0.07$ & $0.53 \pm 0.10$ \\
\hline SOPE & $0.53 \pm 0.14$ & $0.48 \pm 0.11$ & $0.56 \pm 0.16$ & SOPE & $0.62 \pm 0.19$ & $0.53 \pm 0.15$ & $0.55 \pm 0.16$ \\
\hline SAPE & ----- & ----- & ----- & SAPE & $0.61 \pm 0.15$ & $0.49 \pm 0.14$ & $0.56 \pm 0.13$ \\
\hline DAPE & $\begin{array}{ll}---- \\
\end{array}$ & $\begin{array}{ll}---- \\
\end{array}$ & $\begin{array}{ll}---- \\
\end{array}$ & DAPE & $0.60 \pm 0.16$ & $0.50 \pm 0.12$ & $0.58 \pm 0.17$ \\
\hline SDPE & $\begin{array}{ll}---- \\
--\end{array}$ & $\begin{array}{ll}---- \\
-\end{array}$ & $\begin{array}{ll}---- \\
\end{array}$ & SDPE & $0.64 \pm 0.19$ & $0.48 \pm 0.15$ & $0.55 \pm 0.15$ \\
\hline DGPE & $\begin{array}{l}---- \\
\end{array}$ & $\begin{array}{l}---- \\
\end{array}$ & $\begin{array}{l}---- \\
\end{array}$ & DGPE & $0.58 \pm 0.15$ & $0.49 \pm 0.11$ & $0.50 \pm 0.16$ \\
\hline EPL1 & ----- & ----- & ----- & EPL1 & $0.60 \pm 0.17$ & $0.50 \pm 0.12$ & $0.52 \pm 0.16$ \\
\hline EPL2 & ----- & ----- & ----- & EPL2 & $0.56 \pm 0.16$ & $0.51 \pm 0.13$ & $0.52 \pm 0.16$ \\
\hline LPE & ----- & ----- & ----- & LPE & $0.65 \pm 0.16$ & $0.49 \pm 0.08$ & $0.47 \pm 0.12$ \\
\hline $\mathrm{BSM}$ & $0.46 \pm 0.14$ & $0.47 \pm 0.11$ & $0.49 \pm 0.20$ & $\mathrm{BSM}$ & $0.66 \pm 0.15$ & $0.52 \pm 0.09$ & $0.53 \pm 0.17$ \\
\hline LSM & $0.49 \pm 0.20$ & $0.45 \pm 0.13$ & $0.45 \pm 0.14$ & LSM & $0.58 \pm 0.14$ & $0.52 \pm 0.11$ & $0.51 \pm 0.14$ \\
\hline PSM & $0.47 \pm 0.17$ & $0.46 \pm 0.13$ & $0.45 \pm 0.17$ & PSM & $0.59 \pm 0.16$ & $0.57 \pm 0.19$ & $0.59 \pm 0.21$ \\
\hline HLM & $\begin{array}{l}---- \\
\end{array}$ & $\begin{array}{l}---- \\
\end{array}$ & $\begin{array}{l}---- \\
\end{array}$ & HLM & $0.70 \pm 0.16$ & $0.55 \pm 0.12$ & $0.57 \pm 0.10$ \\
\hline HPM & $\begin{array}{l}---- \\
--\end{array}$ & $\begin{array}{l}---- \\
--\end{array}$ & $\begin{array}{l}---- \\
--\end{array}$ & HPM & $0.57 \pm 0.12$ & $0.54 \pm 0.09$ & $0.61 \pm 0.11$ \\
\hline POPI & ----- & ----- & ----- & POPI & $0.64 \pm 0.16$ & $0.54 \pm 0.13$ & $0.61 \pm 0.17$ \\
\hline SAPI & ----- & ----- & ----- & SAPI & $0.64 \pm 0.17$ & $0.56 \pm 0.13$ & $0.63 \pm 0.16$ \\
\hline DPPS & ----- & ----- & ----- & DPPS & $0.62 \pm 0.16$ & $0.52 \pm 0.14$ & $0.58 \pm 0.14$ \\
\hline POPS & ----- & $\begin{array}{ll}---- \\
\end{array}$ & $\begin{array}{ll}---- \\
\end{array}$ & POPS & $0.64 \pm 0.14$ & $0.49 \pm 0.13$ & $0.54 \pm 0.14$ \\
\hline DOPS & ----- & ----- & ----- & DOPS & $0.60 \pm 0.19$ & $0.55 \pm 0.18$ & $0.58 \pm 0.14$ \\
\hline DSPS & ----- & ----- & ----- & DSPS & $0.60 \pm 0.16$ & $0.50 \pm 0.13$ & $0.54 \pm 0.15$ \\
\hline DPPG & ----- & ----- & ----- & DPPG & $0.90 \pm 0.32$ & $0.57 \pm 0.11$ & $0.59 \pm 0.16$ \\
\hline DOPG & $\begin{array}{ll}---- \\
\end{array}$ & $\begin{array}{ll}---- \\
\end{array}$ & $\begin{array}{ll}---- \\
\end{array}$ & DOPG & $0.63 \pm 0.15$ & $0.64 \pm 0.11$ & $0.60 \pm 0.15$ \\
\hline DPPA & ----- & ----- & ----- & DPPA & $0.60 \pm 0.19$ & $0.50 \pm 0.15$ & $0.58 \pm 0.12$ \\
\hline POPA & $\begin{array}{l}---- \\
\end{array}$ & ----- & ----- & POPA & $0.62 \pm 0.16$ & $0.47 \pm 0.12$ & $0.58 \pm 0.26$ \\
\hline
\end{tabular}


Table S5. Type and duration of the flip-flop-like transitions of lipids between the outer and the inner monolayer of the membrane in the four trajectory segments of the simulations in NPT-iso and $\mathrm{NP} \gamma \mathrm{T}^{\#}$

\begin{tabular}{|c|c|c|c|c|}
\hline Ensemble & Time period & $\begin{array}{c}\text { Original } \\
\text { monolayer }\end{array}$ & Lipid & $\begin{array}{c}\text { Transition } \\
\text { duration [ns] }\end{array}$ \\
\hline \multirow{7}{*}{ NPT-iso } & $150-250[\mathrm{~ns}]$ & Outer & $\mathrm{CHL}$ & 0.20 \\
\hline & $650-750[\mathrm{~ns}]$ & Outer & CHL & 0.04 \\
\hline & \multirow{5}{*}{$900-1000[\mathrm{~ns}]$} & \multirow{2}{*}{ Outer } & CHL & 0.04 \\
\hline & & & LPC & 0.16 \\
\hline & & \multirow{3}{*}{ Inner } & LPE & 0.31 \\
\hline & & & SDPE & 0.24 \\
\hline & & & SAPE & 0.03 \\
\hline \multirow{10}{*}{$\mathbf{N P} \gamma \mathbf{T}$} & \multirow{3}{*}{$150-250[\mathrm{~ns}]$} & Outer & $\mathrm{CHL}$ & 27.54 \\
\hline & & \multirow{2}{*}{ Inner } & EPL2 & 0.03 \\
\hline & & & DPPG & 0.03 \\
\hline & \multirow{2}{*}{$450-550[\mathrm{~ns}]$} & Outer & $\mathrm{CHL}$ & 100.00 \\
\hline & & Inner & DAPE & 0.01 \\
\hline & \multirow{2}{*}{$650-750[\mathrm{~ns}]$} & Outer & CHL & 100.00 \\
\hline & & Inner & SPC & 0.03 \\
\hline & \multirow{3}{*}{$900-1000[\mathrm{~ns}]$} & Outer & $\mathrm{CHL}$ & $100.33^{\&}$ \\
\hline & & \multirow{2}{*}{ Inner } & DOPG & 0.05 \\
\hline & & & EPL2 & 0.01 \\
\hline
\end{tabular}

\#No such transitions are witnessed in NPT-semi

${ }^{\&}$ The excess 0.33 ns come from a second CHL molecule 

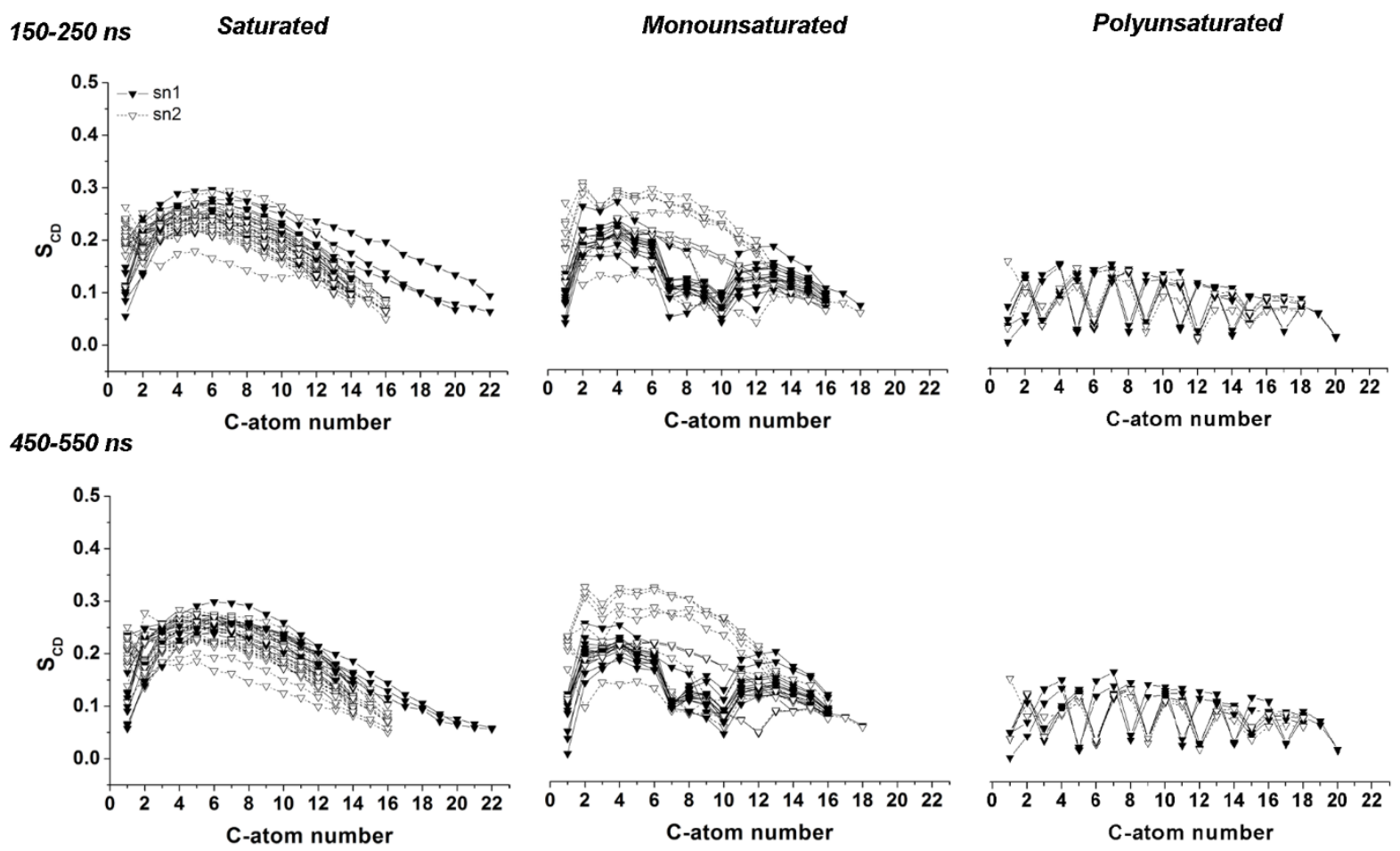

$650-750 n s$
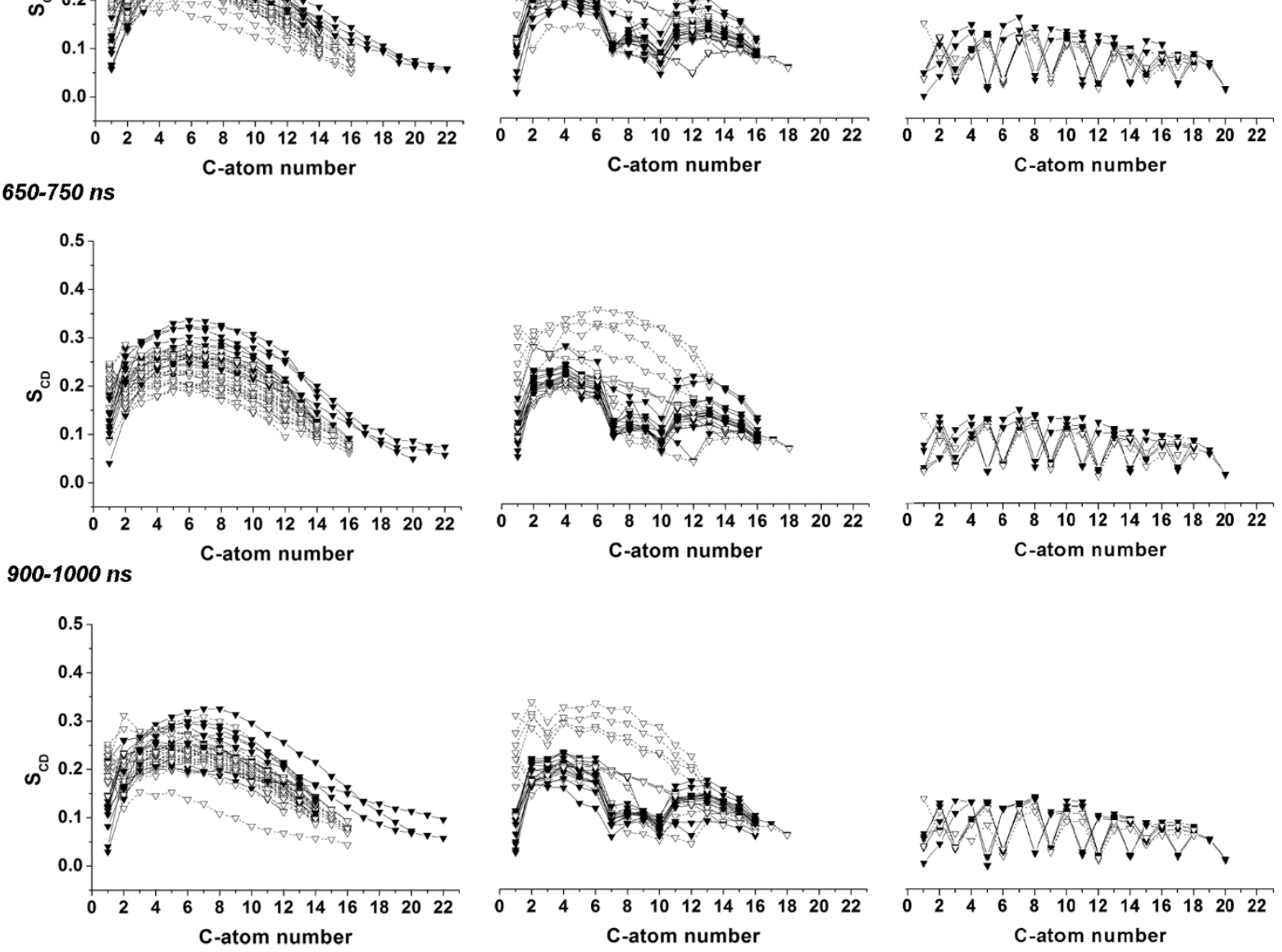

Figure S6. Deuterium order parameters of the carbon atoms from the lipid tails in the four trajectory segments of the NPT-iso simulation; snl tails are depicted by filled triangles connected by solid line; sn2 and single tails are depicted by empty triangles connected by dashed line; smaller numbers denote atoms closer to the head 

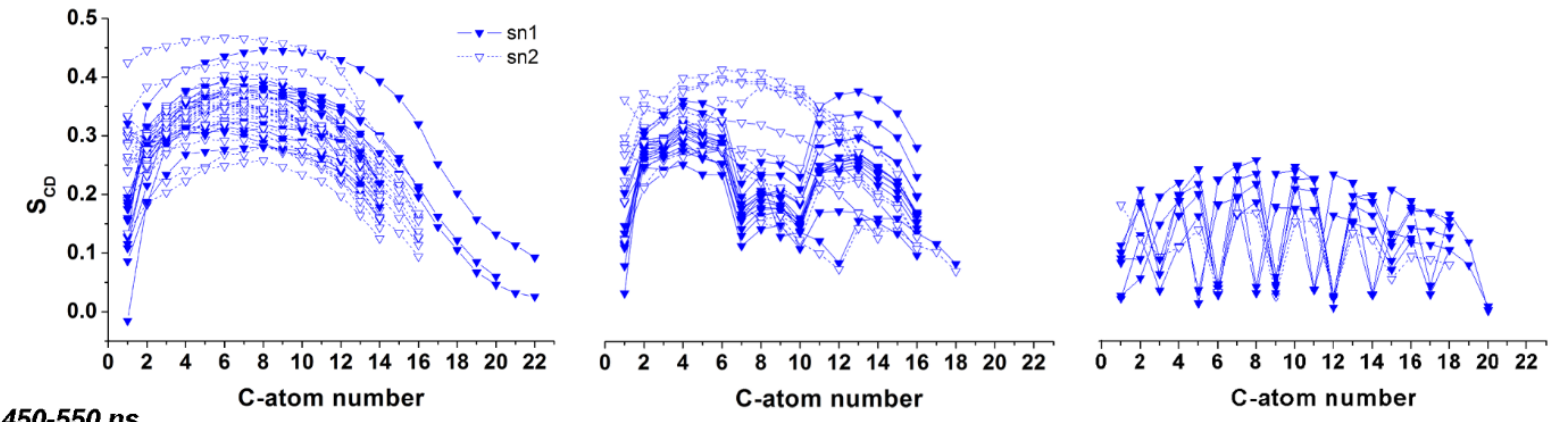

$450-550$ ns

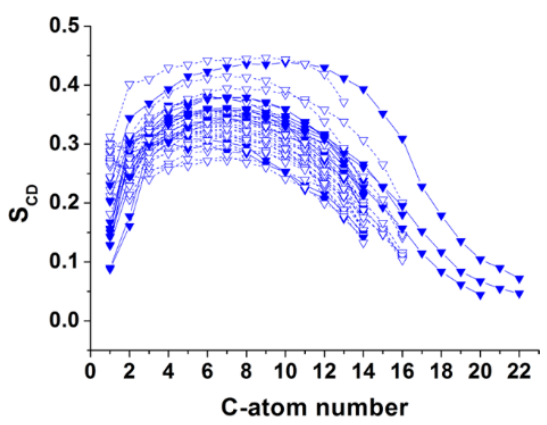

650-750 ns
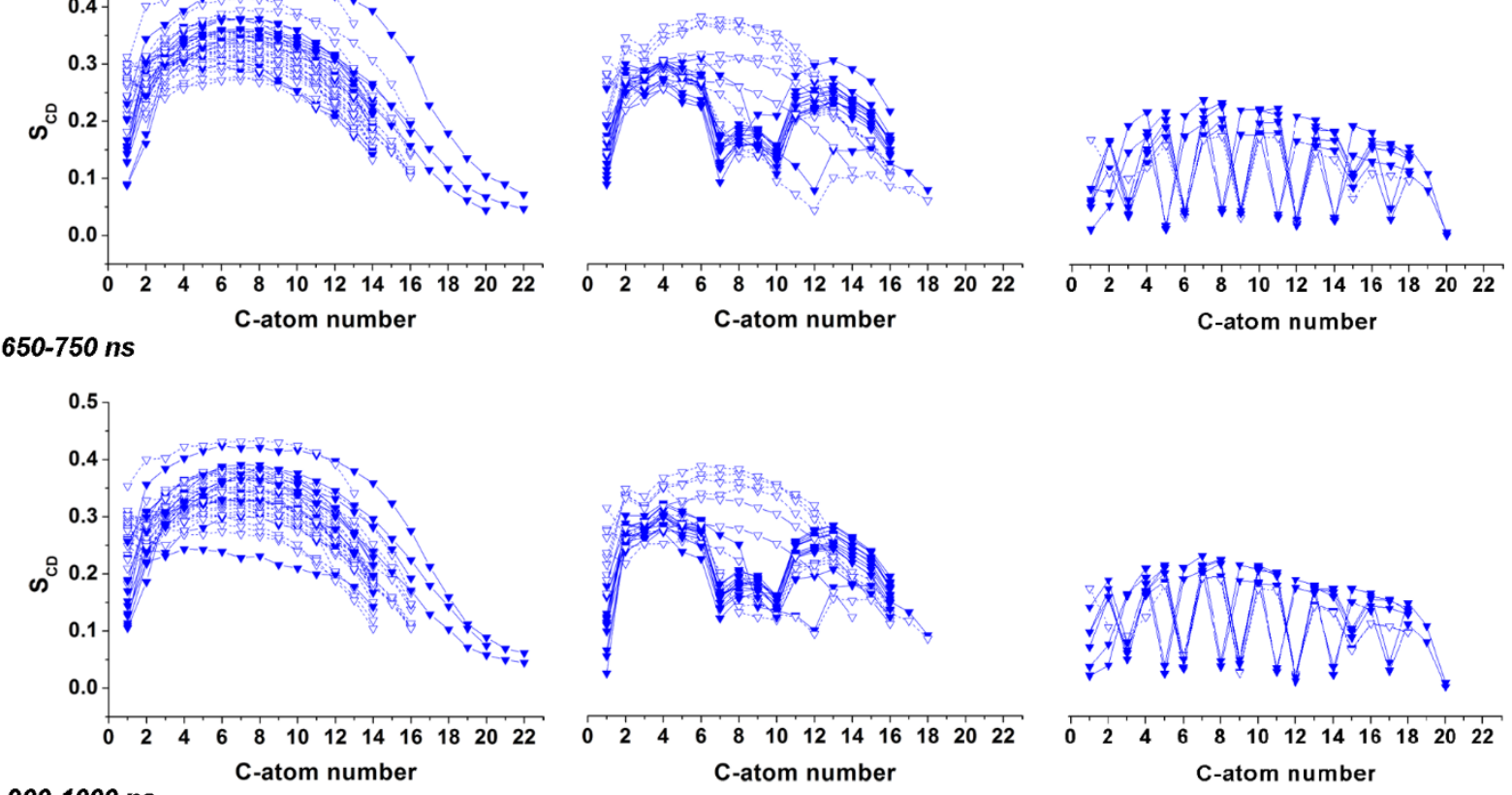

900-1000 ns
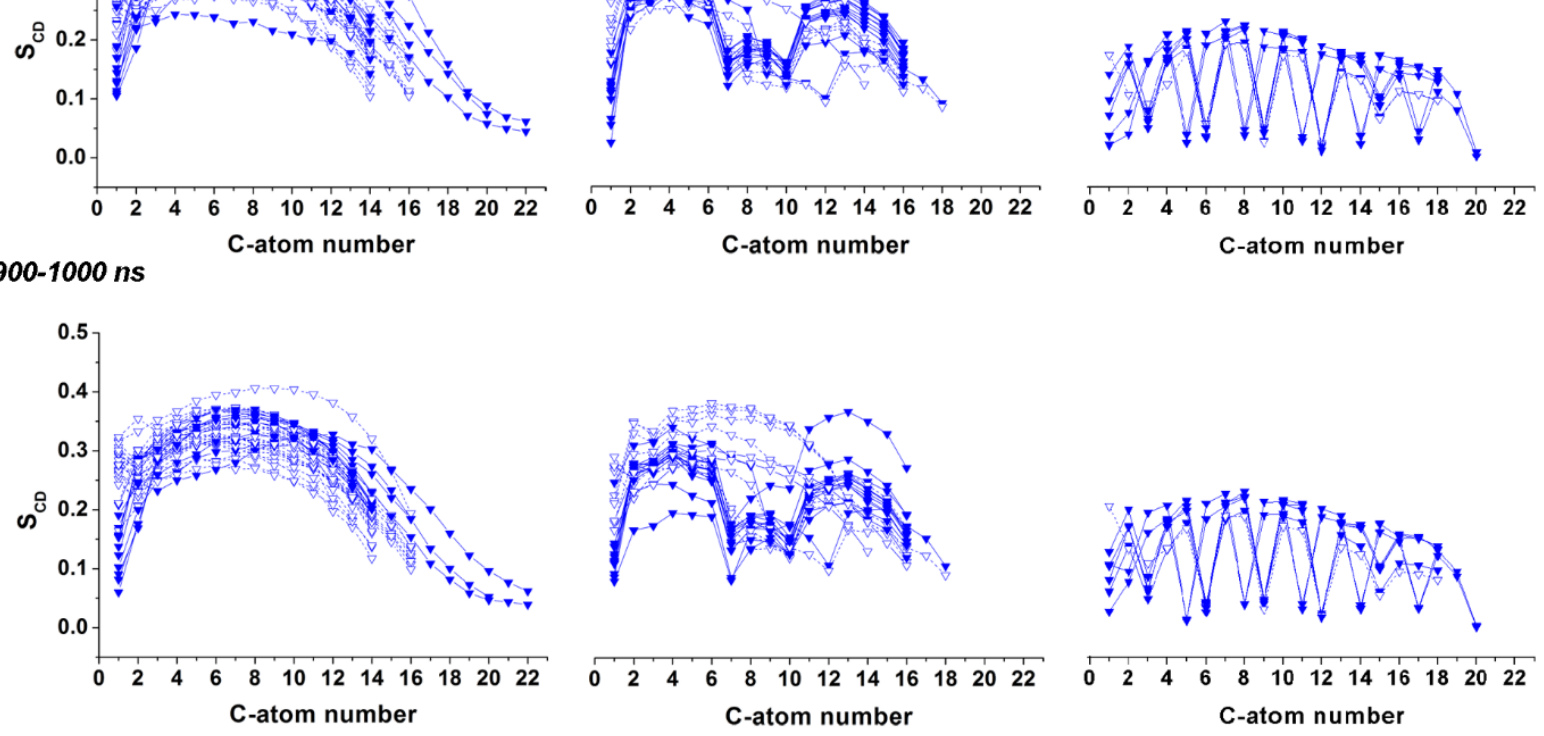

Figure S7. Deuterium order parameters of the carbon atoms from the lipid tails in the four trajectory segments of the NPT-semi simulation; snl tails are depicted by filled triangles connected by solid line; sn2 and single tails are depicted by empty triangles connected by dashed line; smaller numbers denote atoms closer to the head 

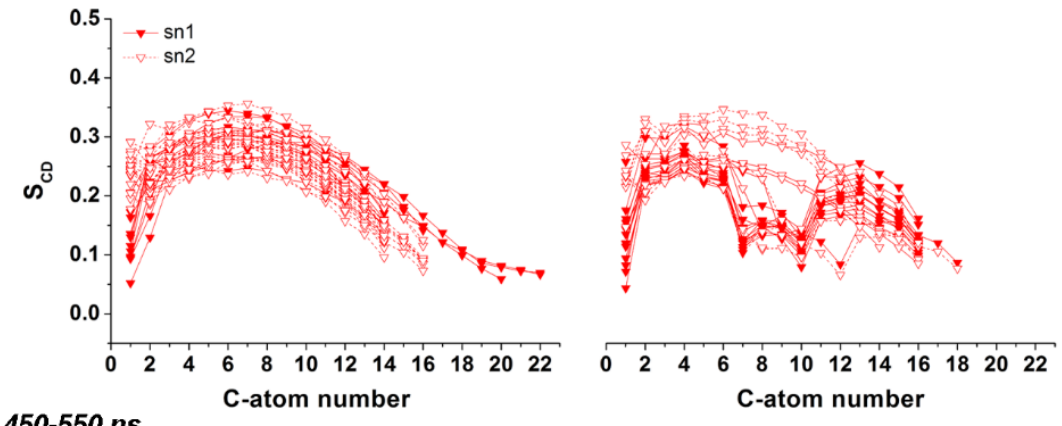

$450-550$ ns
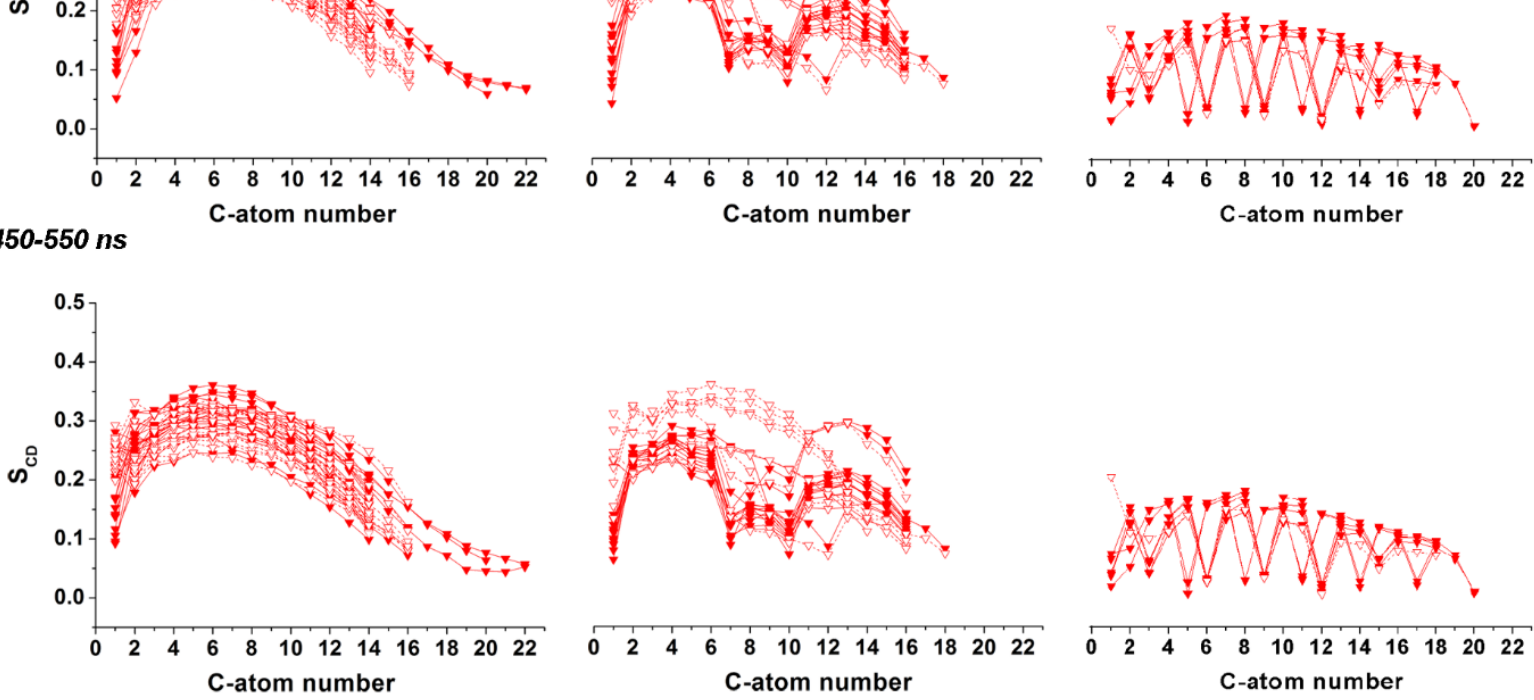

$650-750$ ns
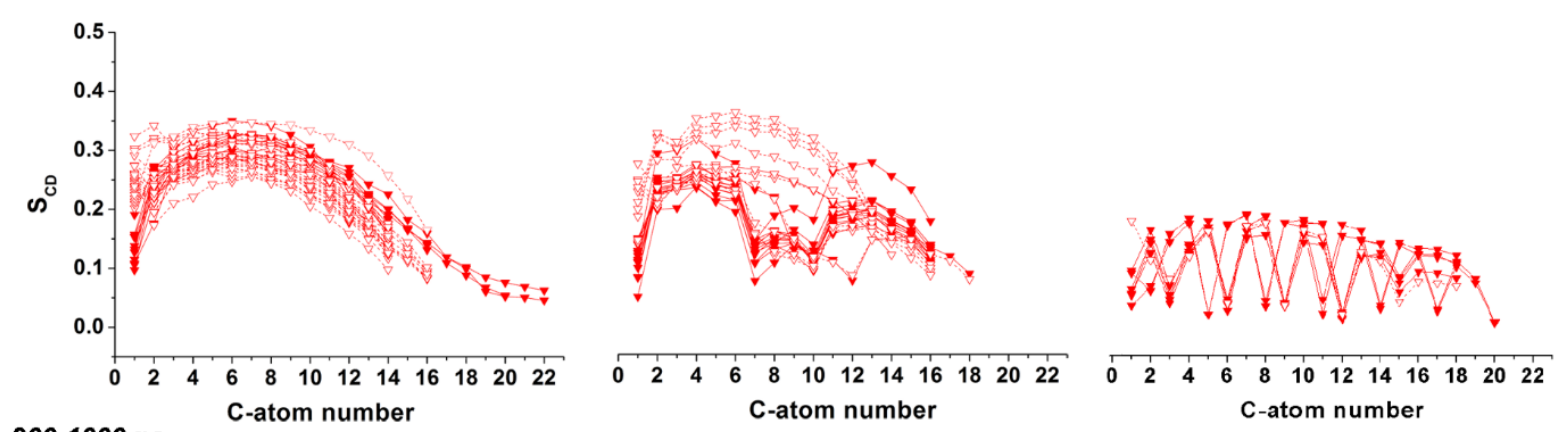

900-1000 ns
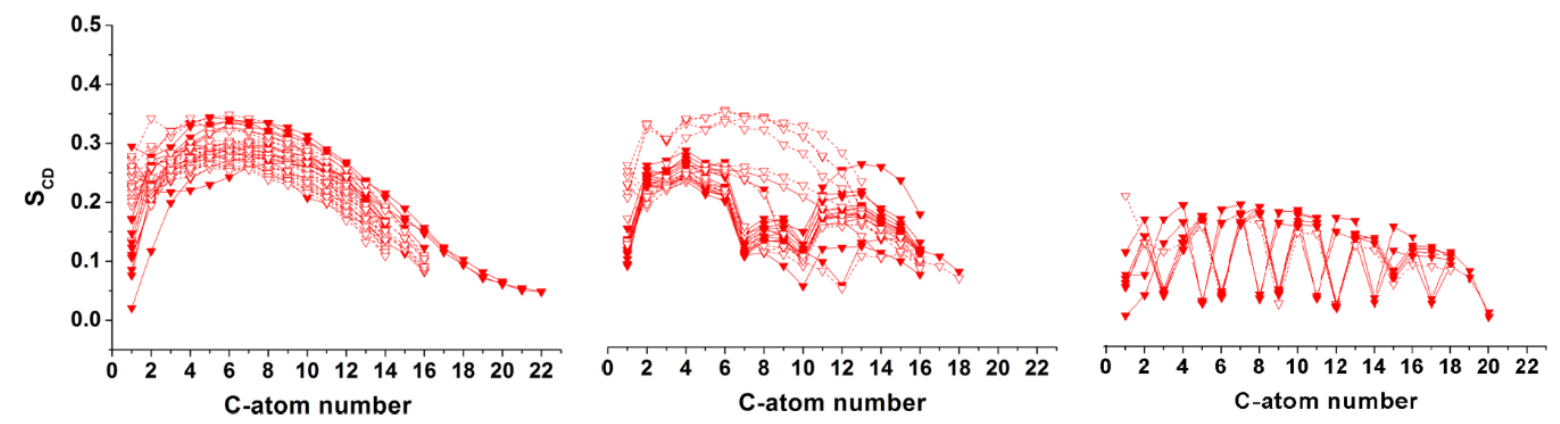

Figure S8. Deuterium order parameters of the carbon atoms from the lipid tails in the four trajectory segments of the NP $\gamma \mathrm{T}$ simulation; sn 1 tails are depicted by filled triangles connected by solid line; sn2 and single tails are depicted by empty triangles connected by dashed line; smaller numbers denote atoms closer to the head 
Distinct variation of $\mathrm{S}_{\mathrm{CD}}$ with time is observed in each simulation. There is slight increase of the order parameters of the saturated lipid tails from the membrane in NPT-iso after $650 \mathrm{~ns}$, suggesting more strict alignment. A similar change takes place for the monounsaturated tails earlier (after $450 \mathrm{~ns}$ ) but in the last TP some of them return to their previous state. The specific behavior of the tails with a double bond located near the head group, like those of BSM, PSM, LSM and the single hydrocarbon chain of SPC, can be outlined. They are characterized with more precise arrangement than the other ones, which maintain lesser degree of order. Furthermore, the drop in the $\mathrm{S}_{\mathrm{CD}}$ values for the atoms adjacent to the double bond is smaller. This is expected because a rotation about the neighboring single bond is much harder when the double one is closer to the head and the conformational change requires relocation of almost the whole chain. The same is valid for the tail of the two plasmalogen lipids, although their $\mathrm{S}_{\mathrm{CD}}$ values are lower. This behavior is found in the other two trajectories, but it is most pronounced in NPT-iso (and maybe in NP $\gamma \mathrm{T}$ ), where in the last TP these tails are markedly distinct from the rest. The flexibility of the polyunsaturated tails, unchanged during the whole trajectory, is clearly illustrated in the plot (Figure S6).

The membrane in NPT-semi is notably different. All order parameters are higher than in NPT-iso, suggesting more enhanced degree of ordering of the lipid tails. In the final $100 \mathrm{~ns}$, fragment disorder is introduced among the saturated tails accompanied by a certain homogenization of the alignment. The latter effect is found for the monounsaturated tails in the second and third TP but in the last one the relatively uniform behavior of the tails is lost. There is minor lowering of the order parameters of PUFAs after $450 \mathrm{~ns}$ and unification among $\mathrm{S}_{\mathrm{CD}}$ of the different species. The more ordered state of lipid tails is in line with the maxima in the mass density profiles (Figure S3) and with the elevated thickness of the bilayer in this trajectory (Table 1 of the main manuscript). Comparing this behavior with the previously described conduct of lipids in DPPC and mixed membranes simulated in NPT-iso and NPT-semi [Ref 25 of the main manuscript], some similarities and some differences may be outlined. As in the former bilayer (but to a lesser extent), the tails in NPT-semi are more straightened and the membrane is more ordered. Unlike before, however, where the order persists until the end of the simulation, a transition to the liquid ordered state takes place in the current system. This may be attributed to the enhanced lipid heterogeneity of the current bilayer.

The constant surface tension, maintained in the NP $\gamma \mathrm{T}$ simulation, results into lesser degree of ordering of all lipid tails than in NPT-semi but the system is not as disordered as when isotropic pressure scaling is applied. In the trajectory obtained in $\mathrm{NP} \gamma \mathrm{T}$, there is more pronounced liquefying of the membrane core, evident from the close-to-zero values of $\mathrm{S}_{\mathrm{CD}}$ of the longest saturated tails. Also, the evolution of the behavior of the saturated and PUFA tails is negligible and only minor for the monounsaturated ones. The ordering is more homogenous than in NPT-semi and this is even more pronounced in the second half of the simulation. 


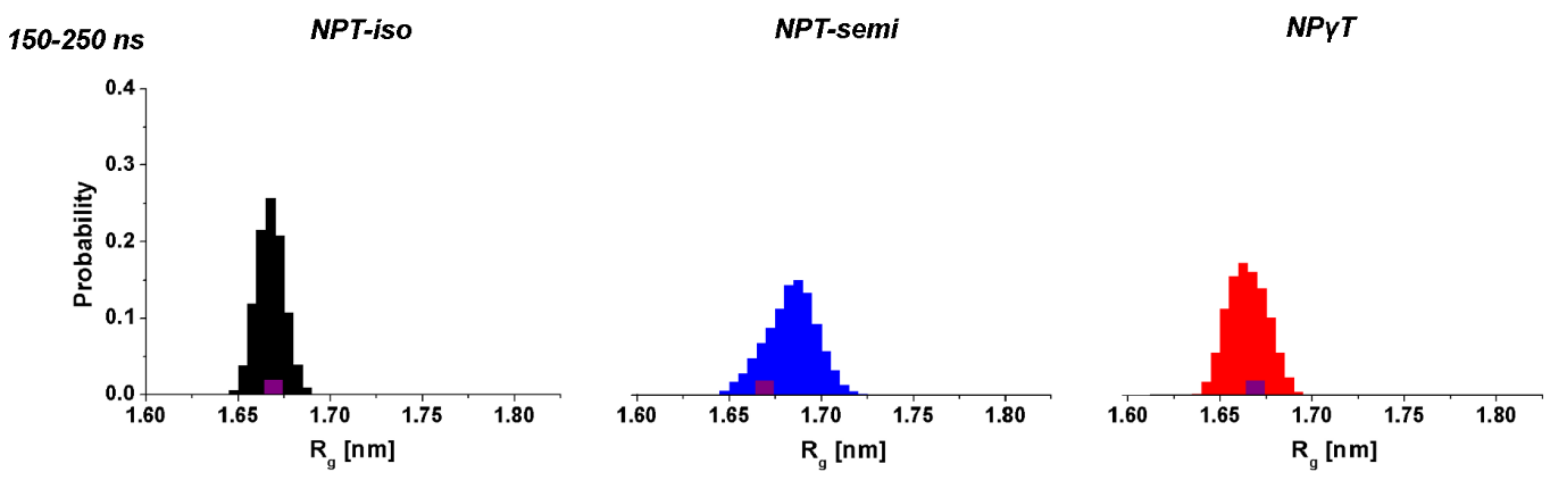

450-550 ns
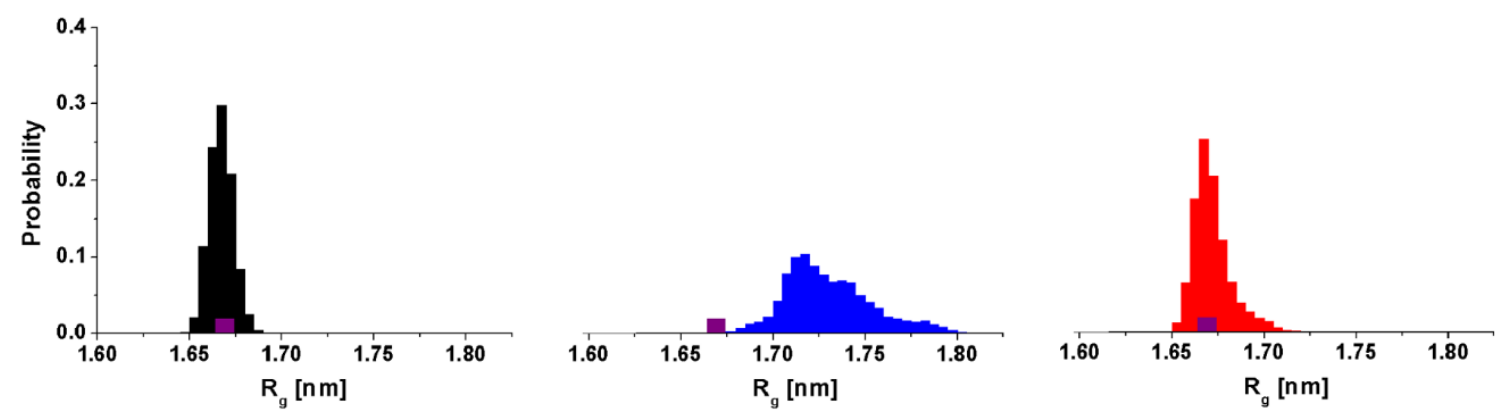

$650-750 \mathrm{~ns}$
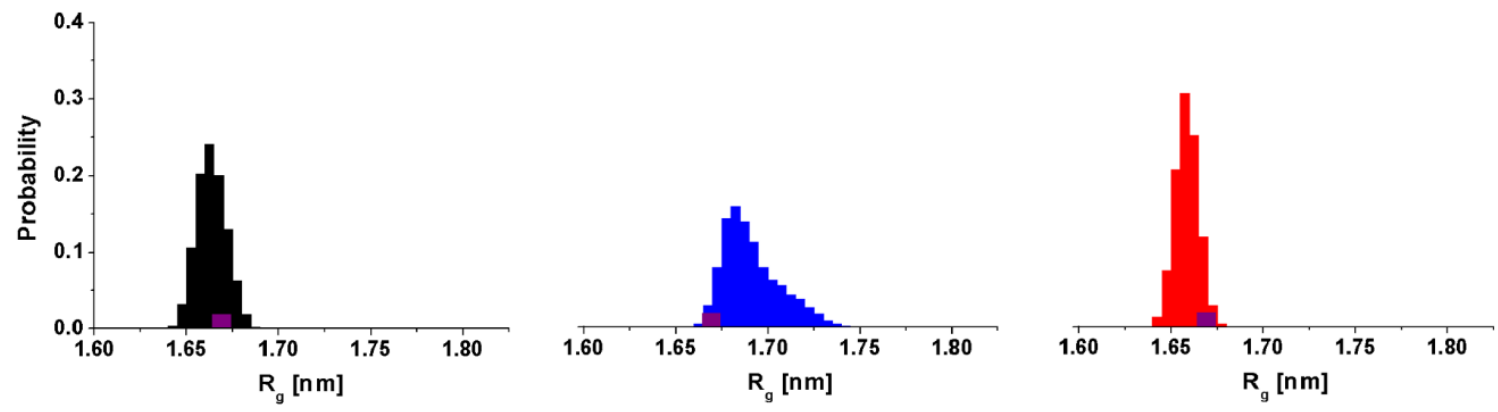

$900-1000 \mathrm{~ns}$
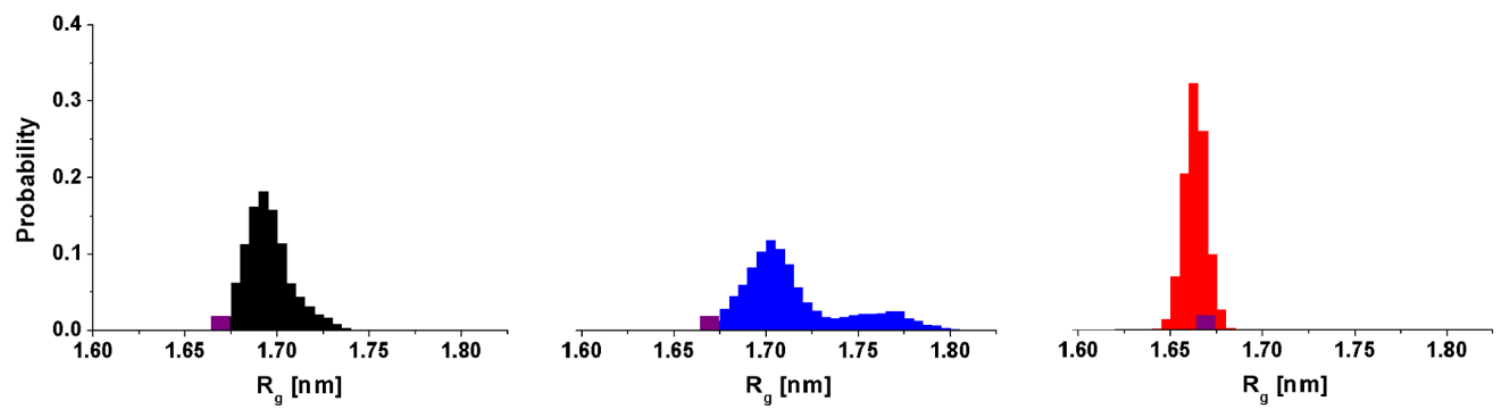

Figure S9. Distribution of the radius of gyration of the protein in the four trajectory segments of the NPT-iso (left), NPT-semi (middle), and NP $\gamma$ T (right) simulations; the radius of gyration of the experimental X-ray structure of $\mathrm{FR} \alpha$ is depicted as a purple square 

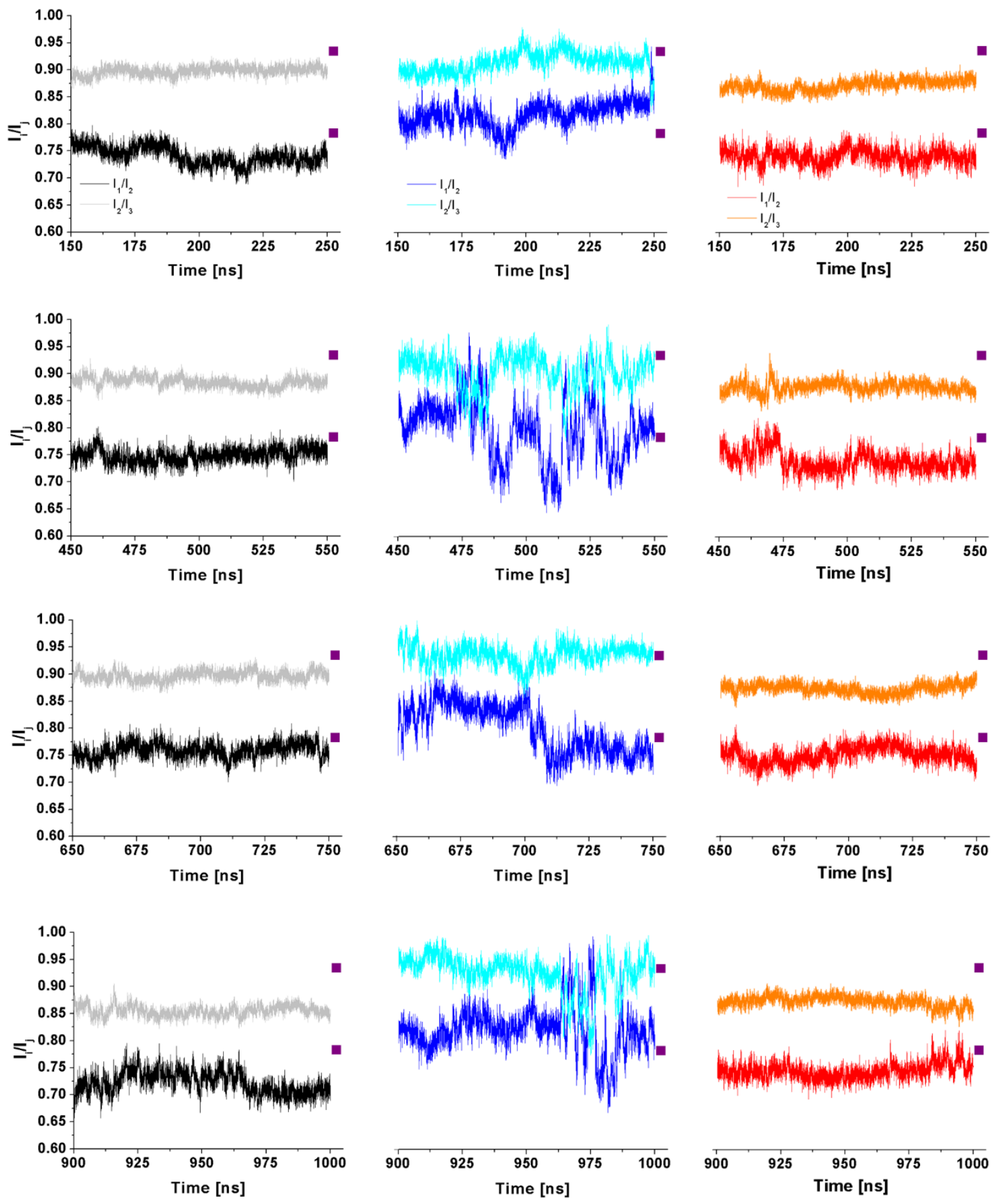

Figure S10. Evolution of the ratios of the principal moments of inertia of the protein in the four trajectory segments of the NPT-iso (left), NPT-semi (middle), and NPyT (right) simulations; the ratios for the experimental $\mathrm{X}$-ray structure of $\mathrm{FR} \alpha$ are depicted as purple squares 
Table S6. Average values (with standard deviations) of the radius of gyration and of the ratios of the principal moments of inertia of the protein in the four trajectory segments of the NPT-iso, NPT-semi, and NP $\gamma$ T simulations; the values for the X-ray structure are provided below the table for comparison

\begin{tabular}{|c|c|c|c|c|c|c|c|c|c|}
\hline & \multicolumn{3}{|c|}{ Radius of gyration [nm] } & \multicolumn{6}{|c|}{ Ratios $\mathbf{I}_{\mathbf{i}} / \mathbf{I}_{\mathbf{j}}$} \\
\hline \multirow[t]{2}{*}{ Time [ns] } & NPT(iso) & NPT(semi) & NP $\gamma \mathbf{T}$ & \multicolumn{2}{|c|}{ NPT(iso) } & \multicolumn{2}{|c|}{ NPT(semi) } & \multicolumn{2}{|c|}{$\mathbf{N P} \gamma \mathbf{T}$} \\
\hline & & & & $\mathbf{I}_{1} / \mathbf{I}_{2}$ & $\mathbf{I}_{2} / \mathbf{I}_{3}$ & $\mathbf{I}_{1} / \mathbf{I}_{2}$ & $\mathbf{I}_{2} / \mathbf{I}_{3}$ & $\mathbf{I}_{1} / \mathbf{I}_{2}$ & $\mathbf{I}_{2} / \mathbf{I}_{3}$ \\
\hline $150-250$ & $1.66 \pm 0.01$ & $1.68 \pm 0.01$ & $1.66 \pm 0.01$ & $0.74 \pm 0.02$ & $0.89 \pm 0.01$ & $0.82 \pm 0.02$ & $0.91 \pm 0.02$ & $0.74 \pm 0.01$ & $0.87 \pm 0.01$ \\
\hline 450-550 & $1.67 \pm 0.01$ & $1.73 \pm 0.02$ & $1.67 \pm 0.01$ & $0.75 \pm 0.01$ & $0.88 \pm 0.01$ & $0.80 \pm 0.06$ & $0.90 \pm 0.03$ & $0.74 \pm 0.02$ & $0.88 \pm 0.01$ \\
\hline $650-750$ & $1.66 \pm 0.01$ & $1.69 \pm 0.02$ & $1.66 \pm 0.01$ & $0.76 \pm 0.01$ & $0.90 \pm 0.01$ & $0.80 \pm 0.04$ & $0.94 \pm 0.02$ & $0.75 \pm 0.02$ & $0.87 \pm 0.01$ \\
\hline 900-1000 & $1.69 \pm 0.01$ & $\begin{array}{c}1.71 \pm 0.01^{\#} \\
1.76 \pm 0.02\end{array}$ & $1.66 \pm 0.01$ & $0.72 \pm 0.02$ & $0.85 \pm 0.01$ & $0.82 \pm 0.04$ & $0.93 \pm 0.03$ & $0.75 \pm 0.02$ & $0.87 \pm 0.01$ \\
\hline
\end{tabular}

$X$-ray: $R_{g}=1.67 \mathrm{~nm} ; \mathrm{I}_{1} / \mathrm{I}_{2}=0.78 ; \mathrm{I}_{2} / \mathrm{I}_{3}=0.93$

\#Separate averages are given for the two peaks of the distribution 
Root-mean-square fluctuations of the amino acids
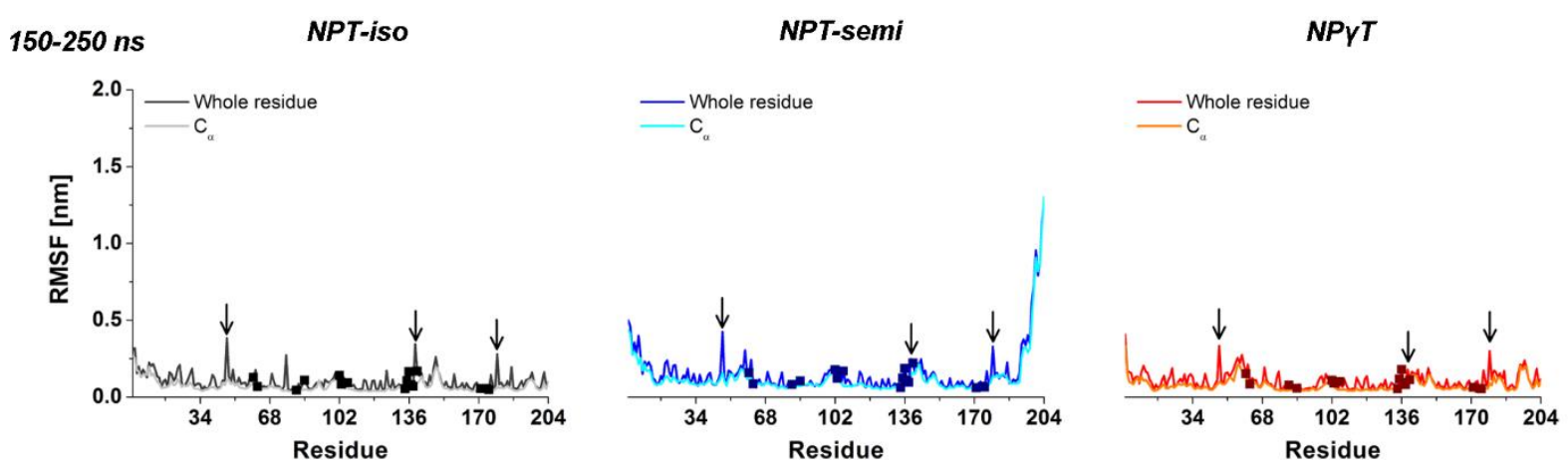

450-550 ns
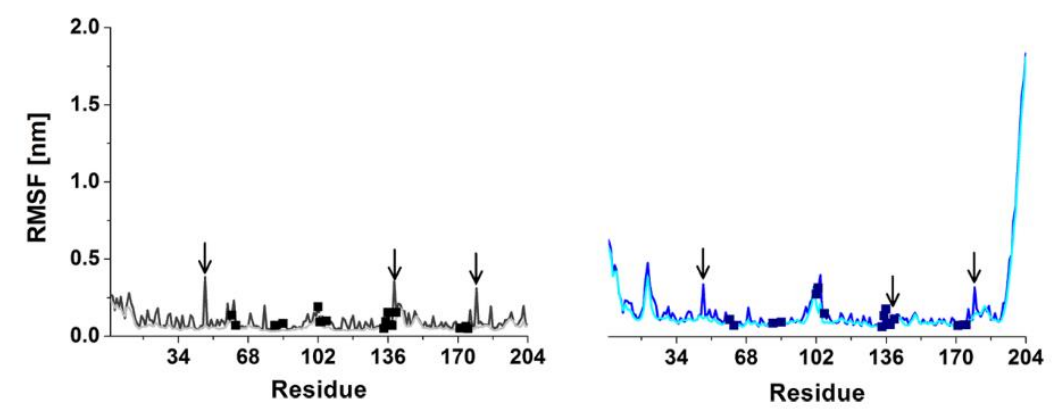

650-750 ns
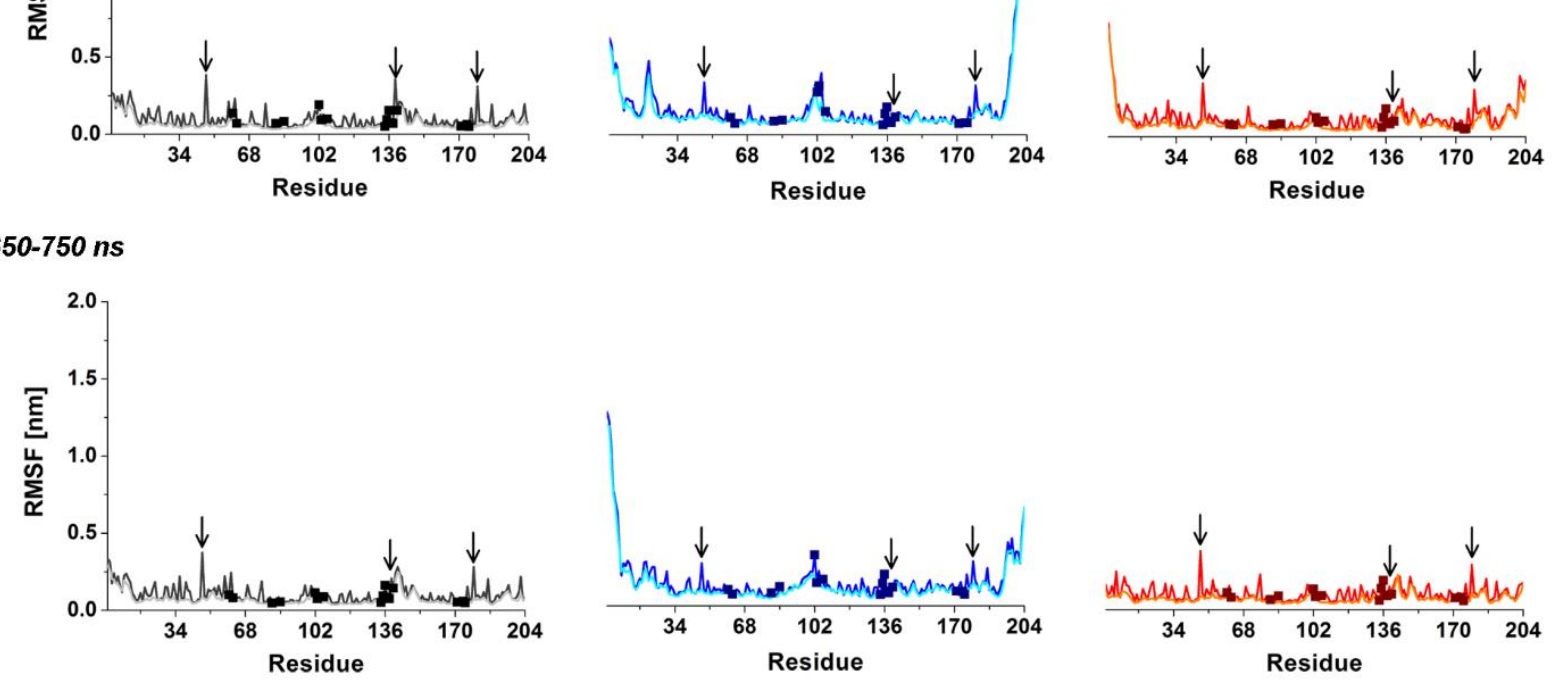

$900-1000$ ns
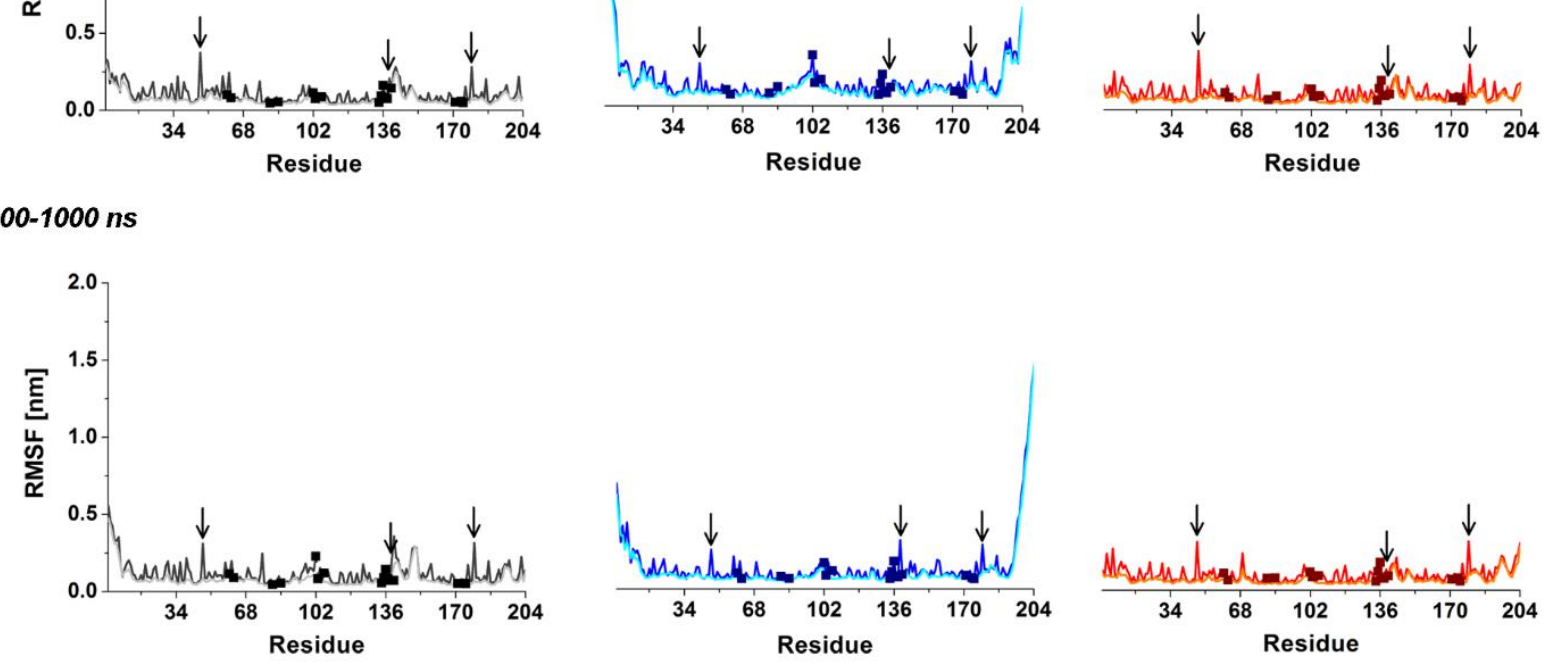

Figure S11. Root-mean-square fluctuations of the whole amino acid residues and of the $\mathrm{C}_{\alpha}$ atoms of the protein in the four trajectory segments of the NPT-iso, NPT-semi, and NP $\gamma$ T simulations; the amino acid residues from the binding pocket are denoted with squares; the N-glycosylated residues are marked with arrows 
The flexibility of the N-terminus of the protein in NPT-iso is evident from the peaks in the RMSF of the $\mathrm{C}_{\alpha}$ atoms of the first residues. Its behavior is similar throughout the simulation, but the mobility increases in the last TP. The position of the AAs is illustrated with the final snapshot in Figure 7 of the main manuscript. The N-terminus is located away from the protein and interacts with the membrane lipids. This is probably the reason for the deviations in the size and shape of the whole protein (Figures S9 and S10) and the proximity to the bilayer (Figure S3) at the end of the simulation. The C-terminus (connected to the GPI anchor) seems quite 'relaxed'. The positions of the N-glycosylated Asn (numbers 47, 139, and 179, colored in red in Figure 7 of the main manuscript) vary significantly, with the exception of Asn139 in the last two TPs. The lack of mobility for the latter residue can be linked to the elevated mobility of the backbone at Ser142 and Gly150 in this part of the trajectory. The AAs are located in the structured elements on both sides of Asn139 and their motions probably limit its propensity to move.

The different behavior of the receptor in NPT-semi is unmistakable. The fluctuations of the free $\mathrm{N}$-terminus (reaching ca. $0.5 \mathrm{~nm}$ ) are comparable in magnitude throughout the simulation to the final nanoseconds of the simulation in NPT-iso. In the 650-750 ns segment the N-terminus becomes even more mobile (RMSF up to $1.25 \mathrm{~nm}$ ), but this is only temporary, because in the final TP it returns to its previous state. However, the most distinctive feature of the protein in this trajectory is the C-terminus. The fluctuations are several times larger than the ones for the rest of FR $\alpha$, especially in the $450-550 \mathrm{~ns}$ part. An exception is the next time fragment, where they decrease in favor of mobility of the other protein end. During the last $100 \mathrm{~ns}$ the fluctuations rise again and in the final frame (Figure 7 of the main manuscript) we see that the C-terminus is seriously unfolded. The RMSF curves help link the structural variations in the two termini with the changes in the overall compactness, discussed in section Radius of gyration and ratios of moments of inertia of the main text. The backbone of several residues around Ser101 fluctuates more rapidly in the second and third TP. Some of them participate in the composition the binding pocket. The RMSF peak for His20 is elevated in 450-550 ns. These changes are not substantial in view of the global protein structure but more a consequence of the changes in compactness and shape. Unlike the other N-glycosylated residues, Asn139 is somewhat fixed in the first three parts of the trajectory, but 'recovers' its flexibility in the final TP. The backbone around residues Ser142 and Gly150 varies only in the 150-250 ns segment, but its movements fade in the rest of the trajectory. Hence, in 450-550 ns and 650-750 ns they are not linked to the restriction of the movements of Asn139.

The RMSFs of the N-terminus in NP $\gamma \mathrm{T}$ are higher than the ones of the remaining AAs in $150-200 \mathrm{~ns}(0.40 \mathrm{~nm})$. In the 450-550 ns fragment both termini experience particular fluctuations - up to $0.75 \mathrm{~nm}$ for the N-terminus and up to $0.40 \mathrm{~nm}$ for the C-terminus. This is in line with the evolution of the RMSD (Figure S2), the radius of gyration, and the ratios of the moments of inertia (section Radius of gyration and ratios of moments of inertia of the main manuscript). At the beginning of the time period the $\mathrm{N}$-terminus moves away from the protein, thus leading to changes 
in its global size and shape. Around $25 \mathrm{~ns}$ later the AAs in question come closer to the rest of the macromolecule. After $500 \mathrm{~ns}$ the $\mathrm{N}$-terminus is directed toward the membrane and interacts with the lipid heads (but not that much as in NPT-iso). The C-terminus changes its position to facilitate the protein orientation that allows such interactions. This, however, is reversible (confirmed also by visual observations) and the RMSFs of the termini are comparable to those of the protein core in the second half of the trajectory. As an exception, the fluctuations of the C-terminus in 900-1000 ns point out some other (minor) movement. It is attributed to bending of the protein towards the membrane but without participation of the other terminus. The residues from the binding pocket appear to be 'undisturbed'. In the 150-250 ns segment the peptide backbone around Val56 seems to be surprisingly mobile but this is only temporary. Asn47 and Asn179 have clear RMSF peaks throughout the trajectory and Asn139 is again immobilized. The residues around Ser142 and Gly150 have similar behavior to those in NPT-iso. Thus, the residue is again probably shielded by those AAs. The position of Asn179 in the last frame (Figure 7 of the main manuscript) is also rather interesting. It is located among the lipid heads but this apparently does not affect its flexibility. This orientation is realized due to the tilt of the protein in direction to the bilayer and it is discussed in detail in section Orientation of the receptor with respect to the membrane of the main text. Overall, the fluctuations of the amino acids are the smallest in NP $\gamma \mathrm{T}$. 
Table S7. Percentage of the secondary structure elements of the protein in the four trajectory segments of the NPT-iso, NPT-semi, and NP $\gamma \mathrm{T}$ simulations

\begin{tabular}{|c|c|c|c|c|c|c|c|c|c|}
\hline & Time [ns] & $\begin{array}{c}\text { Total } \\
\text { structured }^{\#}[\%]\end{array}$ & Coil [\%] & $\beta$-Sheet [\%] & $\beta$-Bridge [\%] & Bend [\%] & Turn [\%] & $\alpha$-Helix [\%] & 3-Helix [\%] \\
\hline \multicolumn{2}{|c|}{ X-ray } & 50 & 27 & 7 & 4 & 17 & 15 & 24 & 6 \\
\hline \multirow{4}{*}{ NPT-iso } & $150-250$ & $45 \pm 2$ & $30 \pm 1$ & $10 \pm 1$ & $3 \pm 1$ & $17 \pm 2$ & $15 \pm 2$ & $17 \pm 1$ & $5 \pm 1$ \\
\hline & $450-550$ & $44 \pm 3$ & $31 \pm 1$ & $9 \pm 2$ & $3 \pm 1$ & $17 \pm 2$ & $14 \pm 2$ & $18 \pm 1$ & $5 \pm 1$ \\
\hline & $650-750$ & $44 \pm 3$ & $32 \pm 2$ & $9 \pm 2$ & $3 \pm 1$ & $18 \pm 2$ & $14 \pm 2$ & $18 \pm 1$ & $6 \pm 2$ \\
\hline & $900-1000$ & $42 \pm 3$ & $29 \pm 2$ & $9 \pm 1$ & $2 \pm 1$ & $21 \pm 2$ & $13 \pm 2$ & $18 \pm 1$ & $6 \pm 2$ \\
\hline \multirow{4}{*}{ NPT-semi } & $150-250$ & $45 \pm 2$ & $29 \pm 2$ & $11 \pm 2$ & $2 \pm 1$ & $20 \pm 2$ & $13 \pm 3$ & $19 \pm 2$ & $6 \pm 2$ \\
\hline & $450-550$ & $46 \pm 2$ & $31 \pm 2$ & $11 \pm 2$ & $2 \pm 1$ & $19 \pm 2$ & $12 \pm 2$ & $21 \pm 1$ & $4 \pm 2$ \\
\hline & $650-750$ & $47 \pm 2$ & $29 \pm 2$ & $12 \pm 2$ & $2 \pm 1$ & $20 \pm 2$ & $12 \pm 2$ & $22 \pm 1$ & $4 \pm 1$ \\
\hline & $900-1000$ & $45 \pm 2$ & $30 \pm 2$ & $11 \pm 1$ & $2 \pm 1$ & $19 \pm 2$ & $11 \pm 2$ & $21 \pm 1$ & $5 \pm 1$ \\
\hline \multirow{4}{*}{$\mathbf{N P} \gamma \mathbf{T}$} & $150-250$ & $44 \pm 2$ & $32 \pm 2$ & $8 \pm 2$ & $3 \pm 1$ & $19 \pm 2$ & $12 \pm 2$ & $21 \pm 1$ & $6 \pm 2$ \\
\hline & $450-550$ & $45 \pm 3$ & $31 \pm 2$ & $9 \pm 2$ & $4 \pm 1$ & $19 \pm 2$ & $13 \pm 2$ & $20 \pm 2$ & $5 \pm 2$ \\
\hline & $650-750$ & $46 \pm 2$ & $31 \pm 1$ & $9 \pm 2$ & $4 \pm 1$ & $18 \pm 2$ & $13 \pm 2$ & $20 \pm 1$ & $5 \pm 2$ \\
\hline & $900-1000$ & $47 \pm 2$ & $30 \pm 1$ & $10 \pm 1$ & $4 \pm 1$ & $18 \pm 2$ & $14 \pm 2$ & $20 \pm 1$ & $5 \pm 2$ \\
\hline
\end{tabular}

\#Total structured: $\alpha$-Helix $+\beta$-Sheet $+\beta$-Bridge + Turn 
Secondary structure of FR $\alpha$
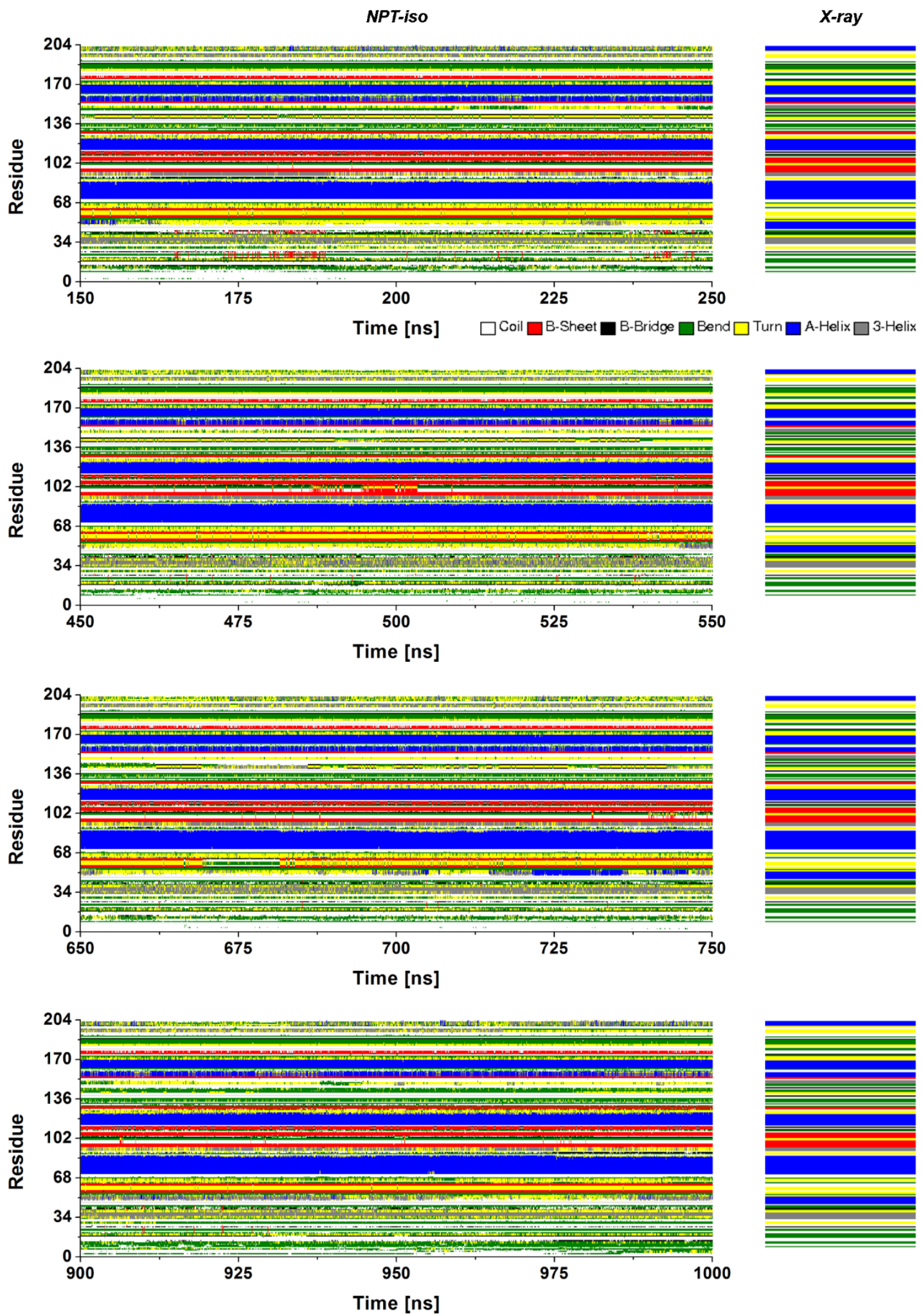

Figure S12. Evolution of the secondary structure of the folate receptor- $\alpha$ in the four trajectory segments of the NPT-iso simulation 

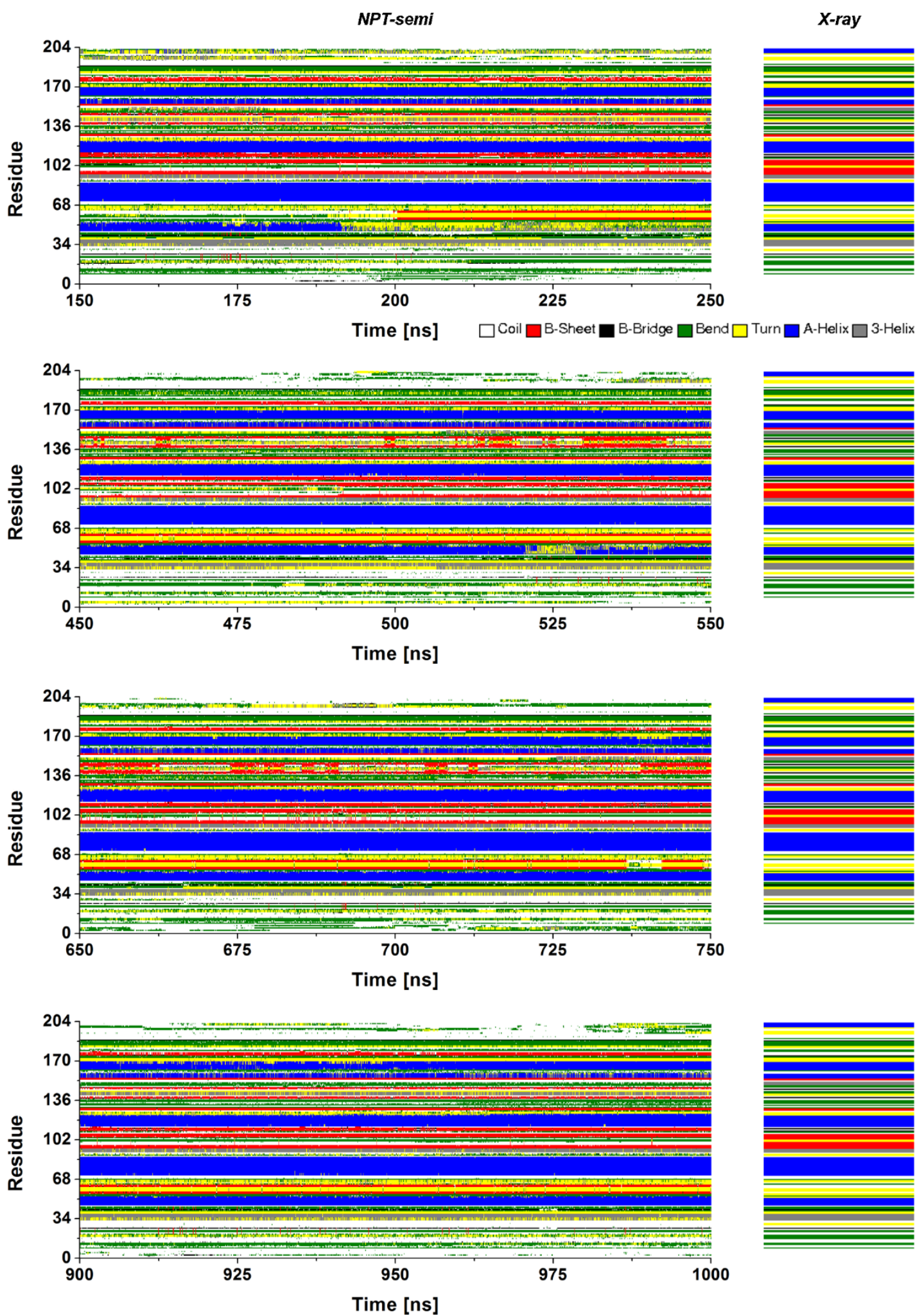

Figure S13. Evolution of the secondary structure of the folate receptor- $\alpha$ in the four trajectory segments of the NPT-semi simulation 

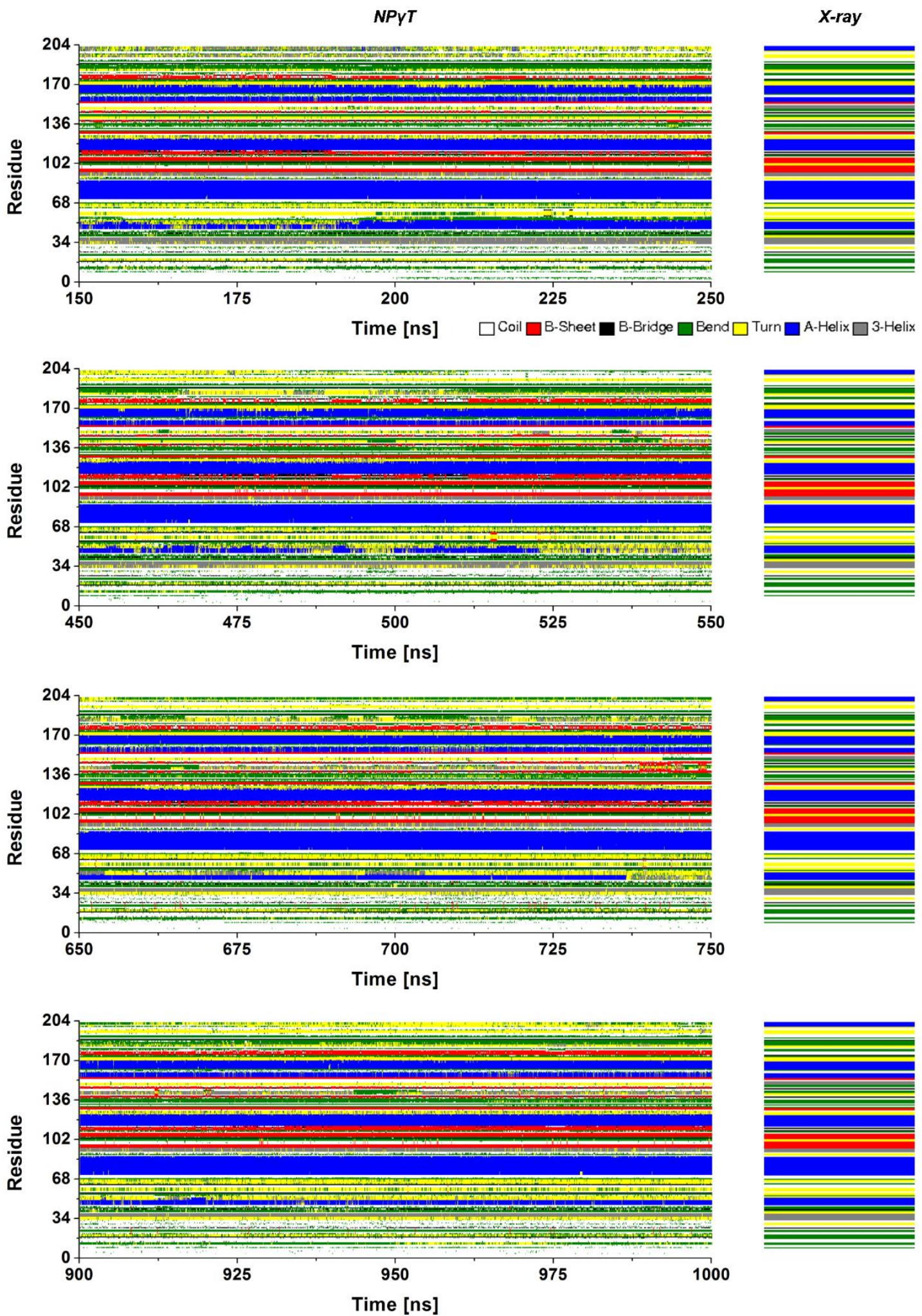

Figure S14. Evolution of the secondary structure of the folate receptor- $\alpha$ in the four trajectory segments of the NP $\gamma \mathrm{T}$ simulation 
The crystallographic structure of the receptor is characterized with six $\alpha$-helices, four $\beta$-strands (organized in two antiparallel sheets), and many loop regions (Figures S12-S14) [Ref. 40 of the main manuscript]. Starting from the N-terminus, the first $\alpha$-helix is completely unfolded in NPT-iso. It reappears briefly around $725 \mathrm{~ns}$ but disappears again at the end of the simulation. The helix is best retained in NPT-semi, although it vanishes in the second half of the 150-250 ns TP and for ca. $10 \mathrm{~ns}$ around $525 \mathrm{~ns}$. In NP $\gamma \mathrm{T}$ it is rather fragmentary throughout the first half of the trajectory, but is 'salvaged' in the second one, only smaller in size. The next four $\alpha$-helices are stable in all simulations, but the fifth one, located at the $\mathrm{C}$-terminus, disappears in all trajectories. This might be attributed to the interaction with the GPI and with the membrane lipids, since the C-terminus is the anchoring point of the protein. Regarding the four $\beta$-strands, they are all retained throughout the simulations, only the size of the two neighboring ones is decreased in favor of the connecting turn, which is restructured as a bend and a coil. New ones are formed - four in NPT-iso and NP $\gamma$ T and six in NPT-semi in equivalent parts of the sequence. So, together with the original ones, they form a total of four (NPT-iso and NP $\gamma$ T) and five (NPT-semi) double $\beta$-sheets. Two adjacent short $\beta$-strands separated by a turn are stably assembled by the same AAs in NPT-iso and NPT-semi, in the latter appearing after $200 \mathrm{~ns}$. This sheet is not present in the protein structure simulated in NP $\gamma \mathrm{T}$. In this trajectory we see only an 'attempt' by other residues to form such a construct. It appears also in NPT-semi where it is stabilized better. The strands even increase their size for short periods of time (not more than $10 \mathrm{~ns}$ ) in the second and third TP.

The behavior of the receptor termini allows clear distinction between the proteins in the three trajectories. The initial structuring of the $\mathrm{N}$-terminus is changed but to a different extent. Originally, it is composed of a continuous coil region, formed by the first eight residues, followed by alternating bends and coils. In NPT-iso, while preserved in the first three TPs, additional bends are introduced into the large coil in the final trajectory piece. The result of these changes is evident in Figure 7 of the main text. One can only speculate about a connection between the alterations in the SS of the protein terminus and its interaction with the lipids. In NPT-semi the changes are similar - there is additional structuring of the originally coiled part but it is rather transient in this simulation. It is most pronounced during the 650-750 ns time fragment, which explains the elevated RMSFs of this terminus (Figure S11). The terminus returns roughly to its initial state in the final $100 \mathrm{~ns}$ of the trajectory. The N-terminus in NP $\gamma \mathrm{T}$ retains its coil segment, except between 200-250 ns, when a short bend is formed. This is in line with the RMSF of the terminus in the first TP but not in the second one. Consequently, the motion of the Nterminus away from the protein and then (with the whole macromolecule) toward the membrane does not affect its secondary structure in NP $\gamma \mathrm{T}$.

In the initial geometry of the protein the GPI-bound C-terminus is assembles as an $\alpha$-helix. In NPT-iso this element is replaced by interchanging turn and 3-helix. The neighboring AAs are analogously perturbed. This is the case also in the 150-250 ns piece of the NPT-semi trajectory, only the formation of the 3-helix is less frequent. Then, a major destructuring is observed, which corresponds to the significant fluctuations (Figure S11). In the next TP, a stable construct of interconverting bend and turn is formed, which appears to stabilize the terminus, thus reducing the RMSF. Evidently, the Ctail unfolds again in the final part until it structures back in the final ca. 15 ns (Figure 7 of the main 
manuscript). In the first trajectory segment of the NP $\gamma \mathrm{T}$ simulation, the terminal residues are similarly structured to those in NPT-iso. Then, turn elements become predominant, followed by uncoiling after $510 \mathrm{~ns}$. It is probably connected to the discussed bending toward the membrane (see section Rootmean-square fluctuations of the amino acids). After that the C-terminus is stably structured.

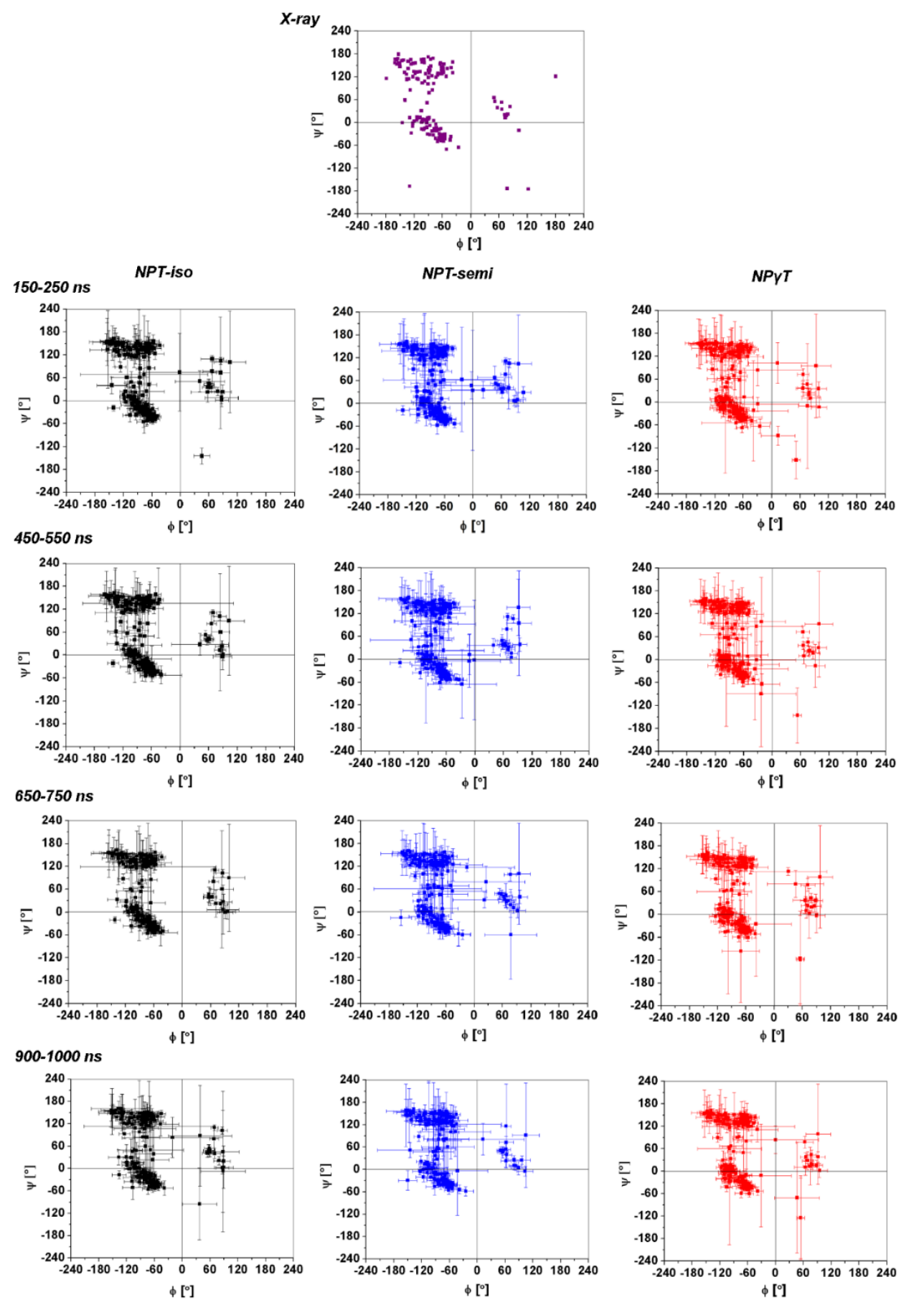

Figure S15. Ramachandran plots of the folate receptor- $\alpha$ in the crystallographic structure of its complex with folate (top) and in the four trajectory segments (from second row to bottom) of the NPT-iso (left), NPT-semi (middle), and NP $\gamma$ T (right) simulations 
Table S8. Average volume (with standard deviations) of the binding pocket of the folate receptor$\alpha$ in the four trajectory segments of the NPT-iso, NPT-semi, and NP $\gamma$ T simulations

\begin{tabular}{|c|c|c|c|}
\hline & \multicolumn{3}{|c|}{ Pocket volume $\left[\mathbf{n m}^{\mathbf{3}}\right]$} \\
\hline Time [ns] & NPT-iso & NPT-semi & NP $\boldsymbol{\gamma} \mathbf{T}$ \\
\hline $\mathbf{1 5 0 - 2 5 0}$ & $0.380 \pm 0.083$ & $0.363 \pm 0.099$ & $0.353 \pm 0.076$ \\
\hline $\mathbf{4 5 0 - 5 5 0}$ & $0.359 \pm 0.057$ & $0.393 \pm 0.091$ & $0.275 \pm 0.070$ \\
\hline $\mathbf{6 5 0 - 7 5 0}$ & $0.342 \pm 0.079$ & $0.408 \pm 0.097$ & $0.278 \pm 0.058$ \\
\hline $\mathbf{9 0 0 - 1 0 0 0}$ & $0.308 \pm 0.060$ & $0.370 \pm 0.098$ & $0.296 \pm 0.052$ \\
\hline
\end{tabular}

$X$-ray: $0.326 \mathrm{~nm}^{3}$ 

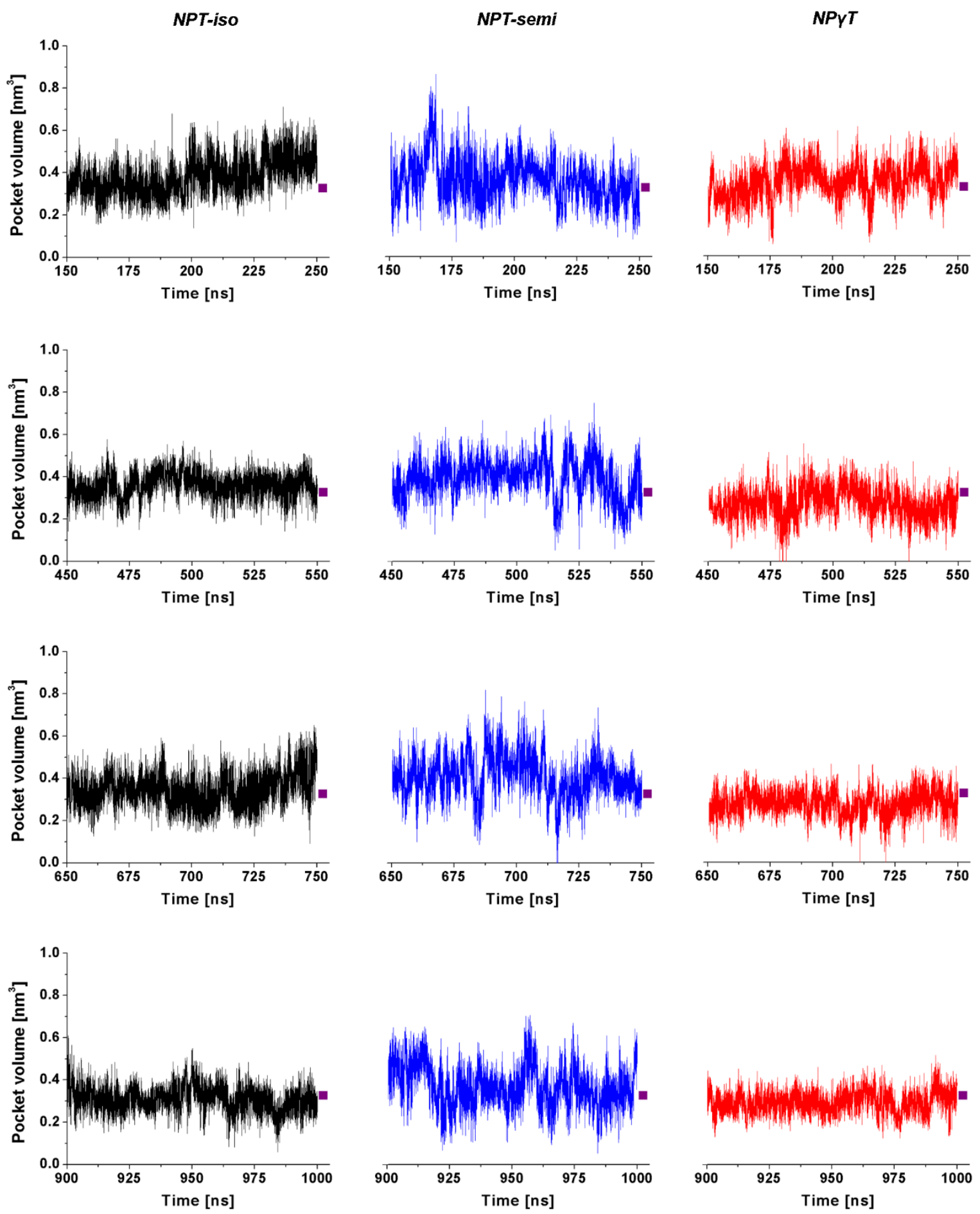

Figure S16. Time dependence of the volume of the binding pocket of the folate receptor- $\alpha$ in the four trajectory segments (from top to bottom) of the NPT-iso (left), NPT-semi (middle), and NP $\gamma \mathrm{T}$ (right) simulations; the value for the experimental structure of $\mathrm{FR} \alpha$ is depicted with a purple square 

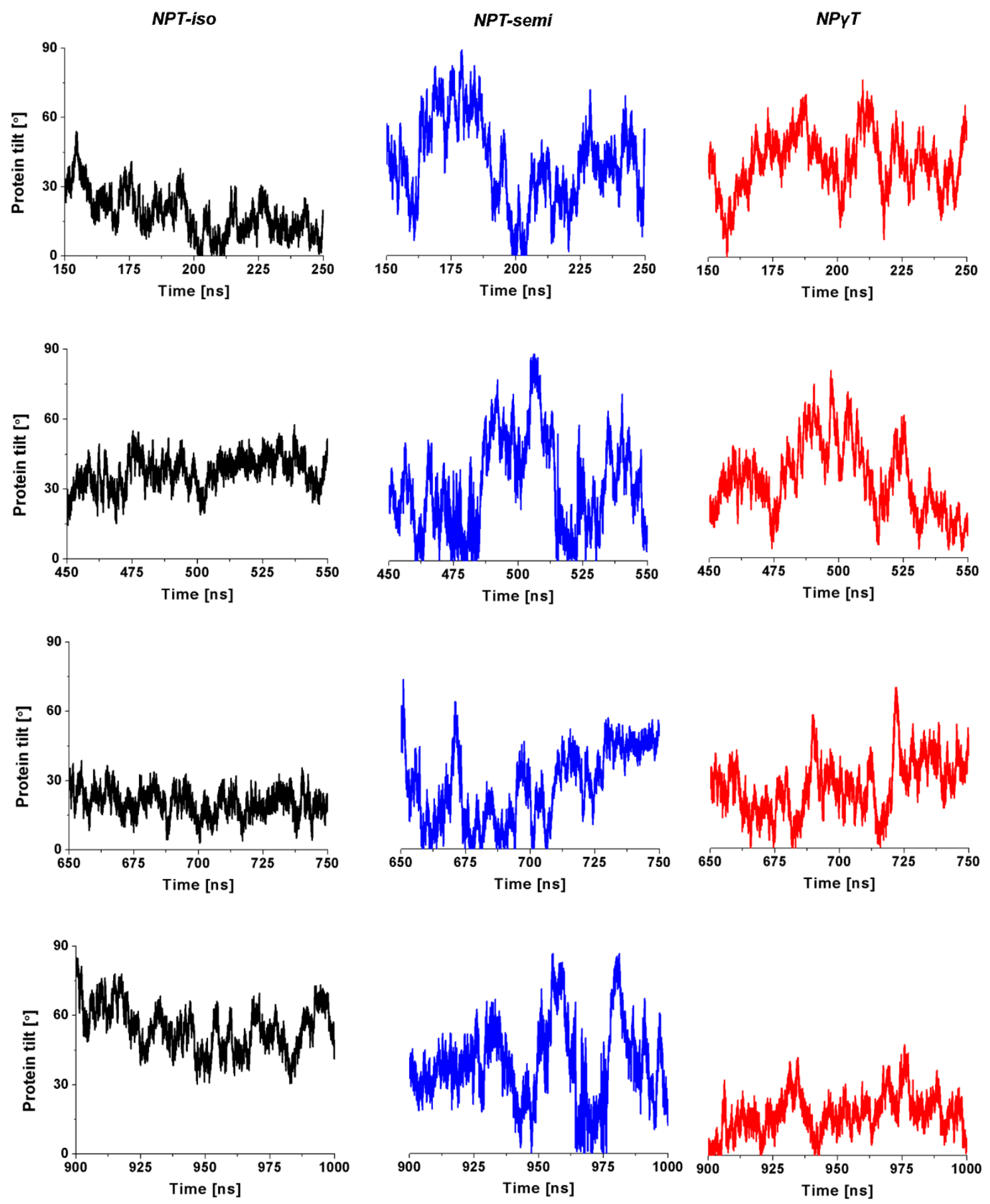

Figure S17. Time dependence of the tilt of FR $\alpha$ relative to the membrane plane in the four trajectory segments (from top to bottom) of the NPT-iso (left), NPT-semi (middle), and NP $\gamma \mathrm{T}$ (right) simulations 

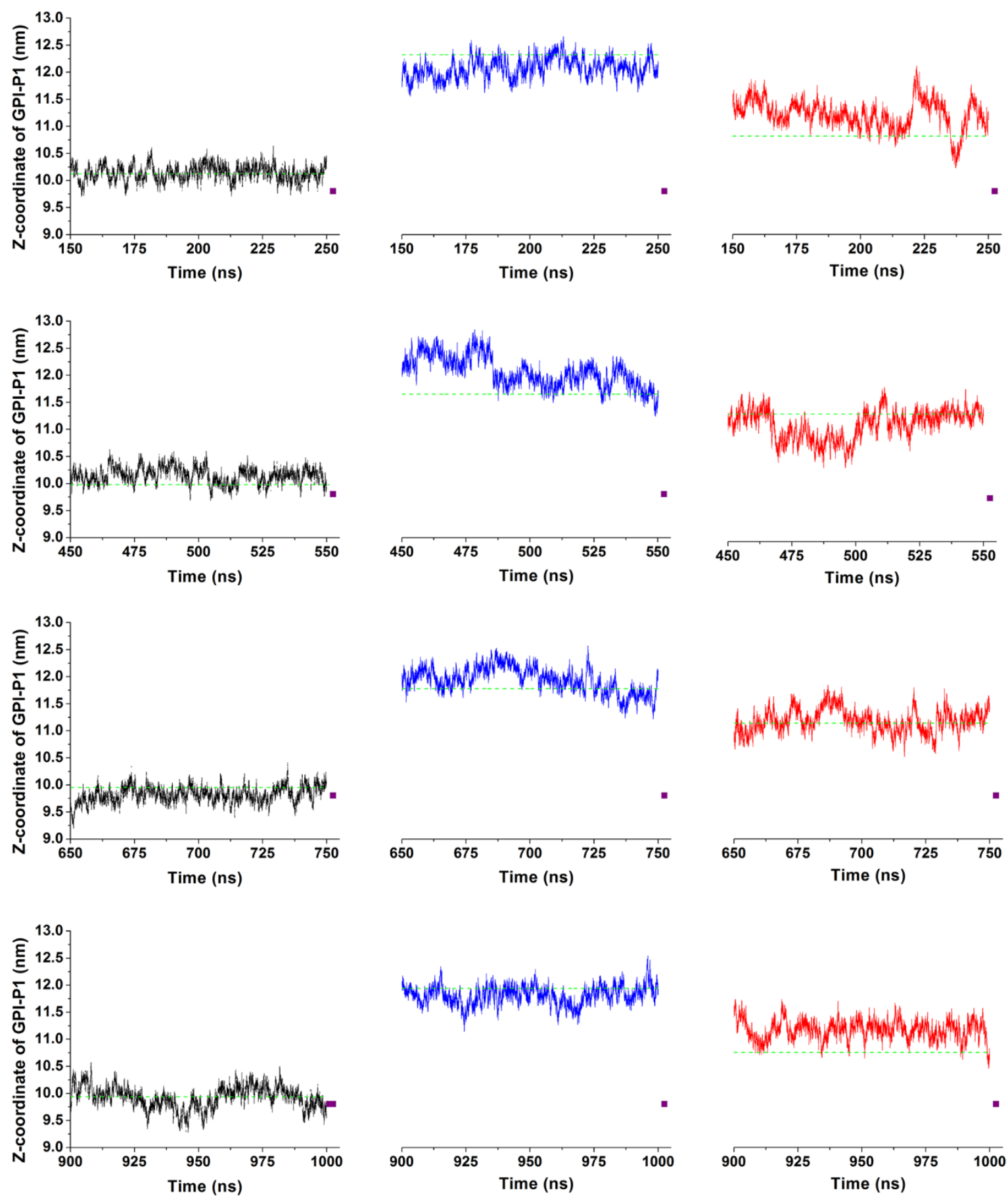

Figure S18. Evolution of the z-coordinate of the atom P1 (see Figure S1 for notation) of the GPI anchor in the four trajectory segments (from top to bottom) of the NPT-iso (left), NPT-semi (middle), and NP $\gamma \mathrm{T}$ (right) simulations; the value for the initial structure is depicted with a purple square and the equimolecular dividing surface is shown as a dashed green line 

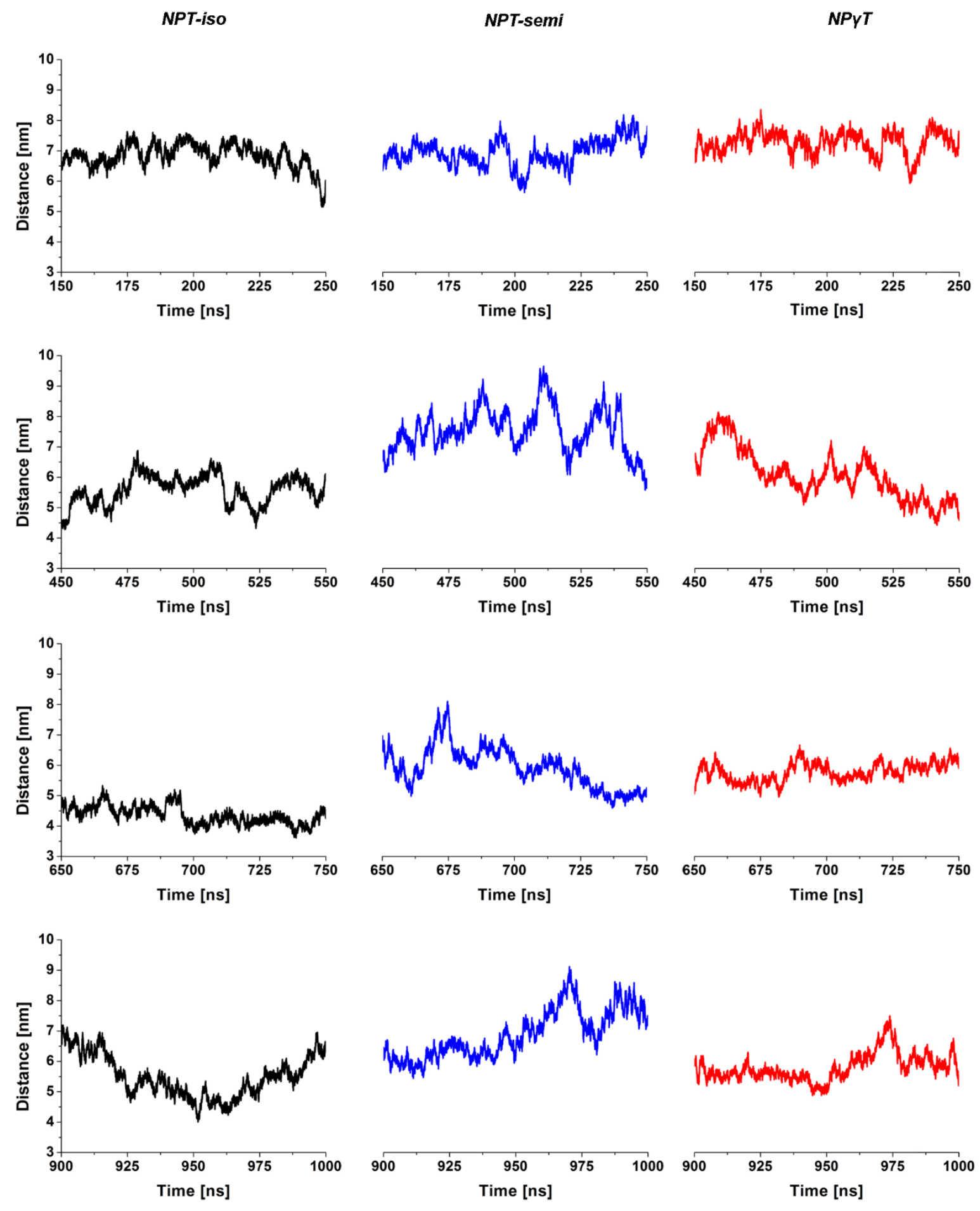

Figure S19. Time dependence of the distance from the COM of the FR $\alpha$ binding pocket to the COM of the lipid bilayer in the four trajectory segments (from top to bottom) of the NPT-iso (left), NPT-semi (middle), and NP $\gamma \mathrm{T}$ (right) simulations 

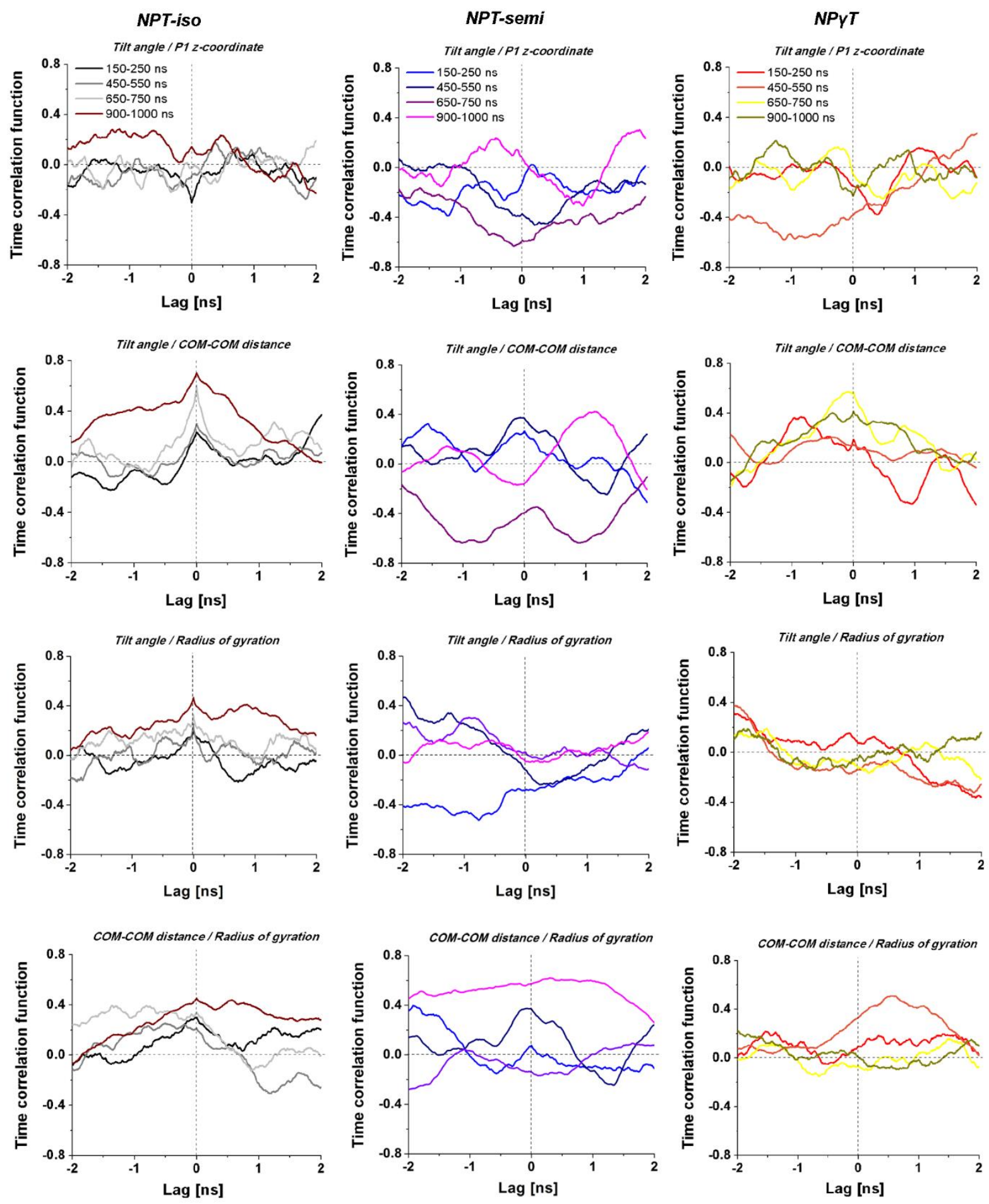

Figure S20. Time correlation functions of the protein tilt angle with the z-coordinate of P1 from GPI, with the COM-COM distance protein pocket - membrane, and with the radius of gyration of the protein and of the COM-COM distance with the radius of gyration in the four trajectory segments (from top to bottom) of the NPT-iso (left), NPT-semi (middle), and NP $\gamma \mathrm{T}$ (right) simulations 

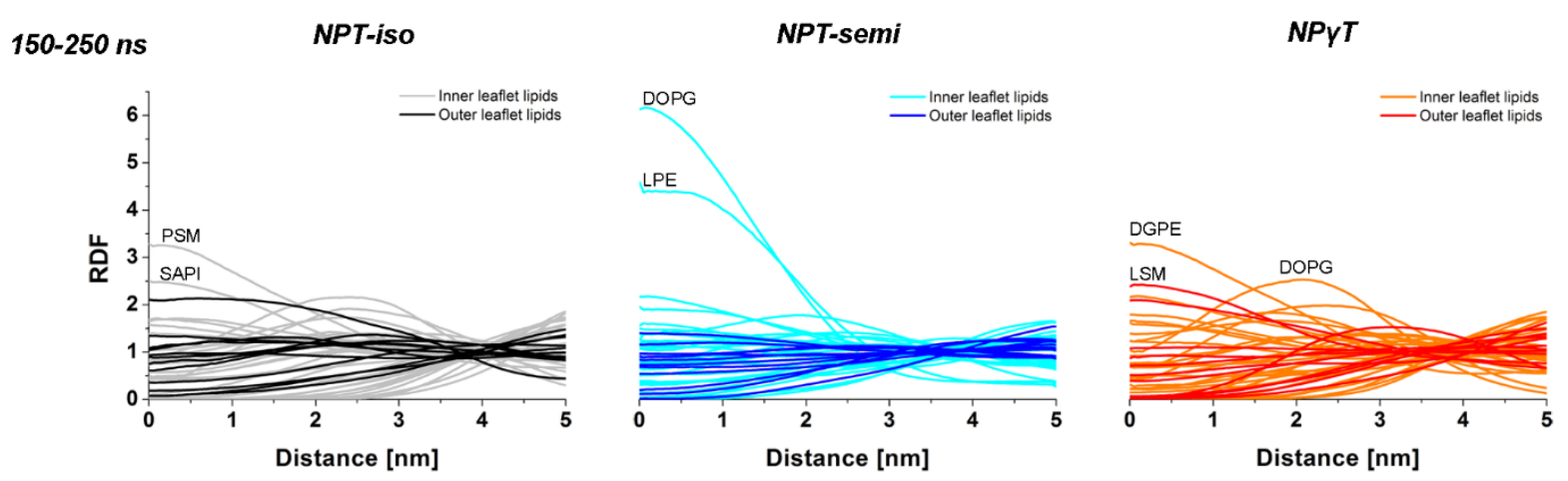

450-550 ns
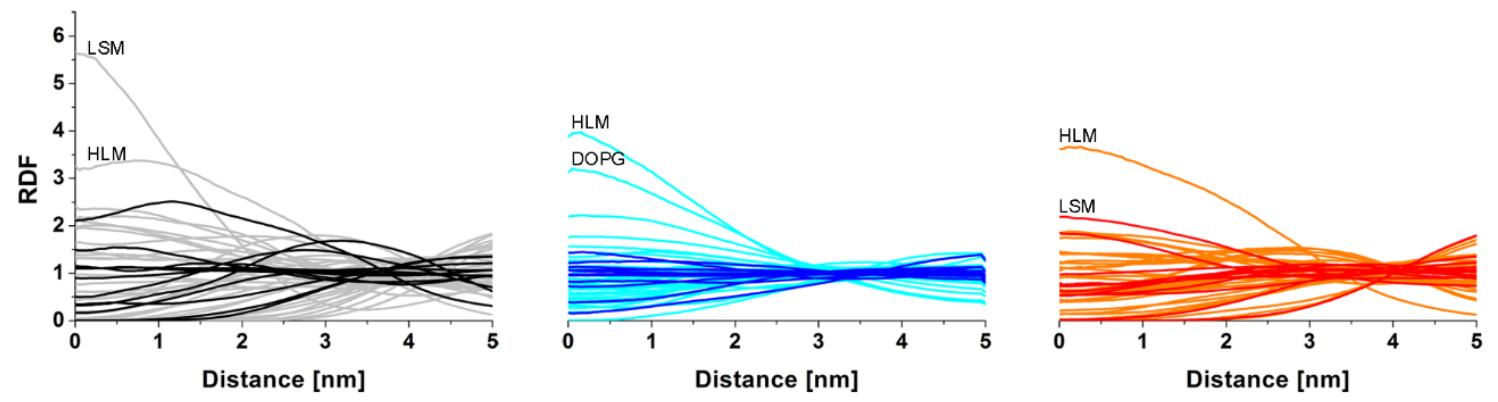

$650-750$ ns

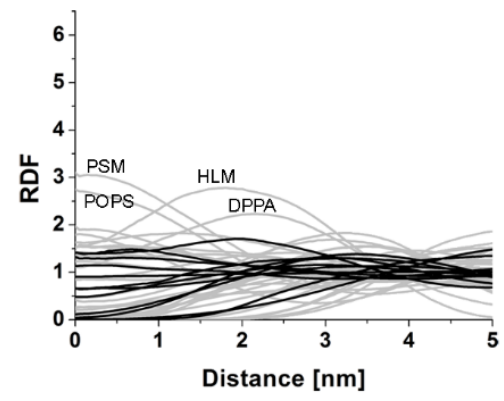

900-1000 ns
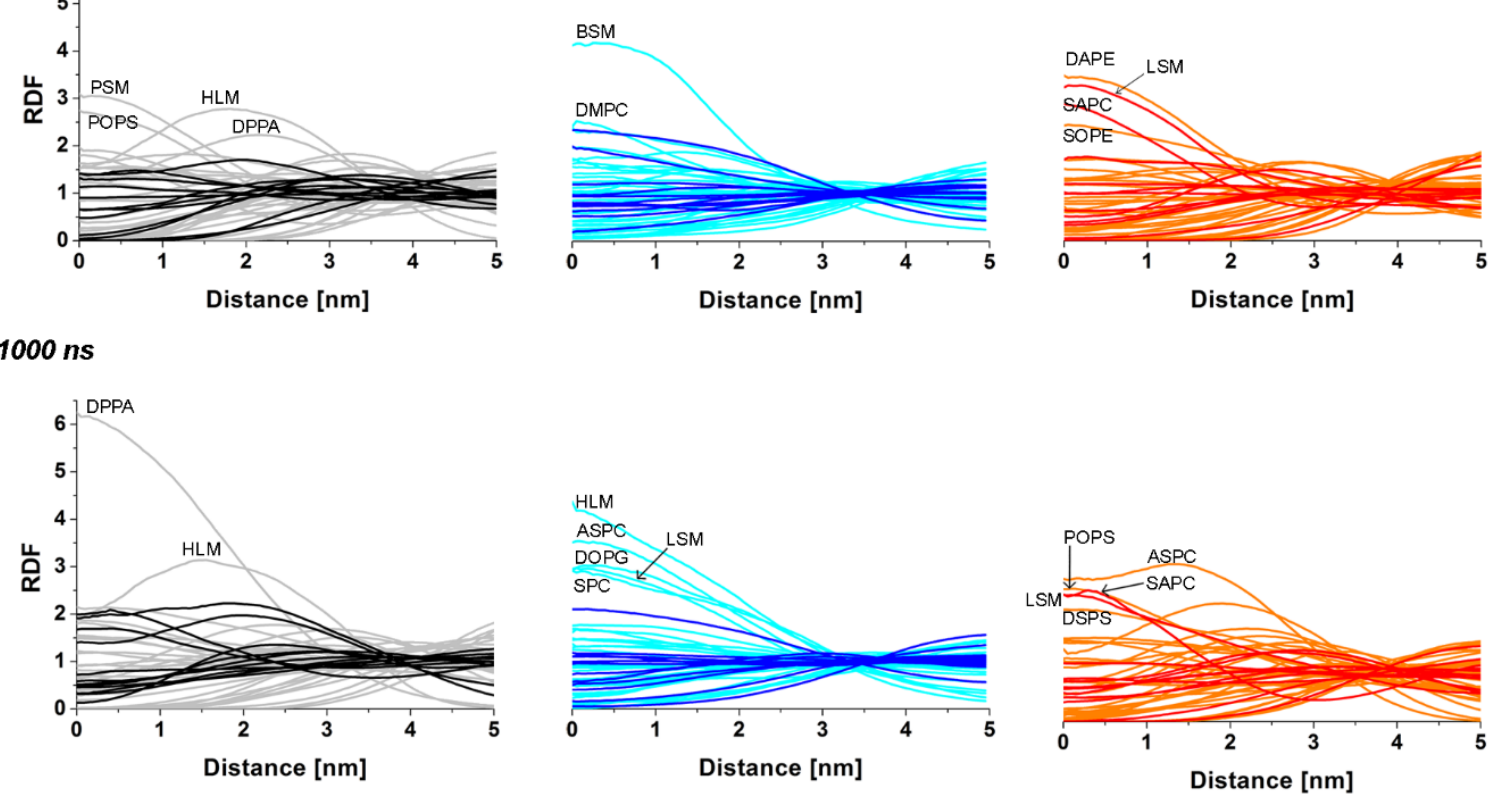

Figure S21. 2D radial distribution functions of the distance from the COM of FR $\alpha$ to the phosphorous atom of the phospholipids or the base carbon atom of CHL in the four trajectory segments (from top to bottom) of the NPT-iso (left), NPT-semi (middle), and NPrT (right) simulations; the data are averaged over all molecules from a given lipid type in the particular leaflet 